\title{
Big Data, Data Analytics und Smart Services rund um Wohnen, Gesundheit und Mobilität: Bürgerschreck und Hoffnungsträger in privaten Lebenswelten
}

\subsection{Grundlagen zum Konzept der Lebenswelten}

\subsubsection{Begriff der Lebenswelten}

Jeder Mensch befindet sich und handelt in verschiedenen Lebenswelten. Eine erste einfache Definition gibt der Duden und beschreibt eine Lebenswelt als „,persönliches Umfeld; Welt, in der sich jemandes Leben abspielt“ (Duden 2019). Seine Wurzeln hat der Begriff ,Lebenswelt" in der philosophischen Strömung der Phänomenologie und er wurde insbesondere durch den Philosophen Edmund Husserl geprägt. Nach seiner Definition umfassen Lebenswelten den menschlichen Wirklichkeitsbereich und dessen egologische Beziehung zur Umwelt. Es ist das Erleben einer Welt, die von Anderen mit vergleichbarer Haltung und Erfahrungsschatz gleichartig empfunden wird (Spektrum 2001). Eine Lebenswelt erklärt das Umfeld, das ein Subjekt (oder eine Gruppe) im Alltag wahrnimmt und in dem es sich bewegt. Diese Wahrnehmung wird zum Bestandteil seiner subjektiven Lebenswelt (Mamerow 2012, S. 7).

Die Subjektivität führt dazu, dass Lebenswelten von jedem Menschen anders wahrgenommen werden und daher nicht objektiv abgegrenzt werden können. Eine Lebenswelt kann für eine einzelne Person über eine Spannbreite bestimmt sein, die sich von übergeordneten Themen (wie Wohnen, Gesundheit und Mobilität) bis hin zu kleinsten Erlebnisbereichen erstrecken kann. Auf der Definition von Husserl aufbauend, beschreiben Schütz und Luckmann die Lebenswelt als „Wirklichkeit, an der der Mensch in unausweichlicher, regelmäßiger Wiederkehr teilnimmt“" (Schütz/Luckmann 2017, S. 29). Die Verwendung des Ideenansatzes

Dieses Kapitel wurde von Fred Wagner und Theresa Jost verfasst.

(C) Der/die Herausgeber bzw. der/die Autor(en) 2020 
der Lebenswelten eröffnet in vielerlei Hinsicht Chancen. Theoriekonzepte sowie praktische Vorgehensweisen, die auf Lebenswelten basieren, haben den Vorteil, ganzheitlich angelegt zu sein.

Lebenswelten berühren aus der Sicht von Bürgern alle möglichen Themenfelder des alltäglichen Lebens und unterscheiden sich in ihrer Komplexität und Tiefe. Allgemeiner berühren Lebenswelten den Handlungsraum, das Umfeld oder die Denkweise, in denen sich Bürger täglich bewegen. Sie lassen sich nach ihrer Identität, ihrer Struktur und ihrem räumlichen Bezug unterscheiden. Meist werden Lebenswelten im privaten und geschäftlichen Kontext differenziert. Jedoch überschneiden sich Lebenswelten oft und können, je nach Situation oder Perspektive, ineinander übergehen. Daher haben Lebenswelten eine gewisse Unschärfe. Viele Lebenswelten von Bürgern können einer bestimmten Klientel zugeordnet werden, spiegeln eine bestimmte Orientierung wider und können Aufschluss über, die Lebensweise" geben. Damit geben Lebenswelten teilweise auch Einsichten in die physische und psychische Kondition eines Bürgers (Kraus 2006). Auch daraus ergibt sich, dass Lebenswelten nicht konstant sind, sondern sich im Verlauf des Lebens je nach aktueller Lebenssituation und Umweltfaktoren dynamisch verändern. Ein wichtiger Einflussfaktor sind dabei gesellschaftspolitische Entwicklungen und der technologische Fortschritt, der sich auf die Lebenswelten auswirkt, in denen Bevölkerungsgruppen zu Hause sind.

\subsubsection{Tatsächliche Lebenswelten aus Sicht von Bürgern}

Lebenswelten können von großen Themenkomplexen geprägt, aber auch sehr fein untergliedert sein. Eine objektive und pauschale Kategorisierung ist nicht möglich, vielmehr sind subjektive und standpunktabhängige Kriterien hinzuzuziehen. Zudem weichen die Auffassungen über die Relevanz und Abgrenzung bestimmter Lebenswelten in verschiedenen Ländern stark voneinander ab. Aus Sicht von Bürgern ist damit die Einteilung der Lebenswelten nicht immer trennscharf und für alle Milieus gleichermaßen umsetzbar, da je nach Kontext, sozio-ökonomischen und kulturellen Unterschieden Lebenswelten sehr differenziert wahrgenommen werden. Es ist allerdings zweckmäßig, den Lebenswelten einen geeigneten Rahmen zu geben und zentrale Lebenswelten voneinander abzugrenzen, um in theoretischen Rahmenbedingungen arbeiten zu können, aber auch um in der Praxis gesamtheitlich nach Lösungen bestimmter Probleme suchen zu können. Generell stellt sich die Frage, ob sich für die Einordnung von Lebenswelten ein allgemeingültiges Schema aufstellen lässt oder diese nur in einem bestimmten Kontext zu sehen sind, in dem sie interpretiert werden können. Dies wird unterschiedlich 
gehandhabt. Beispielsweise unterteilt die Gesellschaft für Konsum-, Markt- und Absatzforschung e. V. die für die Marktforschung relevanten Lebenswelten zum einen in eine biografische, um die persönliche Lebensumwelt einer Person zu erfassen, und zum anderen in eine familiäre Lebenswelt, um auch private Haushalte einzuschließen. Lebenswelten lassen sich dann feiner nach Erwerbssituation, kulturellem Kontext, demografischen Faktoren (insbesondere Alter), monetären oder sozialen Rahmenbedingungen sowie familiären Strukturen und Verhaltensweisen differenzieren (Corthier et al. 2011, S. 5).

Für die vorliegende Arbeit wurde den Lebenswelten ebenfalls ein vorgegebener Rahmen gesetzt. Der Fokus lag dabei allerdings weniger auf persönlichen, individuellen Umständen, unter denen die Menschen leben. Stattdessen wurde schwerpunktmäßig auf Lebensbereiche abgestellt, in denen sich die Menschen bewegen, mit denen sie sich beschäftigen und die sie für sich gestalten wollen. Das ist z. B. die Wohnung, in der sich der Bürger privat aufhält und zu Hause fühlt, und die mit der, Lebenswelt Wohnen ' abgedeckt wird. Ein zentrales Thema für die meisten Menschen ist das körperliche, geistige und seelische Wohlbefinden, mit dem die ,Lebenswelt Gesundheit " angesprochen ist. Die Möglichkeiten, sich räumlich von A nach B zu bewegen, führen die ,Lebenswelt Mobilität ‘ zusammen. Interessen und Aktivitäten im beruflichen Umfeld bilden die Lebenswelt Arbeit, das Zusammenleben in der häuslichen Gemeinschaft die Lebenswelt Familie usw. Zentrale Lebenswelten, die für gewöhnlich hohe Relevanz für die meisten Menschen besitzen, sind ohne Anspruch auf Vollständigkeit, trennscharfe Strukturierung und Priorisierung (da diese letztlich subjektiv geprägt, s. o.) z. B. folgende:

1. Wohnen

2. Gesundheit

3. Mobilität

4. Arbeit

5. Familie

6. Freizeit

7. Sport

8. Urlaub

9. Kommunikation

10. Recht

11. Finanzen

12. Altersvorsorge 
Innerhalb einer Lebenswelt kann weiter differenziert werden. In Freizeit und Sport (die sich vielfach überlappen) können etwa die Lebenswelten des Fußballs und des Motorrads unterschieden werden. Unter den Motorradfahrern grenzen sich viele Harley Davidson-Fahrer vermutlich mit dem Empfinden einer eigenen Lebenswelt ab. Zugleich verschwimmen Lebenswelten, wenn z. B. Arbeit und Freizeit räumlich und zeitlich nicht mehr strikt getrennt werden. Die Orientierung, in der eine Lebenswelt wahrgenommen wird, ist also ebenfalls ein wichtiger Faktor, um die richtigen Schlüsse zu ziehen.

Aus den übergeordneten Lebenswelten stechen Wohnen, Gesundheit und Mobilität aus dem Grund besonders hervor, weil der Bürger mit ihnen permanent konfrontiert wird. Zudem betreffen diese Lebenswelten nicht nur einzelne Gruppen, sondern jeder Bürger ist von ihnen betroffen. Darüber hinaus stehen die Lebenswelten Wohnen und Gesundheit für sehr persönliche Themen und müssen daher - gerade im Hinblick auf die Nutzung von Daten - besonders sensibel gehandhabt werden. Auch die Mobilität ist sensibel, soweit aus entsprechenden Daten Bewegungsprofile abgeleitet werden können. In der vorliegenden Arbeit, die das Spannungsfeld zwischen ,Datennutzung ' und ,Datenschutz' beleuchtet, werden daher Smart Services und verschiedene Geschäftsmodelle in gerade diesen drei Lebenswelten exemplarisch analysiert.

Da es in den drei Lebenswelten Wohnen, Gesundheit und Mobilität Überschneidungen gibt, sind nicht alle für eine Lebenswelt vorgenommenen Analysen trennscharf zu denen einer anderen Lebenswelt. So sind beispielsweise smarte Assistenz-systeme für immobile Personen sowohl der Lebenswelt Wohnen als auch Gesundheit zuzuordnen.

\subsubsection{Big Data und Data Analytics in den Lebenswelten}

\subsubsection{Big Data}

,Big Data' steht für die Verfügbarkeit großer digitaler Datenmengen und deren technologische Auswertungsmöglichkeiten. Der Trend zu ,Big Data ' wird durch die zunehmenden Speicherkapazitäten und die steigende Verarbeitungsgeschwindigkeit neuer Computertechnologien getrieben und beschleunigt. Zur Charakterisierung von ,Big Data können vier Dimensionen von Kriterien herangezogen werden: Volume, Velocity, Variety und Value (IBM 2019). 
Volume beschreibt ,Big Data' mit der verfügbaren Datenmenge und der Maßgröße ,Byte‘. Die jährlich generierte Datenmenge wird vor allem in den nächsten Jahren rasant wachsen. Wurden 2016 16,1 ZetaByte $^{1}$ an digitalen Daten weltweit generiert, soll sich diese Zahl bis zum Jahr 2025 verzehnfachen (siehe hierzu Statista 2019). Das Kriterium Velocity steht für die Geschwindigkeit der Datenerzeugung und Verarbeitung.

Die Datenvielfalt ist Gegenstand des Kriteriums Variety. Damit sind nicht lediglich unterschiedliche Dateiformate (z. B. Bilder, E-Mails, PDF, WordDateien, Videos etc.) gemeint, sondern auch ihr Strukturierungsgrad. Daten sind unstrukturiert, wenn sie keiner formalisierten Ordnung entsprechen. Dies ist i. d. R. bei Bildern und E-Mails der Fall. Semistrukturierte Daten weisen entweder keine fest typisierte, sondern lediglich eine versteckte Struktur auf oder sind insgesamt unterschiedlich strukturiert. Mit der Struktur sind neben den Dateiformaten, die mit der Unternehmensdatenbank kompatibel sein müssen, auch die Strukturen des Feldtypen gemeint, die eine Datei in einer Datenbank näher beschreibt (Deutsches Institut für Vertrauen und Sicherheit im Internet 2016, S. 26). Eine weitere Einteilung differenziert in statische und dynamische Daten. Statische Daten werden einmal erfasst und liegen dann unverändert vor.

Dagegen lassen sich die Merkmale von dynamischen Daten verändern und neue Informationen hinzufügen (Denkena et al. 2017, S. 299). Nach den Bezugsgrößen können Personendaten, Objektdaten und Prozessdaten unterteilt werden. ${ }^{2}$ Je nach Datentyp lassen sich Datenanalysen zur Generierung mehrwertiger Informationen mehr oder weniger effektiv und effizient umsetzen (Sobe 2018, S. 6). Die Investitionen von Unternehmen in Informationstechnologien zur Speicherung und Verarbeitung von ,Big Data' sollen sich selbst-verständlich amortisieren und den Unternehmenswert steigern. Anders ist die Sammlung, Speicherung und Auswertung von ,Big Data' aus unternehmerischen Gesichtspunkten nicht sinnvoll. Diese Zielsetzung ist im Kriterium Value enthalten (Fasel/Maier 2016, S. 6).

Für die folgende Arbeit sind insbesondere maschinelle Daten relevant, die digitale Informationen enthalten. Erzeugt werden die Maschinendaten von immobilen oder mobilen und möglichst vernetzten Endgeräten, Computern und eingebetteten Systemen (Roussem 2017).

\footnotetext{
${ }^{1} 1$ Zetabyte entspricht ca. $10^{21}$ Byte.

${ }^{2}$ Eine ähnliche Unterteilung, die allerdings Schnittmengen aufweist, differenziert Personendaten, Haushaltsdaten, Gebäudedaten, Objektdaten, Gewerbedaten und Device-Daten (Beenken et al. 2018).
} 


\subsubsection{Data Analytics in den Lebenswelten}

Data Analytics bezeichnet Verfahren, bei denen Daten aus verschiedenen Datenquellen extrahiert und auf bestimmte Fragestellungen hin untersucht werden. Dabei sollen Schlussfolgerungen gezogen werden, die in einem bestimmten Zusammenhang zu den analysierten Daten stehen. Häufig taucht Data Analytics im Zusammenhang mit Data Mining auf. Beim Data Mining geht es darum, neue und noch unbekannte Zusammenhänge aus Daten herzustellen (Bardmann 2019, S. 579).

Durch die Datenerhebung und -auswertung können und sollen Mehrwerte für die Bürger in ihren Lebenswelten entstehen. Dafür werden im Rahmen der Datenanalyse zunächst Einstellungen, Verhaltensweisen und sonstige Merkmale der Bürger erfasst, um daraus ihre Bedürfnisse abzuleiten. Auf Basis der Erkenntnisse sollen Angebote ermöglicht werden, mit denen diese Bedürfnisse erfüllbar sind - und dies immer individueller. Zudem soll mithilfe von Data Analytics die Zukunft vorhersehbarer werden. Beispielsweise können zugunsten der ,Lebenswelt Gesundheit" nicht nur bereits eingetretenen Krankheiten frühzeitig erkannt und schneller und damit erfolgreicher therapiert werden, sondern es eröffnen sich auch Möglichkeiten, drohende Krankheiten aufzudecken, um sie mit präventiven Maßnahmen noch abzuwenden. ${ }^{3}$

Generell bieten Data Analytics auch in der Forschung und Entwicklung über nahezu alle Wissenschaftsdisziplinen hinweg Potenziale zur Verbesserung der Lebensqualität. Big Data und deren Analyse haben damit die Wirkungsfähigkeit, die Lebenssituation jedes einzelnen Bürgers zu verbessern. Allerdings sind gleichzeitig die Risiken, vor allem durch fehlende Datensicherheit, nicht zu unterschätzen. Besonders die ausdrücklich vom Bürger nicht gewollte Verwendung von persönlichen Daten durch Dritte muss reguliert werden. So ist möglichst zu verhindern, dass Daten ohne Einverständnis der betroffenen Personen erfasst, individuell ausgewertet und für Eigeninteressen von Unternehmen oder beispielsweise zur politischen Beeinflussung der Bürger verwendet werden.

\footnotetext{
${ }^{3}$ Mithilfe der Apple Watch und damit erhobener Vitaldaten ihrer Träger ist es amerikanischen Forschern bereits gelungen, unter Nutzung von Data Analytics und Backtesting-Verfahren typische Muster zu erkennen, die Patienten mit Herzvorhof-Flimmern bereits vor Eintritt der Krankheit aufwiesen. Umgekehrt soll es damit schon heute möglich sein, mit den entsprechenden Mustererkennungen von Echtzeitdaten mit einer Treffsicherheit von über $95 \%$ vorherzusagen, dass ein bestimmter Träger der Apple-Watch unter sonst gleichbleibenden Umständen in Zukunft Herzvorhof-Flimmern erleiden wird. Mit geeigneten Präventivmaßnahmen kann dies aber häufig noch verhindert werden.
} 
Um möglichst viele Chancen für mehrwertige Erkenntnisse zu schaffen, ist es hilfreich, Daten unbegrenzt sammeln und auswerten zu können. Oft ergeben sich die Anwendungsfelder erst nach Mustererkennungen in Big Data, die mithilfe von Data Analytics hervorgebracht wurden und an die zuvor überhaupt nicht gedacht worden war. Eine getrennte Erhebung der Daten, beispielsweise nach Lebenswelten, ist von daher gar nicht zweckmäßig. Die größten Chancen bieten Datenpools über die Grenzen der verschiedenen Datentypen und Lebenswelten hinweg. Allerdings sieht der Datenschutz - verständlicherweise - gerade diese Form der Datenerhebung und Datenauswertung als besonders problematisch an. Insbesondere die EU-DSGVO mit den Erfordernissen der Datenminimierung ${ }^{4}$ gem. Art. 5 Abs. 1 c EU-DSGVO, Speicherbegrenzung ${ }^{5}$ gem. Art. 5 Abs. 1 e EU-DSGVO und Zweckbindung $^{6}$ gem. Art. 5 Abs. 1 b EU-DSGVO beschränken die faktischen Einsatzmöglichkeiten von Big Data und Data Analytics. Mangels bislang klar abgesteckter Regelungen besteht die Herausforderung in der Praxis derzeit schon allein darin, den rechtlich zulässigen Spielraum für die Nutzung von verfügbaren Daten und deren Analyse auszuloten.

\subsubsection{Smart Services und Geschäftsmodellentwicklungen in zentralen Lebenswelten}

Smart Services verknüpfen Produkte, Dienstleistungen und Prozesse, die dem Kunden als individualisiertes Gesamtangebot und , as a service ' zur Verfügung stehen (Acatech 2018). Damit sind Smart Services intelligente, digitalisierte Angebote, die durch die Erhebung, Bündelung und Auswertung gesammelter Daten ermöglicht werden. Gebündelt und ausgewertet werden die Daten meist auf digitalen Plattformen, die ortsunabhängig einen übergreifenden Zugang zu den Informationen gewähren und auf denen Anbieter und Nachfrager zusammenkommen (Arbeitskreis Smart Services Welt 2015, S. 21 f.). Durch den datenzentrierten Plattform-Ansatz wird zudem das Andocken weiterer Dienste

\footnotetext{
${ }^{4}$ Die erhobenen Daten müssen auf das für die Zwecke der Verarbeitung notwendige Maß beschränkt sein.

${ }^{5}$ Die Identifizierung der jeweiligen Person darf nur so lange möglich sein, wie es für den Zweck der Datenerhebung notwendig ist.

${ }^{6}$ Personenbezogene Daten müssen für festgelegte, eindeutige und legitime Zwecke erhoben werden und dürfen nicht in einer mit diesen Zwecken nicht zu vereinbarenden Weise weiterverarbeitet werden.
} 
ermöglicht, die aufgrund der intelligenten Datenanalyse individualisiert und damit auf die Situation und den Bedarf des Kunden angepasst werden können.

Im Mittelpunkt der Smart Services steht der Bürger mit seinen Wünschen und Bedürfnissen, die oftmals nicht durch einzelne Anbieter und deren Produkte erfüllt werden können. Zentral für nutzenstiftende Angebote in den Lebenswelten der Bürger ist daher der ganzheitliche Ansatz digitaler Ökosysteme, das sind branchenübergreifende Netzwerke von digital miteinander verbundenen Produktherstellern und Dienstleistern, die gemeinsam Smart Services generieren (Bundesministerium für Wirtschaft und Energie 2017a, S. 4). Der Plattformansatz liefert hierfür die notwendige Infrastruktur und ermöglicht die Kooperationen und vernetzten Systeme. Voraussetzungen für jede Teilnahme als Zulieferer in einem solchen Netzwerk sind standardisierte, schlanke Prozesse, die Bildung von fachlichen und technischen Schnittstellen zu den Ökosystempartnern und letztlich die Akzeptanz der eigenen Austauschbarkeit, weil sich die Netzwerke der Zukunft vermutlich nicht von den Leistungen und Effizienzpositionen bestimmter Zulieferer abhängig machen wollen. Beispiele für Smart Services, die die Bürger in ihren jeweiligen Lebenswelten unterstützen, sind die innovativen datengetriebenen Angebote rund um Smart Home (Lebenswelt Wohnen), Smart Health (Lebenswelt Gesundheit) und Connected Mobility (Lebenswelt Mobilität).

Im Rahmen der Verwertung solcher Smart Services auf Märkten entstehen zunehmend neue Geschäftsmodelle. Dabei ist ein Geschäftsmodell als ein Konzept unternehmerischen Handelns zu verstehen, nach dem mit einem Angebot Mehrwerte für den Kunden und ein Gewinn für den Anbieter realisiert werden können (Grösser 2018). Gegenüber analogen Geschäftsmodellen zeichnen sich digitale Geschäftsmodelle durch eine schnelle Bereitstellung von Lösungen und eine deutlich gesteigerte Umsetzungsgeschwindigkeit aus (Bundesministerium für Wirtschaft und Energie 2017b, S. 18). Das heißt, dass auch Änderungen dem Kunden zeitnah bereitstehen. Als Rückkopplung bieten der einfache Kundenzugang und verbesserte Kundeninformationen wertvolles Potenzial für neue Smart Services. Geschäftsmodelle werden durch die Digitalisierung immer stärker skalierbar und sind dynamisch anpassungsfähig (Seiberth und Gründinger 2018, S. 8). Parallel steigt auch die Geschwindigkeit der Generierung neuer nutzenstiftender Produkte und immer kundenfreundlicherer Prozesse, was wiederum den Wettbewerb erhöht und die Kundenansprüche anwachsen lässt. Die Erwartungen der Kunden an individuelle Lösungen für ihre Wünsche (für die wiederum eine geeignete Datenbasis erforderlich ist) und an eine attraktive ,Customer Journey" passen sich also den Entwicklungen an und erhöhen ihrerseits zunehmend den Entwicklungsdruck. Am Rande sei hier auf das Paradoxon hingewiesen, dass die Kunden immer stärker individualisierte Angebote bekommen 
und erwarten, wofür einerseits die Preisgabe und Nutzung von Daten erforderlich ist, andererseits aber der Datenschutz ein hohes gesellschaftliches Gewicht hat und von den Kunden ebenfalls erwartet wird.

Zurück zu den Geschäftsmodellen: Eine gute Methode, um Geschäftsmodelle zu analysieren, bietet das Businessmodell Canvas. Das Business Model Canvas ist ein vom Schweizer Alexander Osterwalder entwickeltes Konzept, das zur Visualisierung und Strukturierung von Geschäftsmodellen dient. Durch den einfachen grafischen Aufbau dient es als Werkzeug, die wesentlichen Charakteristika von Geschäftsmodellen darzustellen. Es kann insbesondere dazu genutzt werden, innovative Geschäftsmodelle zu beschreiben (Osterwalder und Pigneuer 2010). Der Vorteil des Business Model Canvas liegt darin, dass es universell einsetzbar und damit nicht auf einzelne Branchen oder Geschäftsbereiche beschränkt ist.

Zur Erfassung und Darstellung eines Geschäftsmodells zieht das Business Model Canvas neun Kernelemente heran (siehe Tab. 2.1), deren Ausprägungen und Wechselwirkungen das Geschäftsmodell charakterisieren.

Im Mittelpunkt des Business Model Canvas steht das zentrale Wertangebot an die Kunden. Es beantwortet die Frage, auf welchen Produkten und Services das Geschäftsmodell basiert, um zum einen die Wünsche und Bedürfnisse der Kunden zu befriedigen und sich auf der anderen Seite vom Wettbewerb abzuheben. Aufbauend auf dem Wertangebot werden dazu passende Kundensegmente angesprochen, die sich insbesondere in Endkunden (B2C) und Firmenkunden (B2B) unterscheiden. Darunter können jeweils auch spezielle, weiter differenzierte Kundengruppen adressiert werden. In Abhängigkeit von den Zielkunden sind die Kanäle zu wählen, die für die Kommunikation mit den Kunden und zum Absatz der Leistungen dienen. Auf deren Basis wird die Kundenbeziehung ausgestaltet, die persönlich oder automatisiert gestaltet sein kann. Da das Geschäftsmodell oftmals nicht alleine vollständig umgesetzt werden kann, ist die Zusammenarbeit mit Schlüsselpartnern, wie Lieferanten oder anderen Dienstleistern, notwendig. Mit dem Absatz der Leistungen entstehen Einnahmequellen, die wiederum je nach Produkt und Service verschiedenartig sind. Die Kostenstruktur ist außer durch die Partnerbeziehungen und deren Einnahmeerwartungen insbesondere durch die eigenen Schlüsselaktivitäten und die dafür benötigten Schlüsselressourcen geprägt, zu denen bspw. Personal, Sachmittel und eine Datenbasis gehören. Gerade die Datenbasis ist bei den innovativen digitalen Geschäftsmodellen meistens die zentrale Ressource für die Wertangebote gegenüber den Kunden.

Die nachfolgend diskutierten Geschäftsmodelle rund um die Lebenswelten Wohnen, Gesundheit und Mobilität werden nach einer jeweils grundlegenden 


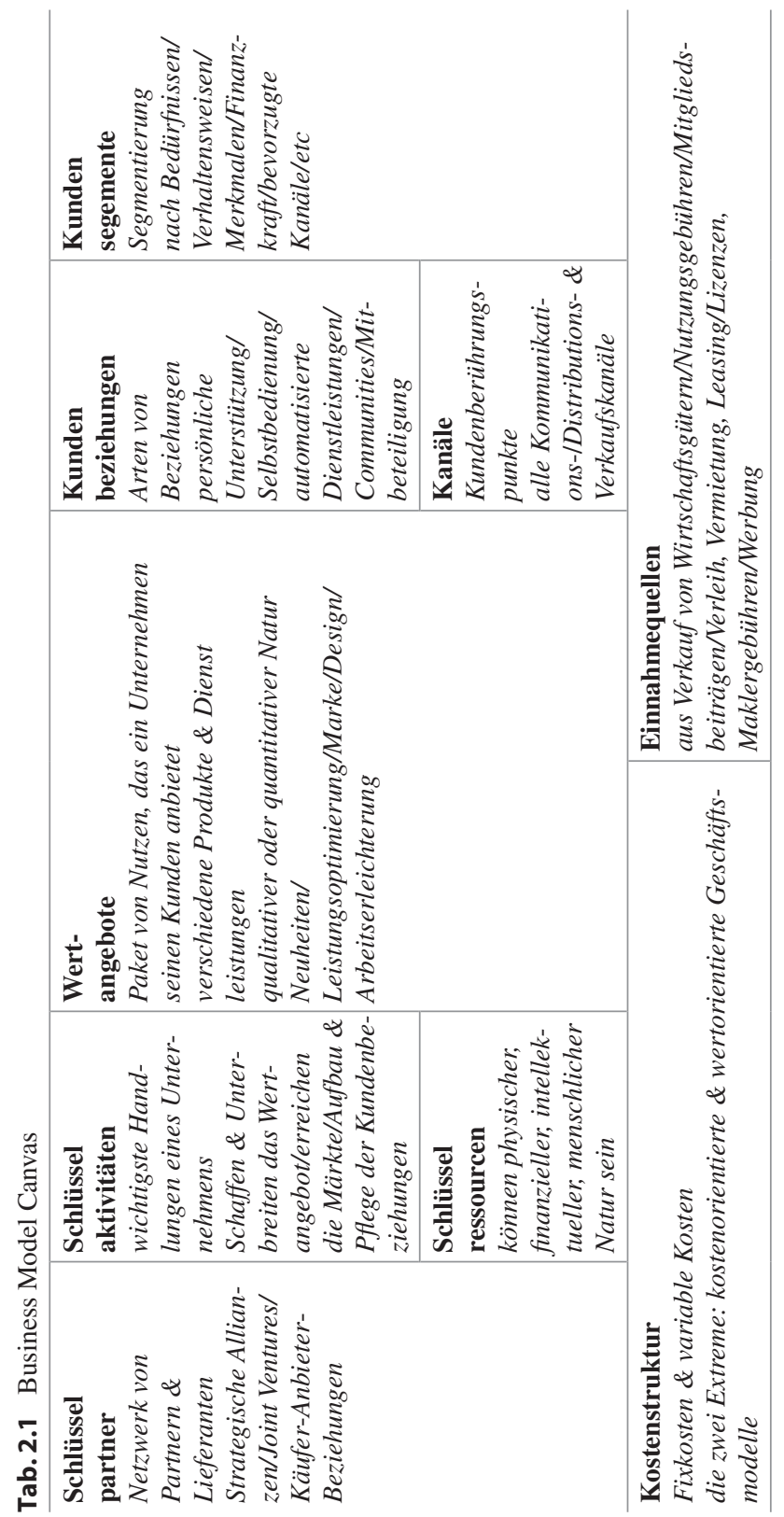


Beschreibung anhand des Business Models Canvas näher analysiert. Auch dabei wird sich zeigen, dass alle diese Geschäftsmodelle als Schlüsselressource auf eine digitale Datenbasis setzen, die für die zentralen Wertangebote benötigt wird.

\subsection{Lebenswelt Wohnen}

\subsubsection{Smart Services im Überblick}

Die Lebenswelt Wohnen umfasst alle Verhältnisse und Aktivitäten innerhalb des häuslichen Umfelds von Bürgern. Darin inbegriffen sind damit die Beschaffenheit (Dach, Fenster etc.), die Ausstattung (Möbel, Haushaltsgeräte etc.) von Haus bzw. Wohnung sowie dessen/deren unmittelbares Umfeld (Garage, Garten etc.). Der Lebenswelt Wohnen können darüber hinaus alle Tätigkeiten zugeordnet werden, die typischer- oder notwendigerweise im Haus oder in der Wohnung durchgeführt werden (bspw. kochen, waschen, bügeln, schlafen, ...). Damit ergibt sich eine Reihe von Anknüpfungspunkten für die Bereitstellung von Smart Services, die im Wohnumfeld zur Steigerung der Lebensqualität eingesetzt werden können (Bundesministerium für Wirtschaft und Energie 2014, S. 13). Ein übergeordneter Begriff für unterschiedliche digitalisierte Angebote in der Lebenswelt Wohnen ist ,Smart Home‘, also die intelligente Vernetzung des eigenen Zuhauses. Dabei gibt es unterschiedliche Entwicklungsstufen, wobei nicht eindeutig definiert ist, ab wann es sich um ein Smart Home handelt. In der letzten Entwicklungsstufe sind alle Geräte, Gegenstände, Materialien etc. miteinander vernetzt, sammeln Daten, werten sie aus und nutzen diese selbstständig mittels Künstlicher Intelligenz zur Optimierung der Wohnumgebung. Durch die intelligente Vernetzung und Auswertung der Daten können zudem Mehrwerte für die Bewohner entwickelt werden. Im Mittelpunkt der Bedürfnisse der Bewohner stehen dabei.

1. die Erhöhung von Lebensqualität durch mehr Komfort,

2. eine erhöhte Sicherheit innerhalb des Hauses sowie der Schutz nach außen und

3. eine möglichst hohe Effizienz bei der Nutzung eingesetzter Ressourcen (Bendel 2018a).

In diese drei Bedürfniskategorien lassen sich auch die Smart Services kategorisieren, wobei eine trennscharfe Abgrenzung nicht immer möglich ist und der größte Mehrwert in der Verknüpfung der verschiedenen Mehrwerte und damit in der Befriedigung aller drei Bedürfniskategorien liegt. 
Smart Services im Bereich Komfort sollen den Bewohner von Alltagsaufgaben entlasten, das Leben in der Wohnung oder im Haus einfacher machen und eine komfortable und gemütliche Wohnatmosphäre schaffen. Anknüpfungspunkte sind z. B. die Regulierung von Luft, Licht und Temperatur, des Zutritts zur Wohnung, der Fensteröffnungen sowie die bequeme, ggf. auch automatisierte Steuerung verschiedener Komponenten und Gegenstände. So ist bei vielen Beleuchtungssystemen mittlerweile eine per App steuerbare Veränderung der Helligkeit und der Lichtfarbe möglich, inkl. des An- und Abschaltens des Lichts aus der Ferne. Ein weiteres Beispiel ist die intelligente Steuerung der Heizung, wie sie mittlerweile von einer ganzen Reihe von Herstellern angeboten wird. Dabei kann die Raumtemperatur wiederum entweder manuell per App oder Sprachsteuerung geregelt werden, oder aber die Einstellung erfolgt automatisiert, indem Daten per Funk, über Wandsensoren, durch Geofencing oder mittels Einsatzes einer Künstlichen Intelligenz weitergeleitet und für die Steuerung eingesetzt werden. Das Geofencing sammelt Standort- und Bewegungsdaten über GPS und erkennt, wenn die Bewohner zu Hause sind und wo sie sich aufhalten; demnach wird die Temperatur in den entsprechenden Heizzonen angepasst. Verlassen die Bewohner das Haus, wird die Temperatur automatisch abgesenkt. Befinden sie sich auf dem Heimweg, springen die Thermostate an und adjustieren die Heizung (Innogy 2017). Dabei kann zusätzlich über eine App oder andere Steuerungsmechanismen nachgeregelt werden. Wird zudem eine Künstliche Intelligenz verwendet, können Daten über die Gewohnheiten der Bewohner gesammelt und die Temperaturregelung selbstlernend angepasst werden. Ergänzend können auch weitere Sensoren zum Einsatz kommen, die zu hohe Luftfeuchtigkeit registrieren und z. B. mit einem Schimmelwarnungssystem ausgestattet sind.

Wie sich zeigt, erhöht diese den Komfort adressierende Lösung zudem die Ressourceneffizienz, indem die Energienutzung optimiert und z. B. vermieden wird, dass die Heizung im Fall einer längeren Abwesenheit der Bewohner unnötig viel Öl, Gas oder Strom verbraucht. Durch die Vernetzung und den Datenaustausch mit Fenster- und Türsensoren kann zudem ein Energieverlust beim Lüften verhindert werden. Darüber hinaus können zahlreiche weitere Daten gesammelt werden, die wertvolle Informationen über den Energieverbrauch bieten und Verbesserungspotenziale aufzeigen. Insbesondere sog. ,Smart Meter" (intelligente Zähler) können den Bewohnern in einem Smart Home entsprechende Mehrwerte schaffen. So wird etwa ein intelligenter Stromzähler mit den verschiedenen Geräten, die an das Stromnetz angeschlossen sind, vernetzt und über ein Gateway zur Datenübertragung dazu befähigt, alle Verbrauchsdaten nicht nur gesamthaft, sondern auch differenziert nach den Einzelgeräten auszulesen und zu analysieren. 
Der Nutzer erhält sodann über seine Smart-Home-App ausgewertete und visuell aufbereitete Informationen über seinen Stromverbrauch. Ähnlich differenziert kann über Smart Meter auch der Öl-, Gas- und Wasserverbrauch analysiert werden. Im Ergebnis lassen sich verbrauchsintensive Einrichtungen wie bspw. wiederum die Heizung oder eine Bewässerungsanlage im Garten optimiert steuern. Auch die automatische Deaktivierung von Elektronikgeräten in den Stand-byModus hilft, Betriebskosten zu senken und kommt damit überdies dem ökologischen Umgang mit Energieressourcen entgegen.

Ein weiterer Smart Service entsteht durch die Nutzung von FotovoltaikAnlagen: Der Nutzer wird über den aktuellen Stand der Stromerzeugung seiner Anlage informiert. Wird bei entsprechendem Wetter gerade ausreichend Strom produziert, kann er z. B. ad hoc kostengünstig seine Wäsche waschen (Strom Magazin 2018). Dieses Konzept verfolgt beispielsweise der Smart Meter-Hersteller Discovergy über die Kooperation mit der Smart Home-App iHaus: Ein Ampelsystem zeigt dem Kunden auf dem Smartphone an, wann Strom besonders günstig bereitsteht. Außerdem kann der Nutzer ,Wenn-dann-Regeln“ aufstellen. Damit steuert er seine Geräte über ihre Beziehungen untereinander. Beispielsweise wird zunächst das Elektroauto bis zur gewünschten Kapazität aufgeladen und der Geschirrspüler nur dann gestartet, sollte noch genügend günstige Energie verfügbar sein (Discovergy 2018). Gleichermaßen kann das vernetzte Smart Home an den Energieversorger zum Datenaustausch angebunden werden. Dadurch können Energieflüsse besser prognostiziert und die Versorgungssicherheit gesteigert werden.

Im Bereich der Sicherheit decken Smart Services vorrangig den Einbruchschutz und die haus- bzw. wohnungsinterne Schadensprävention ab. Neben kamerabasierten Zugangskontrollen und Überwachungssystemen innerhalb und außerhalb des Wohnraums sind es WLAN-Schlösser, die sich per App öffnen und schließen lassen, sowie Alarmsysteme, die mit direkten Dienstleistungen gekoppelt sind. Auch die oben beschriebenen Lichtsysteme lassen sich zum Schutz vor Einbrechern einsetzen. Dies kann insbesondere durch sog. Anwesenheitssimulatoren geschehen, die das Verhalten der Bewohner erlernen und bei deren Abwesenheit das Licht analog der üblichen Gewohnheiten steuern.

Ein Anwendungssystem für Smart Services im Bereich der Schadenprävention sind sensorbasierte Angebote zur frühzeitigen Aufdeckung von Wasserschäden. So können Sensoren in den Wohnräumen installiert werden, die ungewöhnliche Nässe melden und durch ein Abschalten der Wasserversorgung vor Durchfeuchtung oder Überschwemmung schützen. Zudem bietet bspw. Grohe ein System an, das bereits in den Wasserrohren Mikroleckagen erkennt und so frühzeitige Präventionsmaßnahmen ermöglicht (Grohe 2018). 
Neben klassischen Smart Home-Anwendungen, die im privaten Wohnraum allen möglichen Nutzern zugutekommen, können auch intelligente Assistenzsysteme (Ambient Assisted Living) zu den Smart Services in der Lebenswelt Wohnen gezählt werden, die insbesondere für Senioren und pflegebedürftige Menschen entwickelt wurden. Dabei geht es um Anwendungen, die diese Zielgruppen im Haushalt unterstützen und ein selbstbestimmtes Leben im gewohnten und vertrauten Umfeld gewährleisten sollen (SmartHome Initiative Deutschland und $\mathrm{mm} 1$ o. J., S. 5 f.). Die Angebote reichen dabei von Notrufmelde-systemen über elektronische Gesundheitsdienste bis hin zu Pflegerobotern. Der Übergang zur Lebenswelt Gesundheit ist an dieser Stelle fließend (Siepermann 2018).

Eine wichtige technologische Entwicklung, die in vielen Lebenswelten Anwendung findet, ist die Sprachsteuerung: Im Bereich Wohnen sind es Sprachassistenten wie Google Home und Amazon Alexa, die die Bedienbarkeit des vernetzten Zuhauses erleichtern und für den Nutzer komfortabler gestalten. Allerdings ist es in den meisten Fällen weiterhin erforderlich, dass Anweisungen und Vorgaben durch den Menschen vorgenommen werden. Die zukünftigen Entwicklungen im Smart Home-Bereich forcieren jedoch zunehmend die eigenständige Kommunikation der Komponenten untereinander, sodass sich das Smart Home eigenständig und automatisiert steuert und menschliches Zutun nicht mehr erforderlich ist. Dazu ist es notwendig, Daten aller Geräte und Komponenten kontinuierlich zu sammeln und über eine gemeinsame Plattform geeigneten Anwendungsdiensten zur Verfügung zu stellen. Nur so kann eine intelligente Regulierung z. B. der Heizungswärme oder des Einsatzes der Waschmaschine mit Strom aus der Fotovoltaikanlage automatisiert auf Basis vorhandener Wetterdaten erfolgen. Ziel ist es, dass die smarten Systeme aus vergangenen Ereignissen lernen und entsprechende Handlungsweisen daraus selbstständig ableiten - und nach und nach optimieren. Bereits heute können regelmäßig wiederkehrende Aktionen einzelner Einrichtungen im Haushalt automatisiert gesteuert werden (z. B. zentrale Heizungsanlagen mit Zeitschaltuhren oder Temperaturfühlern). Jedoch ist es aktuell noch nicht möglich, verkettete Systeme ereignisbasiert handeln zu lassen, da nach aktuellem Stand die Datenquellen und Geräte bzw. Einrichtungen noch nicht ausreichend miteinander verknüpft sind. Zudem existieren bislang noch kaum hinreichend intelligente Dienste, um die Daten entsprechend auszuwerten und daraus Handlungsweisen ableiten zu können.

Im Folgenden werden zwei bereits real existierende Geschäftsmodelle beschrieben, die sich in Smart Services rund um die Lebenswelt Wohnen eingliedern. Dabei steht der Nutzen der Daten im Vordergrund, auf deren Basis Wertangebote für die Bewohner in einem ,Smart Home` erzeugt werden. 


\subsubsection{Ausgewählte Geschäftsmodelle innerhalb der Smart Services}

\subsubsection{Amazon Key}

\section{Beschreibung des Geschäftsmodells}

Mit dem Service „Amazon Key“ bietet Amazon ein schlüsselloses Zugangssystem zu Wohnungen/Häusern an, durch das der Nutzer dritten Personen aus der Ferne die eigenen Räumlichkeiten öffnen kann. Damit kann zum einen der Zutritt für Familienangehörige und Freunde, zum anderen das Erbringen von Dienstleistungen in den Räumlichkeiten der Nutzer ohne deren Anwesenheit ermöglicht werden (Heise online 2017). Für diesen Zweck wird die Haustür mit einem smarten Türriegel versehen, der sich mittels der zugehörigen Amazon KeyApp programmieren lässt. Zusätzlich enthält das Angebot eine ebenfalls per App steuerbare Überwachungskamera, mithilfe derer der Eingangsbereich bei jeder Türöffnung aufgezeichnet wird. So kann der Nutzer z. B. der Haushaltshilfe, dem Dogsitter oder einem Handwerker temporären Zugang zu seinem Wohnraum verschaffen. Über die Amazon-Key-App stellt der Nutzer der entsprechenden Person einen Code zur Verfügung, mit dem die Tür innerhalb eines bestimmten Zeitraums geöffnet werden kann. Wenn der Zugang erfolgt, bekommt der Hausbewohner eine Benachrichtigung auf sein Handy. Eine Kontrolle ist dann durch Einsicht in die Aufzeichnung des Eingangsbereichs ohne Weiteres möglich.

Amazon selbst schafft sich mit Amazon Key die Voraussetzungen und die Möglichkeit einer, in-home'-Paketlieferung, die Amazon-Prime-Kunden exklusiv angeboten wird. Der Paketbote erhält dafür bei Auslieferung eine Authentifizierung, kann hiermit das smarte Türschloss öffnen und das Paket in der Wohnung des Nutzers abstellen. Der Nutzer kann mit diesem Service nicht mehr nur den Lieferstand ,tracken', sondern sich zudem die Lieferung selbst über das aufgezeichnete Video ansehen (Amazon 2018).

Die erhobenen Daten rund um Amazon Key umfassen Verhaltens- inkl. Konsuminformationen über die Bewohner, indem übermittelt wird, wann der Nutzer zu Hause ist, wann er Besuch empfängt und welche Produkte und Dienstleistungen er bei welchen Anbietern in Anspruch nimmt. Basierend darauf lassen sich weitere Mehrwertdienste anbieten, die sich in das Angebot von Amazon Key integrieren lassen. Daneben werden Videodaten der Überwachungskamera gesammelt, die bei Amazon gespeichert werden, um dem Nutzer bei Bedarf zur Verfügung zu stehen.

Überdies kann Amazon Key ein massives Kostenproblem für Paketzustelldienste lösen, denen Mehrfachanfahrten oder Zusatzwege zu alternativen 
Lieferungsorten erspart werden. Alternativ kommt es in der Realität immer wieder vor, dass Pakete bei Abwesenheit der Bewohner von den Zustellern einfach vor die Tür gestellt werden und dann abhandenkommen. Auch das wird durch Amazon Key verhindert - und erhöht die Sicherheit des Paketerhalts bzw. vermeidet Verlustschäden. Indem die Türöffnungsfunktion auch für andere Zusteller jenseits von Amazon-Lieferanten nutzbar werden soll, erhält Amazon auch Daten über die Bestellung bei Drittanbietern und über deren Zustellmodalitäten, damit also ggf. auch von Konkurrenten.

Bis auf Weiteres ist Amazon Key eine singuläre Smart Home-Anwendung, ohne Vernetzung mit anderen Funktionen, die zu gesamtheitlichen Lösungen führen könnten. Amazon Key bietet aber wichtige Ansatzpunkte und Informationen auf dem Weg dorthin und kann mit der Möglichkeit zur Verknüpfung der gewonnenen Daten mit weiteren Daten aus der Wohneinheit einen Meilenstein zu einem ganzheitlich vernetzten Smart Home darstellen.

\section{Geschäftsmodellanalyse nach dem Business Model Canvas}

Das Wertangebot von Amazon Key ist die App-basierte Zugangskontrolle für dritte Personen zum eigenen Wohnraum. Dadurch können Dienstleistungen flexibler in Anspruch genommen und Zeiten effizienter genutzt werden. Ziel des Angebots ist es, Familienmitgliedern, Freunden und Nachbarn sowie Dienstleistern jederzeit und aus der Ferne kontrollierten Zugang zu ermöglichen und den Wohnraum während des temporären Zugangs Dritter zu überwachen. Durch die jederzeitige Lieferung der bestellten Ware in den eigenen Wohnbereich kommt es nicht mehr zur Paketzustellung an andere Orte, wie dem Nachbarn oder einem nahegelegenen Shop, sodass dies zu Convenience und Zeitersparnis bei den Kunden führt. Im Kundensegment ist Amazon Key auf den B2C-Bereich fokussiert, da das Angebot primär auf den Privathaushalt zugeschnitten ist. Was den Lieferdienst anbetrifft, sind die Zielgruppe in erster Linie Personen mit Affinität zum Onlinehandel (Amazon 2018). Als Distributionskanal bedient sich Amazon Key verschiedener Medien wie einer App, der Homepage, dem Telefon und der E-Mail. Die Kundenbeziehung ist durch eine hohe Standardisierung und zudem technisch geprägt, da die Anwendung mithilfe der App gesteuert wird. Es gibt zudem kaum einen direkten Kundenkontakt. Bei einer Bestellung im Amazon Store sind die üblichen Self-Service-Optionen integriert, die um die Option der Inhouse-Lieferung erweitert sind. Einnahmequellen ergeben sich zunächst aus den einmaligen Gebühren für die Anschaffung von Amazon Key in Höhe von derzeit 212 EUR. Darin enthalten sind neben der für die Nutzung erforderlichen App die Kamera und das smarte Türschloss (Ignor 2018). Da Amazon Key zunächst nur für Prime-Mitglieder verfügbar ist, sind zusätzlich Mitgliedsgebühren mit 
dem Angebot verknüpft. Ebenfalls können sich die Kundenzahl im herkömmlichen Online-Handelsgeschäft und die Bestellintensität bei Amazon durch die angebotenen Mehrwerte erhöhen, sodass weitere Einnahmen erschlossen werden.

Die Schlüsselaktivitäten zur Umsetzung des Geschäftsmodells liegen in der Bereitstellung der technischen Geräte sowie der App und in den damit geschaffenen Zugangs- und Kontrollfunktionen. Es ist zwingend notwendig, dass das smarte Türschloss und die Kamera einwandfrei arbeiten. Ebenfalls ist die Absicherung gegen mögliche Einbrüche aufgrund digitaler Sicherheitslücken essenziell. Dafür sind ggf. laufende Updates erforderlich, wenn Schwachstellen erkannt werden. Des Weiteren können die erfassten Verhaltens- und Konsumdaten der Bewohner genutzt werden, um über die eigentliche Funktionalität von Amazon Key hinaus weitere Mehrwertleistungen anbieten zu können. $\mathrm{Zu}$ den Schlüsselpartnern zählen die Hersteller der smarten Türschlösser, bei denen der Nutzer zwischen Schlage, Yale oder Kwikset wählen kann. Ebenfalls sind Paketdienstleister, über die eigene Produkte ausgeliefert werden, Partner. Ergänzend arbeitet Amazon mit ca. 1300 Servicepartnern zusammen, das sind u. a. Hundesitter, Handwerker oder Reinigungsunternehmen (Kapalschinski 2017). Schlüsselressourcen sind neben dem smarten Türschloss und der Überwachungskamera vor allem die gesammelten Daten über die Nutzer und deren Anwesenheitsverhalten, Besucher- und Dienstleisterbeziehungen sowie Konsummerkmale. Diese Daten und die damit generierten Informationen ermöglichen es Amazon, das Angebot für die Kunden stetig zu verbessern und immer individueller auszugestalten (Siethoff 2017). Soweit Amazon auch in die interne Lebenswelt Wohnen, bspw. über Alexa, Einblick erhält, ist die Kombination dieser Informationen mit denen aus Amazon Key zentral, um künftig noch umfassendere Offerten kreieren zu können. Damit schließt sich wiederum der Kreis zu möglichen neuen Wertangeboten.

\subsubsection{Ubtech Robotics Lynx}

\section{Beschreibung des Geschäftsmodells}

,Lynx “ ist der Roboter des chinesischen Robotic-Herstellers Ubtech, der seit 2017 auf dem Markt angeboten wird. Der datengesteuerte Roboter ist ca. $40 \mathrm{~cm}$ groß, kann über eingebaute Mikrofone und Lautsprecher hören und sprechen und sich selbstständig fortbewegen. Ubtech arbeitet mit Amazon zusammen und verwendet die von Amazon entwickelte Sprachsteuerungsfunktion Alexa. Dadurch kann Lynx automatisch Sprache erkennen und anhand seiner Verständnisfunktion antworten (Wollaston 2017). Zudem ist der Kopf des Roboters mit einer Kamera ausgestattet, die sich mithilfe von Gelenkmotoren drehen, senken und erhöhen lässt und dadurch Bildmaterial aus der Umgebung vollständig erfassen und 
aufnehmen kann (Ubtech 2018). In Kombination mit der integrierten Gesichtserkennungsfunktion kann Lynx ausmachen, wer sich in der Wohnung befindet, bekannte Personen mit Namen ansprechen und mit diesen in Interaktion treten. Im Aktivitätsmodus kann der Roboter selbstständig die Kommunikation aufnehmen, er kann aber auch per App dazu aufgefordert werden, mit den Personen seines Umfelds Kontakt aufzunehmen. Dies erfolgt mittels einer Avatar-Funktion, die den Roboter als Übertragungsschnittstelle nutzt und eine Zweiwegekommunikation ${ }^{7}$ ermöglicht. So kann der Anwender des Roboters auch Kontakt mit Personen aufnehmen, die sich im gleichen Raum mit Lynx befinden, ohne dass er selbst anwesend sein muss. Neben der Sprachkommunikation kann auch Bildmaterial ausgetauscht werden. Des Weiteren sind im Brustbereich Infrarotsensoren eingebaut, mittels derer Bewegungsdaten gesammelt und ausgewertet werden können (Ubtech 2018).

Durch die Schnittstelle zur Alexa-Sprachsteuerung lassen sich unterschiedliche Aktivitäten innerhalb des Smart Home steuern. So kann Lynx - ebenso wie Alexa auch - nach Aufforderung die Rollos nach unten fahren, Musik abspielen und Tätigkeiten wie Terminvereinbarungen, Schreiben von E-Mails und Berechnungen von optimalen Verkehrsrouten übernehmen. Ebenfalls ist der Roboter mit einer Künstlichen Intelligenz ausgestattet, durch die er ständig dazulernen und seine Funktionen verbessern kann (Lacace 2017). Außer rein sprachlich kann Lynx auch körperlich mit seinem Umfeld in Interaktion treten. Bspw. kann er im Bereich Ambient Assisted Living (AAL) ältere oder in ihrer Bewegungsfreiheit eingeschränkte Menschen im Alltag unterstützen oder zur Unterhaltung oder für verschiedene Aktivitäten eingesetzt werden. Damit wird Lynx zu einem umfassenden (Smart Home-)Assistenten.

Jenseits der physischen Funktionen kann er auch für geistiges Training, bspw. für an Demenz erkrankte Personen, genutzt werden. Ein wichtiges Anwendungsfeld ist zudem die Unterstützung beim Einbruchschutz und die Erhöhung der Sicherheit zu Hause. So kann sich Lynx eigenständig in festgelegten Bereichen des Hauses bewegen und über die integrierten Infrarotsensoren Bewegungen innerhalb des Hauses erkennen, diese seinem Nutzer melden und sofort Bildmaterial der auffälligen Umgebung übermitteln. Auf diese Weise kann der Roboter als mobile Überwachung der Wohnung eingesetzt werden. Neben der

${ }^{7}$ Zweiwegekommunikation meint die Kommunikation, bei der eine Botschaft nicht nur vom Sender zum Empfänger, sondern ebenso vom Empfänger zum Sender übertragen werden kann (zweiseitige, bilaterale Kommunikation). 
Sicherheit dient Lynx vor allem dem Komfort: Er unterstützt seinen Anwender bei einfachen und alltäglichen Aufgaben zu Hause, unterhält ihn und dient als Steuerungszentrale des Smart Home (Business Panorama 2017).

\section{Geschäftsmodellanalyse nach dem Business Model Canvas}

Das Wertangebot von Lynx besteht in der Unterstützung bei verschiedenen Tätigkeiten in der erweiterten Lebenswelt Wohnen: Abläufe im Alltag werden vereinfacht und beschleunigt, es sind Unterhaltungsfunktionen enthalten und im Überwachungsmodus ist eine bewegungs- und videodatenbasierte Überwachung möglich, im Rahmen derer sich der Roboter auch aus der Ferne steuern lässt. Es handelt sich bei Lynx damit um einen smarten Assistenten, der nicht nur das Smart Home steuert, sondern das Leben zu Hause erleichtert. Durch die Kombination von Robotertechnik und digitaler Sprachassistenten-Funktionen wird eine persönliche Schnittstelle zwischen Mensch und Maschine geschaffen. Die Serviceleistungen von Lynx richten sich an das B2C-, aber durchaus auch an das B2B-Kundensegment, da Lynx sowohl im privaten Zuhause als auch in sonstigen Umgebungen einsetzbar ist. Im B2B-Bereich kann Lynx bspw. in Altenheimen oder Kindergärten genutzt oder zur Überwachung von Bürogebäuden oder Industrieanlagen eingesetzt werden. Der Distributionskanal ist vor allem der Online-Verkauf. Bei Amazon ist Lynx für 800 US\$ bestellbar. Aktuell wird der Roboter nur in den USA verkauft; weitere Länder sollen aber zeitnah folgen. Die zugehörige App ist im Google Playstore und im App Store von Apple kostenfrei erhältlich (Ubtech 2018). Die Kundenbeziehung ist vor allem durch die persönliche Interaktion mit Lynx gekennzeichnet. Außerdem kann der Kunde über die App mit dem Hersteller in Verbindung treten. Daneben ist der Kundenservice per E-Mail und Telefon erreichbar. Nicht nur Ubtech, sondern auch Amazon steht mit dem Kunden in enger Beziehung. Alle Daten, die im Zusammenhang mit der Alexa-Nutzung entstehen, laufen über das Rechenzentrum von Amazon und dienen dem Unternehmen als Kundenschnittstelle. Primäre Einnahmequelle ist zunächst der (jeweils einmalige) Verkaufspreis des Roboters. Jedoch steht derzeit die Monetarisierung von Lynx nicht im alleinigen Fokus. Insbesondere geht es darum, den Markt zu erobern, Wettbewerbsvorteile zu erzielen und die Robotertechnik sowie die enthaltene Künstliche Intelligenz zu verbessern. Mit steigenden Nutzerzahlen werden nicht nur höhere Einnahmen generiert, sondern es können vor allem auch mehr Daten gesammelt und ausgewertet werden, die zu einer Verbesserung der Leistungen nutzbar sind.

Durch die Datensammlung sind sowohl Amazon als auch Ubtech in der Lage, die Kommunikation, das Wohn- und Kaufverhalten, die Mediennutzung etc. jedes Nutzers besser zu verstehen. Mittels entsprechender Datenanalyse können 
somit über passende Kaufempfehlungen mehr Umsatz generiert und die Möglichkeit verbessert werden, neue Mehrwerte zu erzeugen. Schlüsselaktivitäten sind die (Weiter-)Entwicklung von Robotics und der Künstlichen Intelligenz. Dies geschieht durch die Sammlung und Auswertung aller Nutzerdaten, die über die Interaktion mit Lynx anfallen und im Rahmen der Kundenprofilanalyse analysiert werden. Lynx liefert damit umfassende Daten über das Verhalten und die Bedürfnisse seiner Nutzer und generiert damit ein umfangreiches Kundenverständnis, auf Basis dessen immer weitere Services angeboten werden können. Neben dem Schlüsselpartner Amazon arbeitet Ubtech mit verschiedenen Universitäten und Forschungseinrichtungen zusammen (Industriepraxis 2018). Die gewonnenen Kunden-, Umgebungs- und Kommunikationsdaten stellen die Schlüsselressourcen dar. Gerade in Bezug auf den Überwachungsmodus sind zudem die Bewegungsdaten und Videoaufzeichnungen essenziell. Die möglichst umfassende Kenntnis der Lebensumstände und der Präferenzen seiner Anwender ist die Grundlage für das Wertangebot von Lynx und dessen weitere Entfaltung.

\subsubsection{Spannungsfeld Datennutzung und Datenschutz}

Services in den Bereichen Smart Home und Ambient Assisted Living unterstuitzen das Leben im häuslichen Umfeld mit dem Ziel, ein höheres Maß an Sicherheit, Komfort und Effizienz für die Bewohner herzustellen. Dies geschieht beispielsweise durch Automatisierung von Warnungen, Arbeitsabläufen und physischer Hilfe. Dabei entfaltet das vernetzte Zuhause sein volles Potenzial erst dann, wenn möglichst viele Daten über die Einrichtungen, Geräte und deren Nutzer ausgelesen und analysiert werden. Erst dadurch entsteht ein umfassendes Bild über den Menschen in seinem Zuhause und können individuell passende (Unterstützungs-)Leistungen angeboten bzw. in Anspruch genommen werden.

Die Lebens- und Wohnqualität kann insbesondere für ältere Menschen gesteigert werden. Mit dem Eintritt in den Massenmarkt können Smart Home-Komponenten das sichere und altersgerechte Wohnen fördern (Nationaler IT-Gipfel 2015). Das häufig wichtigste Ziel älterer Menschen, so lange wie irgend möglich selbstständig und selbstbestimmt zu leben (Bundesinstitut für Bevölkerungsforschung 1996), wird dadurch gefördert. Insgesamt hilft das Smart Home, die gesellschaftliche Entwicklung eines möglichst individualisierten Wohnangebots je nach persönlichen Vorlieben in Bezug auf Wohnumgebung und Verfügbarkeiten von Assistenzsystemen umzusetzen (Hartmut et al. 2010, S. 11).

Neben allgemeinen Daten zur Person, wie Alter, Geschlecht, Authentifizierungs- und Kontaktdaten, die in aller Regel bereits bei der Anmeldung zu Smart 
Services preisgegeben werden, sind es im Wohnbereich vor allem Verhaltensund Nutzungsdaten sowie Überwachungsdaten (Bild und Ton), die erhoben und ausgewertet werden. Aber auch Standort- und Bewegungsdaten werden in der Lebenswelt genutzt, um Sicherheit (bewegt sich etwas im Haus, obwohl der Bewohner nicht daheim ist?), Komfort (die Heizung schaltet sich ein, wenn sich der Bewohner auf dem Nachhauseweg befindet) und Effizienz (alle Lampen schalten sich aus, wenn der Bewohner das Haus verlässt) zu generieren. Essenziell für Smart Services, die im Bereich Wohnen das Bedürfnis nach Sicherheit adressieren, sind die Überwachungs- und insbesondere Videodaten. Durch Aufzeichnung der Überwachungsdaten (Bild und Ton) mittels verschiedener Systeme, wie Kameras und Bewegungssensoren, können Auffälligkeiten erkannt, analysiert bzw. verifiziert und weiterverarbeitet werden. In Kombination mit der Möglichkeit, Daten automatisiert auszuwerten und im Notfall weitere Schritte einzuleiten, liegt eine effektive Hilfestellung für die Betroffenen vor, insbesondere indem Maßnahmen in Akutfällen auch ohne deren aktives Eingreifen eingeleitet werden können.

Zudem kann die Sicherheit präventiv durch die Analyse von Verhaltens- und Nutzungsdaten gesteigert werden, indem die Erkenntnisse bspw. zur automatisierten Anwesenheitssimulation dienen. Auch unter Komfort- und Effizienzerwägungen sind gerade die Verhaltens- und Nutzungsdaten interessant. Je umfangreicher Informationen über Gewohnheiten der Bewohner eines Smart Home vorliegen, desto genauer können Leistungsangebote auf deren Bedürfnisse zugeschnitten werden. Erst das Wissen über die Uhrzeit des Aufstehens (auch in Echtzeit, z. B. durch Konnektivität mit einem smarten Wecker) ermöglicht das rechtzeitige Erwärmen des Badezimmers und die automatisierte Ingangsetzung der Kaffeemaschine und des Toasters. Benötigt werden dafür fast ausschließlich sensible personenbezogene Daten (Akoto 2018), die besonderen Datenschutzbestimmungen unterliegen. Jedoch sind gerade diese Daten aufgrund des engen Bezugs zu den Gewohnheiten und Bedürfnissen der Menschen von besonderem Interesse für die Entwicklung smarter Geschäftsmodelle. Damit handelt es sich auch um Daten und Informationen, die eben nicht frei zugänglich sind, sondern einen direkten Zugang zum Nutzer und dessen Einverständnis zur Erhebung voraussetzen, um ein hinreichend treffendes Bild über das Privatleben zu gewinnen. Durch die Analyse dieser Daten können Geschäftsmodelle nutzerzentriert weiterentwickelt und neue Angebote und Services konzipiert werden.

Aufgrund des Umfangs und der Sensibilität der im Wohnbereich erhobenen Daten gibt es verschiedene Gesetze, die aus Daten- und Verbraucherschutzgründen für den Bereich Smart Home Anwendung finden und damit auch Anwendungsrestriktionen setzen. Dazu zählen u. a. das Telekommunikationsgesetz (TKG), das 
Telemediengesetz (TMG), das Energiewirtschaftsgesetz (EnWG) und das Gesetz zur Digitalisierung der Energiewende. Des Weiteren stellt die seit 2018 gültige Europäische Datenschutzgrundverordnung (EU-DSGVO) persönliche Daten unter einen strengen und komplexen Schutz. Die neue EU-DSGVO regelt Vorschriften für die Erhebung und Verwertung personenbezogener Daten, die auch für das Smart Home umfassend Gültigkeit besitzen (Bundesverband Digitale Wirtschaft 2018a, S. 6 f.). Mit der EU-DSGVO drohen empfindliche Bußgelder, wenn Unternehmen gegen gültige Datenschutzbestimmungen verstoßen. Dabei gelten die folgenden Grundsätze:

- Die Erhebung personenbezogener Daten bedarf einer Legitimation durch einen gesetzlichen Erlaubnistatbestand oder durch Einwilligung des Nutzers.

- Die Einwilligung muss freiwillig erfolgen, d. h. die Nutzung eines Geräts darf nicht an die Einwilligung gekoppelt sein. Das fällt bei vernetzten Smart Home-Geräten in der Praxis schwer, da sie ja faktisch nur genutzt werden können, wenn sie Daten erheben.

- Der Einwilligende muss umfassend über die Datenerhebung informiert werden.

- Die Datennutzung darf nur in den Grenzen der Einwilligung erfolgen. Das Sammeln von Daten , auf Vorrat' ohne Zweckbindung ist damit untersagt.

- Der Grundsatz der Datenminimierung steht im Vordergrund der EU-DSGVO. Damit dürfen nur Daten erhoben werden, die für einen definierten Zweck erforderlich sind.

- Alle Geräte und Anwendungen müssen so konzipiert (,Privacy by Design") und voreingestellt (,Privacy by Default ${ }^{\circ}$ ) sein, dass möglichst wenig personenbezogene Daten erhoben werden. Ein Ausweg besteht nur über die Anonymisierung der Daten (Verordnung des Europäischen Parlaments und des Rates 2016/679 2018).

Eine Herausforderung im Zusammenhang mit dem Smart Home besteht darin, dass Nutzer oftmals nicht sicher sind und sein können, welche Daten über die verwendeten Geräte aufgezeichnet werden und wofür diese tatsächlich genutzt werden. Ein Beispiel ist das Sprachsteuerungssystem Alexa, das laut Hersteller-Angaben Audiodateien erst dann aufzeichnet, wenn der Nutzer Alexa anspricht. Um Alexa zu verstehen, muss jedoch eine gewisse Aktivität des Geräts zu jedem Zeitpunkt sichergestellt sein. Ähnlich verhält es sich mit Überwachungssystemen, die Audio- oder auch Videodaten aufzeichnen. Über die Netzwerkanbindung können solche Daten direkt von den angebundenen Unternehmen verwertet werden. Vor diesem Hintergrund haben viele Bürger nach wie 
vor eine hohe Skepsis gegenüber einer umfassenden Datenpreisgabe und Datenauswertung in der sehr privaten Lebenswelt Wohnen. Verstärkt wird die Sorge dadurch, dass insbesondere die datengetriebenen ,BigTechs', die mit ihren individuellen Smart Services weltweit führend sind, außerhalb der EU in den USA (Amazon, Apple, Facebook, Google) und China (Baidu, Alibaba, Tencent) sitzen, wo andere, viel liberalere Datenschutzregeln gelten. Das Datenschutzklima und der rechtlich fixierte Datenschutz dürften von daher eine größere Verbreitung der Smart Home-Technologien in Deutschland und Europa bislang bremsen (Deloitte 2018, S. 25 ff.).

Ansätze zur Lösung und zur Fortentwicklung von Smart Services in der Lebenswelt Wohnen liegen in der Sicherung der Systeme und der Hoheitsrechte über sensible personenbezogene Daten. Gerade vernetzte Haussysteme, die Sicherheits- und deshalb Überwachungssysteme beinhalten, müssen zudem ausreichend Schutz gegen Hackerangriffe bieten und manipulationssicher sein. Es ist unerlässlich, dass die Geräte über verschlüsselte Netzwerke miteinander kommunizieren und immer automatisch die neuesten Updates aufgespielt werden, um entstehende Sicherheitslücken möglichst unverzüglich $\mathrm{zu}$ schließen. Auch die Datenübertragung nach außen, zum Server bzw. in die Cloud (und wieder zurück), muss verschlüsselt und entsprechend gesichert erfolgen. Was die Hoheitsrechte anbetrifft, ist rechtlich sicherzustellen, dass der Bürger selbst als Nutzer von Smart Services entscheidet, welche Daten von ihm und seinem Wohnumfeld erhoben werden und welche Teile davon er wem für welche Zwecke zur Verfügung stellt - aber dafür muss er auch die Möglichkeit entsprechender Wahlhandlungen bekommen, unabhängig vom Hersteller der vernetzten Geräte oder vom Plattformbetreiber.

Herausforderungen entstehen ferner durch das Tempo, mit dem Internetkonzerne und Start-ups datengetriebene Services auf den Markt bringen. Oftmals können Politik und Regulierung mit dieser Geschwindigkeit nicht schritthalten (Heide 2018). Um umfassende Smart Services in den Lebenswelten bieten zu können, müssen das Verständnis aufseiten der Bürger erhöht und Risiken möglichst minimiert werden, um auch die Chancen zu wahren und zu realisieren. Die Chancenwahrung wiederum steht durchaus im Widerspruch zu einem aktuell sehr restriktiv regulierten Markt mit insbesondere sehr hohen (und anscheinenden immer noch weiter steigenden) Datenschutzstandards, die den technologischen Möglichkeiten der Vernetzung und der Datengenerierung, -analyse und -übertragung vielfach nicht gerecht werden.

Schon heute sind zahlreiche Smart Services und innovative Geschäftsmodelle in der Lebenswelt Wohnen vorzufinden, die sich in den kommenden Jahren weltweit voraussichtlich rasant weiterentwickeln und verbreiten werden. Die 
Potenziale liegen gerade in Deutschland jedoch bislang noch weitgehend brach. Dabei ist der Nutzen, den die Datensteuerung im intelligenten Zuhause liefert, zu großen Teilen bekannt, zugehörige Geschäftsmodelle entwickeln sich heute und Märkte werden international jetzt erschlossen und verteilt. Folglich sollte die Abwägung zwischen notwendigem Datenschutz und den möglichen Mehrwerten der datengetriebenen Geschäftsmodelle nicht einseitig geführt werden. Hier gilt es, die Balance unter Berücksichtigung aller Aspekte zu wahren, denn auch für Unternehmen stehen der Kundennutzen und das Kundeninteresse im Fokus ihrer Geschäftsmodelle (Bundesverband Digitale Wirtschaft 2018a, S. 3 f.).

\subsubsection{Rolle der Versicherer}

Datengetriebene Smart Services werden die Lebenswelt Wohnen in naher Zukunft nachhaltig verändern. Insbesondere gilt das für den Privatkundensektor, an den sich ein Großteil der Angebote richtet. Damit einher gehen das Eintreten neuer Marktteilnehmer, eine Neuverteilung bestehender Rollen sowie die Notwendigkeit, (branchenübergreifende) Kooperationen im Umfeld Wohnen einzugehen. In der jüngeren Vergangenheit ließen sich bereits einige Allianzen beobachten, und es zeigt sich eine langsam zunehmende Bereitschaft, Schnittstellen zur Verfügung zu stellen (Versicherungsbote 2018). Jedoch ist gerade der Smart Home-Markt nach wie vor sehr stark zersplittert. Zahlreiche Anbieter wie Hersteller von Haustechnik, Energielieferanten und Telekommunikationsunternehmen versuchen, den direkten Zugang zum Kunden zu gewinnen und sich mit ihren Lösungen am Markt zu positionieren.

Für die Kunden entsteht dadurch die Herausforderung zahlreicher unterschiedlicher Angebote, die teils überschneidende Services bieten und oft untereinander nicht kompatibel sind - insbesondere auch, was die Kommunikation der Geräte und den Datenaustausch untereinander angeht. Gerade durch die fehlende Kompatibilität der Geräte und Anwendungen untereinander ergeben sich Hemmnisse bei der Nutzung von Smart-Home-Technologien. Mehr und mehr wird deutlich, dass der Kunde kein Verständnis für separate und voneinander losgelöste Services hat, die einzeln installiert werden müssen und keine vollständige Lösung aufweisen (Deloitte 2018, S. 29). Vor diesem Hintergrund kristallisieren sich zunehmend Netzwerke heraus, die gemeinschaftlich an umfassenden, kundenzentrierten Smart Services arbeiten. Beispielhaft kann an dieser Stelle das Netzwerk ,Connected Living' genannt werden, an dem zahlreiche namhafte Industrie- und Softwareunternehmen, Dienstleister sowie Versicherer mitwirken 
(Connected Living 2018). Häufig dienen diese Zusammenschlüsse jedoch vorrangig dem Austausch und weniger der Entwicklung konkreter Lösungen, die umfassende Mehrwerte für die Bürger mit sich bringen. Bei der gemeinschaftlichen Entwicklung konkreter Leistungen überwiegen nach wie vor meist der Wunsch, das eigene Unternehmen und die damit verbundene Marke zu positionieren, sowie die Angst vor Konkurrenz und dem Verlust von Marktanteilen.

Mit den o. a. Entwicklungen in der Lebenswelt Wohnen wird sich auch das Geschäftsmodell der Versicherer verändern und werden neue Herausforderungen und Chancen entstehen. Mit den neuen Smart Services ändern sich zunächst die Kommunikationswege mit den Kunden und die Kontaktpunkte zu ihnen erheblich. Digitale Assistenten in den Wohnräumen schaffen eine völlig neue Form des Austauschs und bringen neue Touchpoints mit sich (Krohn 2018). Sie begleiten den Kunden in seinem privatesten Umfeld und schaffen dadurch eine genaue Kenntnis über seine Lebenssituation und Gewohnheiten. Mittels Datenanalyse können darüber hinaus Wünsche und Bedarfe der Kunden abgeleitet, passgenaue Leistungen individuell und bedarfsgerecht erstellt und über die intelligenten Interaktionsschnittstellen empfohlen und abgeschlossen werden. Das gilt auch für die Versicherungsprodukte rund ums Wohnen, die bei intelligenter Auswertung der Wohnausstattung und der damit verbundenen Sach- und Haftpflichtrisiken zielgenau, mit der treffenden Deckungshöhe und gesamthaft zugeschnitten angeboten werden können. Im Umkehrschluss kann ein smarter Helfer auch überflüssige oder nicht mehr notwendige Versicherungen aufdecken und kündigen. Außerdem kann er dem Kunden bei intelligenter Analyse den günstigsten Tarif vorschlagen und ihm bei neuen Angeboten einen Wechsel empfehlen. Ob es in Zukunft zudem noch derart zersplitterte Produktlandschaften geben wird, wie heute üblich, mit Wohngebäudeversicherungen zum ,Versicherungswert 1914', Hausratversicherungen nach dem ,Vollwertprinzip“ und Gewässerschadenhaftpflichtversicherungen für den Öltank im Vorgarten, die kein normaler Bürger versteht, kann hinterfragt werden.

Die Entwicklungen lassen auch eine weitere Konsolidierung des Versicherungsmarkts erwarten. Wenn in größeren Netzwerken künftig ganzheitliche Lösungen für das Smart Home des Kunden angeboten werden, könnte es ausreichen, daran jeweils nur einen Versicherer exklusiv zu beteiligen. Auch die personalen Versicherungsvermittler werden nicht wie bisher zum Einsatz kommen, wenn die digitalen Analysemöglichkeiten und die automatisierten Prozesse vermehrt zum Tragen kommen. Einerseits müssen sich die Versicherungsvertreter und -makler die digitalen Anwendungen selbst zunutze machen, andererseits werden die Entwicklungen aber auch zu einer Substitution ihrer bisherigen Arbeit führen. 
Außer für eine stärker kundenzentrierte Produktgestaltung können die in der Lebenswelt Wohnen gewonnen Daten unter Nutzung von Methoden des Data Mining und Künstlicher Intelligenz zur Prognose, Früherkennung und Prävention von Schäden verwendet werden, was ebenfalls erhebliche Auswirkungen auf das Geschäftsmodell der Versicherer mit sich bringt. Durch Sensoren in den Rohrleitungen werden Wasserschäden verhindert, smarte Schließ- und Überwachungssysteme beugen Einbrüchen vor, und auch Schäden durch Unwetter und Feuer können durch Wettersensoren, intelligente Rauchmelder u. ä. reduziert werden. Die bislang hohen Schadensummen, die besonders bei Gebäudeschäden im Zusammenhang mit Wasser und Feuer auftreten, können dadurch signifikant sinken (Munich Rr 2017). Kommt es dennoch zum Schadenfall, werden Schadenabwicklung und -regulierung durch eine intelligente Datenanalyse grundlegend vereinfacht, da der Versicherer für seine Aufgaben der Schadenprüfung viele relevante Daten in Echtzeit einsehen und verarbeiten kann. Mit der einhergehenden Ursachenanalyse könnten die lernenden Systeme zudem wiederkehrende Schäden immer besser vermeiden. Dadurch werden die Risiken in der Hausrat- und Wohngebäudeversicherung gemindert, und in der Folge sinkt auch schlicht die Bedeutung klassischer Versicherungsprodukte. Dadurch ist eine Neuausrichtung des Geschäftsmodells unausweichlich.

Allerdings kommen im Smart Home auch neue Risiken auf, die einer Absicherung bedürfen. So scheint es im Hinblick auf die wachsende Bedeutung von Daten- und Prozesssicherheit angezeigt, eventuelle Ausfälle des Smart Home-Systems oder Hackerangriffe abzusichern sowie bspw. bei möglichem Fehlalarm für die entstandenen Kosten aufzukommen. Um sich langfristig in der Lebenswelt Wohnen zu etablieren, ist die strategische Positionierung im Ökosystem Wohnen erforderlich. Von großer Bedeutung sind dabei branchenübergreifende Kooperationen und die Eingliederung in einem umfassenden Netzwerk - sei es als Orchestrator oder Zulieferer von Komponenten rund um Lösungen im Smart Home.

Einen Schritt hin zu strategischen Partnerschaften haben viele Versicherer in den letzten Monaten und Jahren bereits getan. Ein Beispiel dafür ist ein Produkt der ERGO, die in Kooperation mit der Deutschen Telekom einen SmartHome-Schutzbrief anbietet. Löst ein Magenta-Smart-Home-Gerät (Rauchmelder, Wassersensor, Alarmanlage) einen Alarm aus, wird der abwesende Bewohner über sein Smartphone informiert und über die ERGO werden entsprechende Notfallmaßnahmen eingeleitet. Der Versicherer ergänzt damit die technologischen Komponenten der Telekom um tatsächliche Hilfeleistungen im Schadenfall. Darüber hinaus übernimmt die ERGO im Rahmen der Kooperation auch die 
Schadendeckung und die Kosten für eine nachfolgende Bewachung bei eingeschlagenen Fenstern und Reparaturen bis zu einer Höhe von 3000 EUR.

Analog funktioniert die Zusammenarbeit zwischen dem Elektronikhersteller Gigaset und der AXA. Registriert die Alarmanlage von Gigaset einen Einbruch, wird der Kunde per Smartphone informiert und kann über das Überwachungssystem prüfen, ob es zu einem Einbruch gekommen ist. Im gegebenen Fall hat er die Möglichkeit, den angeschlossenen AXA-Sicherheitsdienst zu informieren, der sich um die weiteren erforderlichen Maßnahmen kümmert. Durch die konkrete Hilfeleistung durch den Versicherer wird der Service für den Kunden besser greifbar und Installationshemmnisse gegenüber einem Smart Home können abgebaut werden.

Einen ähnlichen Ansatz verfolgen Allianz und Panasonic mit ihrer Kooperation (Allianz Assist Versicherung), die u. a. einen kostenlosen Schlüsseldienst, eine Sicherheitskontrolle vor Ort und die Anbindung verschiedener Handwerkspartner organisieren. Auf der einen Seite zeigt sich, dass durch derartige Partnerschaften neue Absatzkanäle sowie über die Smart Home-Geräte zusätzliche Kontaktpunkte zum Kunden erschlossen werden können (Müller 2018). Auf der anderen Seite verdeutlichen die Beispiele, dass sich die Kooperationen aktuell nur auf bilaterale Partnerbeziehungen beschränken und mit verschiedenen Anbietern und Versicherern sehr ähnliche bis deckungsgleiche Services angeboten werden. Dadurch und mit den vorrangig singulären Smart Home-Produkten konnten bislang kaum Alleinstellungsmerkmale realisiert werden.

Damit eine umfassende Lösung für die Lebenswelt Wohnen angeboten werden kann, sind statt bilateraler Partnerschaften auf Produktebene übergreifende Kooperationen mit Akteuren aus mehreren relevanten Branchen erforderlich. In der Lebenswelt Wohnen überwiegt das Bedürfnis nach Sicherheit gegenüber dem Wunsch nach Innovationen und neuesten Technologien. Zudem wird ein großer Teil Privatsphäre berührt, wodurch Vertrauen und Zuverlässigkeit für viele Menschen eine wichtige Rolle bei der Wahl des Anbieters spielt. Dadurch entstehen für Versicherer Chancen der Positionierung, denn sie sind schon seit Langem dafür bekannt und akzeptiert, sensible Daten über ihre Kunden zu verarbeiten und sorgsam zu nutzen. Verschiedene Studien schreiben daher den Versicherern auch ein deutlich größeres Vertrauen beim Datenschutz zu als internationalen Großkonzernen wie Amazon oder Google. Dieses Potenzial könnte genutzt werden, um sich als ,Enabler' und Orchestrator eines umfassenden Netzwerks in Stellung zu bringen. Zudem haben Versicherer mit dem Ziel geringer Schäden ein den Kunden ähnliches Anliegen.

Das hohe Vertrauen der Kunden und die ähnlichen Interessen bei der Absicherung der eigenen vier Wände, verbunden mit den Potenzialen, die nach 
wie vor mit den Beratungs- und Betreuungskapazitäten einer breiten Vermittlerschaft verbunden sind, schaffen auch gute Voraussetzungen für den Kundenzugang. Damit könnte es einem Versicherer gelingen, Unternehmen aus anderen Branchen und deren Smart Services an das eigene Angebot anzukoppeln und das Netzwerk damit im Interesse des Kunden zu steuern. Der Versicherungsschutz muss dabei nicht im Mittelpunkt stehen. Vielmehr kann und sollte ggf. der Versicherer als umfassender Dienstleister auftreten, der sich im Interesse des Kunden um die Absicherung seiner Wohnumgebung kümmert, zugleich im Netzwerk Wohnkomfort und Ressourceneffizienz fördert und zudem bei Bedarf unkompliziert Versicherungsschutz und im Schadenfall auch Assistanceleistungen beisteuert (Müller 2018).

\subsection{Lebenswelt Gesundheit}

\subsubsection{Smart Services im Überblick}

Die Gesundheit zählt zu den höchsten Gütern der Menschen. Die Entwicklungen im Gesundheitssektor und der medizinische Fortschritt sind vor diesem Hintergrund in der Breite der Bevölkerung von großem Interesse. Ohne Übertreibung stellt die eigene Gesundheit per se eine ,Lebenswelt' für die Menschen dar. Die Entwicklung des Gesundheitswesens ist im Grunde so alt wie die Menschheit selbst, und die Technologieverwendung geht weit in die Vergangenheit zurück. Vor allem im letzten Jahrhundert sind die technischen Anwendungen im Gesundheitssektor immer schneller vorangeschritten. Im Zusammenspiel mit der zunehmend besser werdenden medizinischen Versorgung sowie der stärkeren Aufklärung über die verschiedenen Krankheitsrisiken und Präventionsmöglichkeiten werden die Menschen immer älter und bleiben länger gesund. Seit einiger Zeit hat sich zudem die ,Quantified Self'-Bewegung als bedeutender Trend entwickelt, der sich durch einen großen Teil verschiedener Altersstufen und durch breite Bevölkerungsgruppen zieht. Dieser Trend, zu dem neben der Selbstvermessung auch die Selbstoptimierung gehört, hat bedeutende Auswirkungen auf die Lebenswelt Gesundheit (Heyen 2016, S. 2). Viele der Verhaltensweisen und Einfluss nehmenden Faktoren, wie bspw. der Grad der sportlichen Betätigung oder die Ernährung, sind allerdings stark subjektiv geprägt und nicht ohne Weiteres kontrollierbar. Dafür rücken Alltagshelfer und Tracking-Systeme, die beim Aufzeichnen des eigenen Lebensstils helfen, immer stärker in den Fokus.

Einen starken Einfluss auf die Medizin, namentlich auf die Diagnostik und Therapie, haben Daten. Die Flut an Daten, die inzwischen gesammelt werden 
kann, führt zu besseren und genaueren Analysen und - insbesondere auch im Zusammenhang mit Künstlicher Intelligenz - zu besseren Vorhersagemöglichkeiten. Damit können nicht nur die Effizienz (schnellere Diagnose) und der Komfort (automatische Erhebung) medizinischer Leistungen gesteigert werden, sondern vor allem auch deren Qualität.

Eine Besonderheit der in der Lebenswelt Gesundheit erhobenen Daten liegt in dem hohen Grad der Sensibilität: Bei Gesundheitsdaten handelt es sich um personenbezogene Daten, die nach Art. 4 Nr. 15 der Europäischen Datenschutzgrundverordnung (EU-DSGVO, siehe hierzu auch Abschn. 2.2.3) besonders schutzbedürftig sind und daher einem ausgeprägten Datenschutz unterliegen. Gerade die sensiblen Personendaten sind aber in einem hohen Detaillierungsgrad notwendig, um die Bürger in der Lebenswelt Gesundheit unterstützen zu können und ihnen Mehrwerte zu bieten. Daher ergibt sich in besonderem Maße ein Spannungsfeld, das immer wieder stark in der gesellschaftspolitischen Diskussion steht.

Neben den Daten sind verschiedene Technologien, wie die Sensorik, die zunehmende Vernetzung im Rahmen des Internet of Things (IoT) oder die Künstliche Intelligenz (KI), essenzielle Bestandteile der Smart Services in der Lebenswelt Gesundheit. Dabei können die Smart Services wie folgt gegliedert werden:

\section{Datensammlung}

Ausgangspunkt und zugleich erstes mehrwertiges Ergebnis ist die Sammlung von Körpermessdaten. Zunehmend ermöglichen es technische Geräte, beispielsweise Smart Watches, smarte Waagen, intelligente Laufbänder oder andere Wearables, Körperdaten wie den Pulsschlag, die Körpertemperatur, die Sauerstoffsättigung, das Gewicht, die Muskelmasse, den Fett- und Wasseranteil zu messen. Angereichert werden können diese Informationen durch Daten wie die Blutgruppe, Allergien oder Vorerkrankungen. Zusätzlich können Arztberichte und Befunde die Auswertungen ergänzen, sodass die Zielsetzung und das Ergebnis ein Datenpool ist, der ein umfassendes Bild über den Gesundheitszustand eines Menschen liefert. Aus diesem Datenpool können unterschiedliche Akteure verschiedene Nutzen ziehen. So kann die messende Person selbst den eigenen Lebensstil analysieren und optimieren und mit anderen teilen - etwa um sich so in ihrer Peergroup zu vergleichen.

Im Zusammenspiel zwischen Patient und Arzt können mit einer qualifizierten Datenbasis und den Auswertungsmöglichkeiten Krankheiten und körperliche Beeinträchtigungen besser behandelt werden. Und die Forschung kann die Medizin durch Einbeziehung umfassenderer Datengrundlagen und verbessertem Zusammenhangswissen substanziell weiterentwickeln (Heyen 2016, S. 4 f.). 


\section{Diagnoseunterstützung}

Auf Basis der gesammelten Daten ergeben sich Smart Services, die Mehrwerte für die Diagnoseunterstützung bieten. Dabei gibt es verschiedene Anwendungsmöglichkeiten: So können Daten, die gewisse Anomalien signalisieren, eine automatische Meldung an den Nutzer mit dem Hinweis auslösen, einen bestimmten Arzt aufzusuchen. Digitale Fotos einer auffälligen Hautstelle können durch eine Analysesoftware über eine Datenbank gespielt und im Sinne einer Ersteinschätzung automatisch ausgewertet werden. Zusammen mit dem übermittelten Analyseergebnis kann gegebenenfalls zugleich ein geeigneter Facharzt zur näheren Begutachtung und anschließenden Therapie empfohlen werden. Auch mit Röntgenbildern, CT's und MRT's, ferner mit Blutwerten, EKG's und allen sonstigen möglichen Analysewerten lassen sich solche Datenbanken füllen und nutzen.

Unter Einbeziehung einer Künstlichen Intelligenz kann das System von den Ergebnissen nachfolgender Untersuchungen lernen und dadurch im Gleichschritt mit der Nutzungshäufigkeit verbessert werden. Systeme mit Künstlicher Intelligenz können über die Zeit immer ausgefeiltere Verknüpfungen von Gesundheitsdaten interpretieren und sich selbstlernend verbessern, d. h. Krankheitssymptome immer zuverlässiger automatisch erkennen und zielführende Therapien vorschlagen. Schon heute gibt es Künstliche Intelligenzen, die mittels bildgebender Verfahren, etwa der sog. Positronen-Emissions-Tomografie (PET), Demenzerkrankungen deutlich früher erkennen können als Ärzte. Das Ziel ist es, durch eine ausreichend große Zahl an Daten, die mithilfe vorangegangener und gespeicherter Untersuchungsergebnisse generiert wird, Befunde automatisch und damit sowohl schneller als auch präziser als von Menschen möglich zu erstellen (PwC 2017, S. 7 f.).

\section{Therapieunterstützung}

Die technischen Hilfsmittel zur Diagnoseunterstützung gehen Hand in Hand mit Mehrwerten, die Smart Services im Bereich des Monitorings von Heilungsverläufen oder im Rahmen der Unterstützung bei körperlichen Beeinträchtigungen im Alltag bieten. Nach Feststellung einer Erkrankung bieten digitale Anwendungen die Möglichkeit, den Therapieverlauf 24/7 für den Arzt abzubilden. Dieser erhält beispielsweise über die beim Patienten aufgezeichneten Daten Einblick in dessen Gesundheitszustand und empfängt zusätzlich eine Meldung, sobald ein bestimmter Wert abseits der Toleranz liegt. Mit einer dann erfolgten frühzeitigen Intervention kann der Heilungsverlauf schneller und effizienter weitergeführt werden. Wenn der Patient zusätzlich die Einnahme der Medikamente in die Anwendung einpflegt, kann der behandelnde Arzt schneller Ursachen für eine falsche Entwicklung der Genesung finden. Über cloud- 
basierte Kommunikationsplattformen kann das Zusammenspiel der verschiedenen Akteure, also Patient, Arzt, Krankenhaus, Pflegekraft, Krankenversicherer etc., erheblich verbessert werden und Doppel- und Mehrfachuntersuchungen können vermieden werden. Auch können das Internet of Things (IoT), also die Vernetzung z. B. von medizinischem Gerät, und der Austausch über Informations- und Kommunikationsplattformen wichtige Hilfestellungen für die Zusammenarbeit der verschiedenen Parteien geben.

\section{Ambient Assisted Living}

Ambient-Assisted-Living-(AAL)-Technologien können auch im Krankheitsfall oder bei körperlichen Beeinträchtigungen ein selbstbestimmtes Leben ermöglichen. AAL-Technologien beinhalten Produkte, Dienstleistungen und Konzepte, die die Lebensqualität vor allem älterer Menschen in allen Lebenslagen erhöhen sollen. Beispielsweise können Sensoren die Bewegungsabläufe in der Wohnumgebung messen und einen Notdienst alarmieren, wenn eine auffällige Bewegungsunterbrechung vorliegt. Viele solcher Systeme haben sich aufgrund der Scheu vor Technik, von Überwachungsängsten oder zu hohen Kosten noch nicht durchgesetzt. Einzig der Hausnotruf, bei dem ein Alarmknopf am Körper getragen wird, ist bereits weit verbreitet (Ambient Assisted Living Deutschland Acatech 2018).

\section{Transhumanismus}

Die Entwicklungen zeigen, dass es eine immer stärkere Verschmelzung von Mensch und Maschine gibt. Im Rahmen von Testversuchen werden bereits Technologien entwickelt, bei denen Chips oder Sensoren direkt unter die Haut des Menschen gelegt werden. Das enge Zusammenspiel zwischen der Digitaltechnik und dem Menschen kann zu einem ganzheitlichen Ansatz der Gesundheitsbegleitung führen, der einerseits die verfügbaren Datengrundlagen nochmals exponentiell ausweitet, andererseits die Potenziale für eine schnellere und zuverlässigere Diagnose und Therapie ebenso nochmals steigert.

Die verschiedenen Entwicklungen können eine Fülle von Problemen lösen, denen Patienten Ärzte, Pfleger und weitere Beteiligte gegenüberstehen. Die Zeitrestriktionen der Ärzte werden durch die Diagnosemöglichkeiten und Therapieempfehlungen mittels Technologie entschärft, die Behandlung der Patienten durch die Überwachung während der Genesung verbessert, umständliche Vorgehensweisen beispielsweise beim Arztwechsel durch die erleichterte Kommunikation zwischen den Parteien vereinfacht und effizientere und zielgerichtetere Pflegeleistungen auf Basis der AAL-Technologien werden ermöglicht. Immobilen Personen und Personen, die in einem Gebiet mit schlechter Arztabdeckung woh- 
nen, können durch die Technologie viele Wege sparen. In die Diagnose und Therapieempfehlung können Computersysteme eine große Zahl an Parametern einbeziehen und damit deutlich zuverlässigere und zudem noch schnellere Ergebnisse liefern.

Es zeigt sich, dass Smart Services in der Lebenswelt Gesundheit die Anwendungsfelder Prävention, Diagnostik, Therapie, Überwachung von Krankheitsverläufen, Pflegeunterstützung und nicht zuletzt Fitness/Freizeit/Lebensstil beinhalten. Diese Anwendungsfelder basieren auf der Erhebung von Daten, deren Auswertung zunehmend mit Künstlicher Intelligenz vorgenommen wird. Im Folgenden werden drei ausgewählte Geschäftsmodelle betrachtet, die bereits auf Märkten existieren und aus den zuvor beschriebenen Entwicklungen hervorgegangen sind. Dabei liegt der Fokus auf der Beschreibung und Würdigung der Nutzenversprechen, die mit diesen Geschäftsmodellen verbunden sind.

\subsubsection{Ausgewählte Geschäftsmodelle innerhalb der Smart Services}

\subsubsection{Apple Health}

\section{Beschreibung des Geschäftsmodells}

Nach den Anwendungsfeldern der Smart Services lässt sich das Geschäftsmodell von Apple Health in die Bereiche Datengenerierung, Prävention, Diagnostik, Therapie, Überwachung von Krankheitsverläufen und Fitness/Freizeit/Lebensstil einordnen. Apple Health ist eine Anwendung, mittels derer Gesundheitsdaten gesammelt, aufbereitet und ausgewertet werden können. Sie ist als App auf dem iPhone vorinstalliert und in die vier Gruppen ,Aktivität", ,Achtsamkeit', ,Schlaf‘ und ,Ernährung ' eingeteilt. Da das Smartphone selbst nur begrenzt Gesundheitsdaten erheben kann, sind zusätzliche Gadgets wie die Apple Watch oder kleine Adapter zur Messung der Blutwerte für eine umfassende Nutzung und Datenerhebung unabdingbar. Auch mit Geräten aus dem Haushalt, wie der digitalen Körperwaage oder einer intelligenten Zahnbürste, kann die Anwendung gekoppelt und mit Daten angereichert werden. Als kompatibles Hauptgerät von Apple misst die Apple Watch u. a. die Herzfrequenz. Bei besonders hohen oder niedrigen Frequenzen löst sie eine Warnung aus und gibt unter Umständen einen Hinweis, zum Arzt zu gehen (Stanford Medicine 2018).

Zum anderen ist in die Apple Watch ein sogenannter Beschleunigungs- und Gyrosensor eingebaut, der einen möglichen Sturz des Trägers erkennt. Nach einem Sturz kann mit einem Klick ein Notruf gesendet werden. Wenn der Gestürzte allerdings $60 \mathrm{~s}$ nicht reagiert, wird der Notruf automatisch ausgelöst. 
Über GPS wird den Rettungskräften bei Ausruf des Notfalls unmittelbar der Standort der gestürzten Person angezeigt, ohne dass sich der Nutzer zunächst orientieren muss. Durch die einfache und direkte Kommunikation über die Anwendung werden Fehler vermieden und die Effizienz gesteigert. Daneben erfasst die Apple Watch Freizeitaktivitäten und kann als eine Art Trainer im Sport agieren (Apple 2018a). Für Anwender der Freizeitfunktionen bietet Apple einige Gadgets zur Kopplung mit dem iPhone an. Dazu zählen u. a. ein smarter Fahrradhelm, ein smartes Fahrradsystem für Licht, Navigation, Diebstahlsicherung und Fitness Tracking, ein Springseil, ein Tennisschläger und weitere Geräte zur Messung von Körperdaten, wie Blutdruck- und Blutzuckermessgeräte und andere Überwachungssensoren (Apple 2018b).

Wegen der Notwendigkeit und des Angebots, weitere Geräte und Anwendungen zu nutzen, ist eine standardisierte Schnittstelle erforderlich, um den Datenaustausch zwischen den verschiedenen Geräten zu organisieren. Apple stellt mit ,CareKit‘ ein Open Source Software Framework zur Verfügung, mit dessen Hilfe Entwickler Apps generieren können, die beim Umgang mit Krankheiten helfen, indem sie die individuelle Behandlung unterstützen (bspw. Festhalten von Symptomen oder der Medikation). Ein ähnliches Software Framework stellt das ,ResearchKit" dar, das die medizinische Forschung adressiert und bei der Durchführung medizinischer Studien hilft, indem bspw. Teilnehmer registriert werden können (Apple 2018c). Damit werden zwei Funktionen angeboten, mit denen andere Entwickler Gesundheitsapps erstellen können, die auf die gesammelten Daten von Apple Health zugreifen und mit der Anwendung interagieren. Apple setzt also ausdrücklich den Anreiz, dass weitere Gesundheitsapps programmiert werden, und stellt gleichzeitig sicher, dass sie mit Apple Health, das weiterhin als Zentrum für alle Daten fungiert, kompatibel sind. Ebenfalls unterstützt Apple medizinische Studien, die mittels App Gesundheitsdaten auswerten (Apple 2018c).

Apple Health fungiert also als eine Art Zentrale, die über verschiedene Geräte mit Daten ,gefüttert` wird. Folgende Gesundheitsdaten können dabei u. a. ausgewertet werden:

- Atemfrequenz

- Blutdruck und -gruppe

- Body-Mass-Index

- Herzfrequenz

- Kalorienverbrauch

- Körpergewicht und -größe

- Körpertemperatur 
- Sauerstoffsättigung

- Schlafverhalten

- Schritte inkl. Treppenstufen bzw. Höhenmeter

- Stürze

Seit Einführung des Betriebssystems iOS12 ist für iPhone-Besitzer auch die elektronische Patientenakte ,Medisafe" verfügbar. Diese beinhaltet schon bislang die Möglichkeit der Eingabe von Medikamentierungen, verbunden mit der Funktion, vor Wechselwirkungen verschiedener Medikamente zu warnen. Ebenfalls wird ein Notfallpass angeboten, der einen besonderen Nutzen entfaltet: Im Sperrbildschirm werden relevante Gesundheitsdaten angezeigt, auf die im Notfall der Rettungssanitäter oder Notarzt direkt zugreifen kann, um dem Patienten zielgerichteter zu helfen. Die Möglichkeit, an einem Ort alle Daten zu sammeln, schafft die Voraussetzung, weitere Leistungen anzuknüpfen, die das Gesundheitssystem im Ganzen optimieren.

\section{Geschäftsmodellanalyse nach Canvas}

Apple Health adressiert als Kundensegment vorrangig die Endverbraucher und kann damit als B2C-Lösung klassifiziert werden. Die Funktionen von ,CareKit" und ,ResearchKit" richten sich allerdings eher an Unternehmen, wodurch auch eine B2B-Komponente vorliegt. Unter den Endkunden werden speziell iPhone-Besitzer adressiert, die technisch affin sind, Gesundheitsbewusstsein aufweisen und deshalb entweder ihre Werte beobachten, präventiv sportliche Aktivitäten ,tracken' oder den Umgang mit vorliegenden Krankheiten optimieren wollen. Für diese Zwecke und als weiteres Wertangebot bietet Apple Health eine Basis für eine umfassende Datensammlung, um damit einen Überblick über die eigene Lebensweise und Gesundheit bereitzustellen. Insbesondere liegen die Daten für ihre Nutzer nicht mehr nur an verschiedenen Stellen, wie z. B. Apps, getrennt vor, sondern sind in Apple Health zusammengefasst und damit komfortabel zugreifbar. Zudem bieten die Auswertungen der Daten eine Hilfestellung, Krankheiten frühzeitig zu erkennen und in die Behandlung einzutreten. Mithilfe des Notfallpasses gibt es eine weitere Absicherung bei Unfällen. Sozioökonomisch führen die Anwendungen zu einer möglichen Einsparung von Kosten, da durch die allgemeine Gesundheitsförderung und frühzeitigere Aufdeckung von Krankheiten weniger Arztbesuche notwendig sind und der Heilungsverlauf besser abgebildet und unterstützt werden kann, was ihn beschleunigt. Kundennutzen erzeugt auch die Transparenz, mit der der Nutzer über die eigene Lebensweise und seine Aktivitäten informiert wird. Dazu werden die Daten dem Nutzer 
auch grafisch aufbereitet. Ein weiteres Wertangebot liefert die Integration von Self-Service-Optionen. Neben der automatischen Datenerhebung sind weitere manuelle Eingaben, wie z. B. die Blutgruppe, vorliegende Allergien und Erkrankungen möglich. Durch die Zentralisierung der Daten in einer Anwendung steigt der Komfort für den Nutzer, und die Datenpflege wird erleichtert. Apple hat besonderen Wert auf eine intuitive App gelegt, sodass die Übersichtlichkeit auch beim angestrebten Ausbau der Datenbank und der Anwendungen nicht verloren geht. Der reduzierte Bedarf an Arztbesuchen, die Beschleunigung des Behandlungserfolgs und womöglich auch die Effizienzsteigerung im sportlichen Training führen zu Zeitersparnissen (Apple 2018d).

Als Kommunikations- und Vertriebskanäle bedient sich Apple Health der App sowie einer Homepage. Es besteht aber keine direkte Beziehung zwischen Unternehmen und Nutzer, vielmehr stellt die App Hilfsmittel zur Selbstbedienung zur Verfügung. Einnahmequellen von Apple Health werden indirekt durch den Verkauf der kompatiblen Geräte generiert, da die App selbst kostenlos ist. Die Kooperationspartner von Apple Health können vielfältig sein. Jeder App-Entwickler, der ,CareKit' oder ,ResearchKit' nutzt, aber auch Ärzte, Krankenhäuser, Apotheken und Pfleger können eingebunden werden (Apple 2018e). Zudem sind sowohl Kranken- als auch Unfall- und Berufsunfähigkeitsversicherer als mögliche Kooperationspartner prädestiniert - und damit Versicherungsanbieter aus allen Sparten des Versicherungsgeschäfts. Als Schlüsselressourcen stehen die Daten über den Nutzer und seine Gesundheit im Vordergrund. Des Weiteren wird medizinisches Know-how als intellektuelle Ressource eingesetzt, das zudem mit sich ständig weiterentwickelndem Zusammenhangswissen sowohl auf der Unternehmensseite als auch auf der Seite der Nutzer kontinuierlich gesteigert wird. Infolge der Datengenerierung, des Datenpooling und der Datenanalyse unter Verknüpfung vieler gesundheits- bzw. krankheitsrelevanter Parameter kann auch das medizinische Wissen insgesamt in der Gesellschaft erweitert werden - was wiederum ein besonderes Wertangebot darstellt.

\subsubsection{Digitale Krankenversicherer am Beispiel Oscar und Clover}

Ein digitaler Krankenversicherer nutzt für sein Geschäftsmodell im Kern neue Technologien, insbesondere für seine Produkte und Services (,Smart Services") sowie für die innerbetrieblichen und nach außen gerichteten Abläufe. Sie verwenden Daten aus Anwendungen wie beispielsweise Apple Health. Die Kommunikation erfolgt so weit wie möglich digital. Oscar und Clover, zwei Versicherer aus den USA, bieten auf diese Weise neue Möglichkeiten. 


\section{Beschreibung der Geschäftsmodelle}

Oscar ermöglicht den vollkommen digitalen und transparenten Abschluss einer Versicherungspolice. Dabei werden folgende Leistungen angeboten: Über ein Suchfeld in der Smartphone-Applikation kann der Versicherte seine Symptome eintragen, und er erhält daraufhin erste Hinweise über eine mögliche Erkrankung. Nach Anwählen einer Rückruf-Funktion meldet sich innerhalb kürzester Zeit ein Arzt oder eine Krankenschwester und berät den Kunden. Zusätzlich steht dem Anwender ein persönliches Concierge-Team zur Verfügung, das z. B. Arzttermine bzw. Rückrufe vereinbart, Krankenhausaufenthalte organisiert und Schreibarbeiten nach einem Krankenhausaufenthalt übernimmt. Außerdem unterstützt Oscar den Kunden dabei, einen gesünderen Lebensstil zu führen: Beispielsweise lässt das Unternehmen dem Versicherungsnehmer bei Erreichen einer vorgegebenen Schrittzahl, die über die App gemessen wird, einen Amazon-Gutschein zukommen (Hioscar 2018).

Clover sammelt Patienteninformationen zur datenbasierten Erstellung klinischer Profile der versicherten Personen. Dabei greift das Unternehmen auf ein Netzwerk aus Ärzten, Arzthelfern, Krankenhäusern und (Online-)Apotheken zurück, deren Daten von Clover zusammengeführt und ausgewertet werden. So nutzt Clover z. B. Informationen zur Krankheitshistorie eines Versicherten, um ihn bei der Genesung zielgerichtet unterstützen zu können. Wird einem Patienten ein Medikament verschrieben, das regelmäßig einzunehmen ist, wird dies von Clover getrackt: Wird keine diesbezügliche Abrechnung eingereicht, schaltet sich Clover ein, erinnert den Patienten und informiert über die ,Einnahmehinweise'. Falls Arzttermine versäumt wurden, erhält der Patient ggf. Hausbesuche von Mitgliedern des Clover-Health-Teams, dazu gehören z. B. Krankenschwestern, Krankenpfleger oder Sozialarbeiter. Mit den Präventionsmaßnahmen verfolgt Clover insgesamt das Ziel, den Gesundheitszustand der Versicherten zu verbessern, kostenintensive Gesundheitsleistungen zu vermeiden und damit die allgemeinen Aufwendungen für die Versorgung von Patienten zu senken. Weiterhin gehören zum Leistungs- und Serviceangebot ein digitaler Katalog, der eine Übersicht über die Medikamente gibt, die durch den Versicherungsschutz abgedeckt sind, ein digitaler Apotheken- und Ärztefinder sowie kostenfreie Zusatzleistungen, wie z. B. routinemäßige Augen- oder Zahnuntersuchungen (Cloverhealth 2018).

\section{Geschäftsmodellanalyse nach Canvas}

Beide Unternehmen bieten neue Smart Services im Bereich Gesundheit. Während das Wertangebot von Oscar den digitalen und transparenten Abschluss einer Versicherungspolice umfasst und den Versicherungsnehmer beim Erkennen und einer 
möglichen Behandlung unterstützt, konzentriert sich Clover auf die präventive Gesundheitsvorsorge, die auf einer softwarebasierten Datenanalyse beruht. Es können individuell zugeschnittene Informationen bereitgestellt, passende Ärzte gefunden, die Medikamenteneinnahme überprüft werden und vieles mehr.

Auch die individuellere Tarifierung auf Basis der erhobenen Daten stellt eine Neuheit dar. Die datenbasierte Risikoermittlung führt dazu, dass der Versicherte in der Krankenversicherung immer näher an seinem wirklichen Schadenerwartungswert policiert werden kann. Auch dadurch kann sich eine mittelbare Kostenreduktion ergeben. Beispielsweise wird das individuelle Krankheitsrisiko aufgrund der Anreizwirkungen für eine gesündere Lebensweise gesenkt, was sich für die Versicherten auch in niedrigeren Prämien widerspiegeln kann. U. a. durch die Hilfe bei der Suche nach einem passenden Arzt geben die digitalen Krankenversicherer ein Transparenzversprechen ab. Ebenfalls werben sie mit einer äußerst transparenten Kosten- und Leistungsdarstellung. Durch die digitalen Angebote sind eine ortsunabhängige Beratung und Betreuung möglich. Zusätzlich können viele Daten über den persönlichen Gesundheitszustand oder eine vorliegende Krankheit in einem geschützten persönlichen Datenfeld eingetragen werden, wodurch die Integration solcher Self-Service-Optionen stark zur Prozessbeschleunigung beiträgt.

Die Kundensegmente von Oscar und Clover liegen sowohl im B2B- als auch im B2C- und B2B2C-Bereich. Neben der eigenen Verbindung zu den Versicherten werden Ärzte, Krankenhäuser, Pfleger und weitere Gesundheitsdienstleister einbezogen. Als Versicherungskunden werden vor allem digital affine Menschen mit Gesundheitsbewusstsein angesprochen, die für finanzielle und medizinische Vorteile bereit sind, ihre Daten an den Versicherer zu geben. Da Clover zum Medicare-Advantage-Programm der USA gehört, stehen die Leistungen nur darin einbezogenen Personen offen. ${ }^{8}$ Im Jahr 2017 lag die Versichertenzahl von Oscar bei etwa 135.000, Clover zählte rund 25.000 Versicherte.

Als Kommunikations- und Vertriebskanäle werden diverse Social Media, Apps, Chatfunktionen und die Homepages genutzt. Weiterhin wird den Kunden der Kontakt über (Video-) Telefonie oder E-Mail angeboten, und in Blogs werden sie zudem regelmäßig mit Informationen versorgt. Auch in der Kundenbeziehung bietet dieses Geschäftsmodell einen weiten Spielraum. So ist von automatisierten Angeboten und Dienstleistungen über Selbstbedienungen bis hin zur persönlichen und auch individuell persönlichen Unterstützung, also mit

\footnotetext{
${ }^{8}$ Mindestalter 65 Jahre oder Menschen mit terminaler Nierenkrankheit oder bestimmten Behinderungen.
} 
immer gleichbleibendem Ansprechpartner, alles möglich. Primäre Einnahmequelle sind die Versicherungsprämien vonseiten der Kunden. Werden zusätzlich Kooperationen mit Ärzten oder anderen Gesundheitsdienstleistern vereinbart, ergeben sich weitere Einnahmen. So bezahlen etwa Dienstleister einen Preis dafür, in das Netzwerk einzutreten oder den Kunden in bestimmten Fällen vorgeschlagen zu werden. Die Schlüsselaktivität ist bei beiden Unternehmen die Bereitstellung von Versicherungsschutz, die Datenanalyse und die Pflege des Netzwerks. Die Mitglieder dieses Netzwerks, Ärzte, Krankenhäusern, Apotheken, Krankenschwestern, Pfleger und weitere Gesundheitsdienstleister, stellen die Schlüsselpartner der Unternehmen dar. Schlüsselressourcen sind die Kundenstammdaten und die Gesundheitsdaten der Versicherten. Auf Basis der gesammelten Daten aus den geschaffenen Netzwerkstrukturen und der effizienten digitalen Prozesse kann ein ganzheitliches Angebot an Smart Services und damit ein umfassendes Wertangebot zur Verfügung gestellt werden.

\subsubsection{Spannungsfeld Datennutzung und Datenschutz}

Wie gezeigt wurde, kann das Sammeln und die Nutzung von Daten in der Lebenswelt Gesundheit erhebliche Auswirkungen entfalten. So kann nicht nur die Gesundheit des Individuums verbessert, sondern auch eine Optimierung des gesamten Gesundheitswesens vorangetrieben werden. Systeme, die auf Basis von Daten Krankheiten in all ihren Parametern erfassen, künstliche Intelligenzen, die diese auswerten, und Assistenzsysteme, die Ärzte in ihrer täglichen Arbeit unterstützen, gehören zu den großen Chancen, die die Datennutzung mit sich bringt.

Dem gegenüber steht der berechtigte und notwendige Datenschutz, der im Gesundheitswesen auch spezifisch geregelt ist. In Art. 4 Nr. 15 der EU-DSGVO sind Gesundheitsdaten konkret berücksichtigt und abgegrenzt: „Gesundheitsdaten [sind] personenbezogene Daten, die sich auf die körperliche oder geistige Gesundheit einer natürlichen Person, einschließlich der Erbringung von Gesundheitsdienstleistungen, beziehen und aus denen Informationen über deren Gesundheitszustand hervorgehen" (Verordnung des Europäischen Parlaments und des Rates 2016/679 2018, Art. 4 Nr. 15). Nach § 22 des Bundesdatenschutzgesetzes (BDSG) und der EU-DSGVO gehören Gesundheitsdaten zur besonderen Kategorie personenbezogener Daten. Danach gilt für alle Gesundheitsdaten eine besondere Schutzbedürftigkeit. In diesem Zusammenhang besteht ein grundsätzliches Verbot der Verarbeitung von Gesundheitsdaten, das nur unter der Voraussetzung des Art. 9, 2a-j EU-DSGVO etwas aufgeweicht wird (Deutsche Gesellschaft für Qualität 2017). Das Verarbeitungsverbot gilt demnach nicht, wenn die betroffene Person für einen festgelegten Zweck zustimmt, dass ihre 
Daten genutzt und verwertet werden. Neben weiteren Erlaubnistatbeständen beziehen sich die Absätze $\mathrm{h}$ und i direkt auf Gesundheitsdaten. In Art. 9, $2 \mathrm{~h}$ EU-DSGVO (Verordnung des Europäischen Parlaments und des Rates 2016/679 2018) heißt es:

Die Verarbeitung ist für Zwecke der Gesundheitsvorsorge oder der Arbeitsmedizin, für die Beurteilung der Arbeitsfähigkeit des Beschäftigten, für die medizinische Diagnostik, die Versorgung oder Behandlung im Gesundheits- oder Sozialbereich oder für die Verwaltung von Systemen und Diensten im Gesundheits- oder Sozialbereich auf der Grundlage des Unionsrechts oder des Rechts eines Mitgliedstaats oder aufgrund eines Vertrags mit einem Angehörigen eines Gesundheitsberufs und vorbehaltlich der in Absatz 3 genannten Bedingungen und Garantien erforderlich.

Und in Art. 9, 2i EU-DSGVO (Verordnung des Europäischen Parlaments und des Rates 2016/679 2018) steht:

Die Verarbeitung ist aus Gründen des öffentlichen Interesses im Bereich der öffentlichen Gesundheit, wie dem Schutz vor schwerwiegenden grenzüberschreitenden Gesundheitsgefahren oder zur Gewährleistung hoher Qualitäts- und Sicherheitsstandards bei der Gesundheitsversorgung und bei Arzneimitteln und Medizinprodukten, auf der Grundlage des Unionsrechts oder des Rechts eines Mitgliedstaats, das angemessene und spezifische Maßnahmen zur Wahrung der Rechte und Frei-heiten der betroffenen Person, insbesondere des Berufsgeheimnisses, vorsieht, erforderlich.

Das hohe Niveau des Datenschutzes von Gesundheitsdaten macht die Durchführung einer Datenschutz-Folgeabschätzung notwendig, was zu einem erhöhten Aufwand in Einrichtungen des Gesundheitswesens führt und die Datennutzung erheblich erschwert (Deutsche Gesellschaft für Qualität 2017).

Für das Individuum hat der ausgeprägte Datenschutz den Schutz vor Missbrauch zum Ziel. Dazu gehört nicht nur der Schutz vor Hackerangriffen auf Gesundheitsdaten, die inzwischen häufig vorkommen (Ärztezeitung 2017). Auch die Weitergabe an Unternehmen, die die Gesundheitsdaten für verschiedene Zwecke verwenden können, wird kritisch gesehen. Die Sorge vor einer Datenweitergabe und vor Missbrauch ist bei den Bürgern jedoch deutlich geringer, wenn es um den Arzt geht, der die Daten verarbeitet, speichert und weitergibt. Laut der Studie ,Zukunft der Gesundheitsversorgung“ der pronovaBKK empfinden es $77 \%$ der Befragten als Vorteil, wenn Daten über Diagnosen, Behandlungen, Medikamente, Allergien oder ähnliches zentral gespeichert sind und sich Haus- und Fachärzte jederzeit einen umfassenden Überblick über den Gesundheitszustand eines Patienten verschaffen können. Auch das Durchführen von Video- oder Onlinebehandlungen sowie per Telefon würden $56 \%$ der 
Befragten begrüßen (Schlingensiepen 2017). Dies lässt sich damit begründen, dass die eigene Gesundheit für den Bürger ein sehr wertvolles Gut ist und bei besserer Hilfe, beispielsweise weil der Arzt einen guten Überblick über den Gesundheitszustand bekommt, die Risiken schwächer bewertet werden. Ebenso kann davon ausgegangen werden, dass eine Verbesserung der medizinischen Leistung im Interesse aller ist. So beschneidet der starke Datenschutz die Chance und damit den großen Mehrwert, der beispielsweise durch das Fortschreiten der Forschung auf Basis großer Datenmengen erreicht werden kann. Die Forschung und damit das Wissen in der Medizin, die Qualität der Behandlung und letztlich die Gesundheit des Einzelnen werden damit nachhaltig gestört. Dies gilt zumindest in Deutschland und Europa. Die datenbasierte Forschung auch und gerade im Gesundheitswesen wird beispielsweise in den USA und in China stärker vorangetrieben (PwC 2018), was letztlich auch zum komparativen Nachteil von Bürgern und Unternehmen hierzulande führen kann.

Es entsteht daher ein Spannungsfeld, das es nicht nur rechtlich, sondern auch politisch und vor allem im Dialog mit den Bürgern zu lösen gilt. Hierbei muss ein Abwägen zwischen dem Missbrauchsrisiko und der Datennutzung und -auswertung zur Verbesserung der Medizin erfolgen, wobei der Wille und die Wünsche der Bürger im Vordergrund stehen müssen. Der Datenschutz hat in diesem Zusammenhang eine wichtige Funktion, sollte aber möglichst nicht die Potenziale beschneiden, die neue technologische Möglichkeiten heute bieten. Für viele Anwendungen lassen sich die Hürden des Datenschutzes vermutlich durch ausdrückliche Einwilligungserklärungen der Betroffenen zur Datenerhebung, -speicherung, -analyse und -weitergabe überwinden. In Betracht kommen dabei auch ,Datenspenden" an Forschungseinrichtungen o. ä. Das gilt aber nicht für jede Situation. Beispielsweise ist die Voraussetzung zur Einwilligung bei einem Unfall, um gesundheitsrelevante Daten an einen Arzt oder ein Krankenhaus zu übermitteln, damit schneller eine adäquate Hilfe bestimmt und organisiert werden kann, vielfach nicht zweckmäßig oder unerfüllbar (Bass 2017, S. 34 f.). Allzu restriktive Datenschutzbestimmungen sind etwa in einer solchen Situation nicht hilfreich und sind vor allem nicht im Interesse derjenigen, die mit den Regelungen eigentlich geschützt werden sollen.

\subsubsection{Rolle der Versicherer}

Die Potenziale, die aufgrund der Menge an Daten und neuen Technologien in der Lebenswelt Gesundheit erwachsen, sind vielseitig und bringen viele Chancen, auch für die Versicherer. Wie in Abschn. 2.3.2.2 (Digitale Krankenversicherer am 
Beispiel Oscar und Clover) gezeigt wurde, liefern Daten die Grundlage zur Entwicklung neuer kundenorientierter Geschäftsmodelle. Dadurch werden allerdings auch ein Zutritt immer neuer Marktteilnehmer und die Bildung von Netzwerken (Ökosystemen) im Gesundheitssektor begünstigt, was die eigene Positionierung eines traditionellen Versicherers in diesem dynamischen Marktumfeld unabdingbar macht. Im Folgenden wird mit Blick auf das Gesundheitsthema auf traditionelle Krankenversicherer fokussiert. Sie werden heute unzweifelhaft gezwungen, sich die neuen technologischen Möglichkeiten und eine moderne Datenbasis zunutze zu machen, um ihre Aufgabe zu erfüllen, eine bestmögliche Gesundheitsversorgung für ihre Kunden hervorzubringen sowie auch ihre eigene Marktposition zu verteidigen und zu stärken. Dafür sind auch im digitalen Umfeld der Kontakt und der Zugang zum Kunden deutlich zu verbessern und dem Kunden gleichzeitig die relevanten Nutzenversprechen anzubieten. Umgekehrt ergibt sich ein Risikopotenzial, das darin liegt und sich konkretisiert, wenn Entwicklungen verkannt und sich daraus ergebende Chancen nicht genutzt werden.

Die Ausgangslage der Versicherer unter den neuen Entwicklungen ist als grundsätzlich gut einzuschätzen. Ihr Geschäftsmodell basiert seit jeher auf den Daten der Kunden und deren gesundheitlicher Situation. Zudem genießen sie beim Thema (Gesundheits-)Daten ein hohes Vertrauen (siehe dazu Nitschke 2018), was gerade in der sich digitalisierenden Datenwelt von hoher Relevanz ist. Herausforderungen der Versicherer bestehen jedoch in der Reaktions- und Innovationsgeschwindigkeit bei der Nutzung neuer Technologien und damit auch der Befriedigung der konkreten Kundenwünsche. Gerade in der inzwischen sehr dynamischen Lebenswelt Gesundheit ist eine hohe Reaktionsgeschwindigkeit essenziell, um stets die bestmögliche Versorgung zu bieten.

Insbesondere von Versicherungsunternehmen sind jedoch auch ethische und moralische Aspekte in besonderem Maße zu berücksichtigen: So muss geprüft werden, ob Personen, die weniger auf ihre Gesundheit achten, zukünftig etwa bei der Versicherungsprämie benachteiligt werden und inwieweit dies gesellschaftspolitisch vertretbar ist. Ebenfalls sollte der Druck auf die potenziellen Kunden und den Bestand an Versicherten geprüft werden, der mit einem indirekten Zwang einhergeht, die eigenen Daten zur Gesundheit und Lebensweise preiszugeben.

Dass umgekehrt mit einer möglichst umfangreichen Datenbasis allerdings im Endeffekt die allgemeine Gesundheit durch Förderung einer gesunden Lebensweise steigt, Diagnose und Therapie verbessert werden können, die Kosten für die Versicherer und Versicherten auf breiter Front sinken sowie Ärzte und das gesamte Gesundheitssystem entlastet werden, ist im gesellschaftlichen Diskurs ebenfalls zu berücksichtigen. In diesem Spannungsfeld müssen die Versicherer und die gesamte Versicherungswirtschaft ihren Weg finden. Schwierig wird dabei 
die Grenzziehung bei der Datenerhebung, -auswertung und -nutzung zwischen einerseits verhaltensgeprägten Gesundheitsdaten, die der Bürger selbst beeinflussen kann (z. B. durch seine Ernährung, Sport, Schlaf oder den Konsum von bzw. Verzicht auf Alkohol, Zigaretten und Drogen) und für die ihm daher auch eine Verantwortung zugeschrieben werden kann, und andererseits schicksalhaften Gesundheitsdaten (z. B. genetische Disposition).

Die weitere Entwicklung kann bei den Krankenversicherern eine grundlegende Änderung des Geschäftsmodells mit sich bringen. Hierbei ist nicht nur an die Möglichkeiten gedacht, Prämienrabatte für bestimmte Lebensweisen zu geben oder Anreize für Präventionsmaßnahmen zu setzen, um mit den damit sinkenden Behandlungskosten die Policen insgesamt günstiger anbieten zu können. Die aktuelle Marktsituation bietet vielmehr auch Ansätze für die Versicherer, sich in den entstehenden Ökosystemen Gesundheit als Orchestrator oder Zulieferer zu positionieren. Auf diese Weise können Kooperationspotenziale genutzt und die Zusammenarbeit mit Ärzten, Krankenhäusern, Pflegern und anderen Gesundheitsdienstleistern gestärkt werden. Möglicherweise haben dabei andere, schon digitaler aufgestellte Unternehmen und Branchen aufgrund einer ausgeprägteren Technologieaffinität, schlankeren Prozessen und einer höheren Agilität und Flexibilität derzeit einige Wettbewerbsvorteile. Umso wichtiger ist es für die Versicherer, sich auf ihre Kompetenzen zu konzentrieren und sich eine passende Rolle zu suchen.

Gerade in der Lebenswelt Gesundheit ist die Rolle des ,Enablers' für den Versicherer gut vorstellbar: Der Kundenzugang und das hohe Kundenvertrauen könnten genutzt werden, um sich stärker als eine Art vermittelnder Akteur zwischen den Parteien Patient, Arzt, Krankenhaus und Pflegedienstleister zu positionieren und somit für das Gesamtsystem die Schlüsselrolle als Orchestrator eines Netzwerks einzunehmen. Die Daten, die dadurch zusätzlich generiert werden können, sind eine wertvolle Ressource. Mit dieser Möglichkeit geht allerdings auch eine sehr große Verantwortung einher. Wenn alle Daten zentral bei einem Versicherer zusammenlaufen, ist er damit in einem hohen Maß im gesamten Ökosystem für die Datensicherheit der Kunden verantwortlich. Das vorher aufgeworfene Paradoxon, die Übersensibilisierung der Datensicherheit einerseits und der Wunsch nach besseren Gesundheitsleistungen andererseits, muss in dieser Rolle auch maßgeblich von den Versicherern gelöst werden.

In Betracht kommt für ein Versicherungsunternehmen natürlich auch die Rolle des Zulieferers in einem Ökosystem Gesundheit. Hier stellt sich die Frage, welche Kernkompetenzen darin eingebracht werden können. Typischerweise wird es sich dabei um die Risikotragung und die Finanzierung von Gesundheitsleistungen handeln. Eine gute Ausgangsposition haben die Versicherer auch mit ihren 
historischen Daten über die Gesundheit, Krankheitsverläufe und Behandlungsdaten ihrer Versicherten, die sie einbringen könnten - wobei natürlich der Datenschutz abermals eine zentrale Rolle spielt. Fallweise kommen weitere Kompetenzen infrage, wie z. B. die Kundenberatung und -betreuung, sowie die Übernahme von Backoffice-Funktionen und -Prozessen (Kalkulationsaufgaben, Abrechnungsleistungen, Datenverwaltung).

\subsection{Lebenswelt Mobilität}

\subsubsection{Smart Services im Überblick}

Die Digitalisierung des Automobils sowie die zunehmende Vernetzung von Verkehrsmitteln und der Verkehrsinfrastruktur werden in den nächsten Jahren den größten Wandel im Mobilitätssektor mit sich bringen. Der Kampf um den Kunden verschiebt sich dabei von der Fahrzeugebene auf die Ebene smarter Mobilitätsservices (Bratzel und Tellermann 2018, S. 3 f.). Künftig steht z. B. nicht mehr das Automobil selbst, sondern verschiedene Mobilitätsangebote und damit verbundene Dienstleistungen im Vordergrund. Zentral sind dabei a) die jederzeitige Verfügbarkeit von Mobilität, die durch On-Demand-Lösungen sichergestellt wird, b) die erhöhte Sicherheit im Straßenverkehr, die durch Kommunikation der einzelnen Fahrzeuge untereinander sowie den (Daten-)Austausch der Fahrzeuge mit ihrer Umwelt ermöglicht werden, und c) der Komfort für den Bürger, der durch verschiedene angebundene Services von seinen (Fahr-)Aufgaben entlastet wird und zahlreiche Mehrwertleistungen angeboten bekommt, wie z. B. Entertainment-Lösungen, die ihm das Fahrerlebnis so angenehm wie möglich machen. Der Kunde erhält individuelle ,Smart Mobility'-Dienstleistungen bedarfsgerecht je nach seinen persönlichen Präferenzen.

\section{Connected Car}

Laut einer PwC-Studie beträgt die jährliche Wachstumsrate vernetzter Fahrzeuge bis zum Jahr 2022 annähernd $25 \%$ (Ahlemann et al. 2016, S. 24 f.). In den nächsten Jahren werden daher nahezu alle neu produzierten Fahrzeuge mit dem Internet verbunden sein. Dadurch können Informationen und Daten mit anderen Verkehrsteilnehmern, Objekten in der Umgebung oder der Cloud ausgetauscht werden. Der Fahrer eines Connected Car wird über Umweltkonditionen, wie bspw. Verkehrsinformationen und Wetterdaten, informiert, erhält Hinweise von verbundenen Ampelsystemen oder Beschilderungen und tauscht Daten zur Position und Geschwindigkeit mit anderen Verkehrsteilnehmern aus. Dadurch können 
Wege optimiert und das Ziel schneller erreicht werden. Der Datenaustausch und die Kommunikation mit anderen Verkehrsteilnehmern helfen zudem, den Verkehr sicherer zu machen und Unfällen vorzubeugen. In diesem Zusammenhang wird oft von der Car-to-Car- bzw. der Car-to-Infrastructure-Kommunikation gesprochen (Bratzel und Tellermann 2018, S. 28).

Daneben ist die fahrzeuginterne Kommunikation ein weiterer Anwendungsfall im Rahmen des Connected Car. Durch im Fahrzeug verbaute Sensorik können potenzielle Mängel vom Fahrzeug selbst erkannt werden, und in den gegebenen Fällen erhält der Fahrer eine Benachrichtigung, die mit einer Handlungsempfehlung sowie einem Hinweis auf die nächste Werkstatt verknüpft sein kann. Dadurch können schadenvorbeugende Maßnahmen ergriffen und Reparatur- und Serviceleistungen optimiert werden (Chaudhuri 2018). Zunehmend gehören zu den fahrzeuginternen Daten auch Sensoren, die im Sitz oder im Lenkrad eingebaut sind und die Vitalparameter des Fahrers messen, um Müdigkeit und gesundheitliche Beeinträchtigungen frühzeitig zu erkennen.

Durch die Vernetzung mit der Umwelt und die Ausstattung mit einem Internetzugang wird ein Echtzeit-Daten-Austausch mit anderen Verkehrsteilnehmern und Geräten ermöglicht, und dem Fahrer stehen zahlreiche digitale Services zur Verfügung, die er während der Fahrt nutzen kann. Dazu gehören Navigationssysteme, Fahrassistenzsysteme, Entertainmentdienste und viele mehr. Darüber hinaus sind die gewonnenen Daten und Schnittstellen nicht nur zum Zweck der Verkehrssteuerung, sondern auch zur Strafverfolgung und für Versicherungsunternehmen nutzbar. Auch lassen sich gezielt personalisierte Werbung sowie standort- bzw. kontextspezifische Angebote einspielen. Bspw. können in der Umgebung befindliche Sonderaktionen, Events oder Spritpreise im Fahrzeugdisplay angezeigt werden.

Neben Bewegungsdaten entstehen im Zusammenhang mit dem Connected Car viele weitere Daten, die durch GPS, Kameras und Sensoren erfasst und verarbeitet werden und durch Nutzungs- und Verhaltensdaten des Fahrers angereichert werden können. Damit gibt der Fahrzeugnutzer einen umfangreichen Einblick in sein Fahrverhalten, seine Nutzungsgewohnheiten bis hin zu Konsumpräferenzen. Neben Fahrzeugdaten können Daten zum Fahrverhalten und -stil, zum Musikgeschmack, Kommunikationsdaten sowie Bewegungsprofile ausgelesen und verarbeitet werden (Bundesverband Digitale Wirtschaft 2018b, S. 2). Das Connected Car stellt die Voraussetzung für weitere Smart Services, wie bspw. das Car- oder Ridesharing und das autonome Fahren, dar. 


\section{Carsharing und Ridesharing}

Carsharing meint die gemeinschaftliche Nutzung von Fahrzeugen und deren spontane und kurzfristige Anmietung auf Grundlage einer Rahmenvereinbarung (Carsharinggesetz vom 5. Juli 2017 (BGBl. I S. 2230)). Damit wird Carsharing dem Trend der Sharing Economy und der On-Demand-Lösungen gerecht, indem je nach Bedarf spontan und ortsunabhängig Mobilität bereitgestellt wird.

Zur Nutzung von Carsharing stellt der Kunde seine Anmeldedaten und seinen Standort über eine App zur Verfügung. In der Folge kann er nahegelegene Fahrzeuge der Carsharing-Flotte ausfindig machen und per App reservieren. Die Fahrpreisabrechnung erfolgt nach in Anspruch genommener Leistung, meist minutengenau. Unterhaltskosten und andere Fixkosten sind in die Nutzungsgebühren eingepreist; dadurch zahlt der Fahrer nur, solange er den Service in Anspruch nimmt, und er muss keine gesonderten Sprit- oder Parkkosten aufwenden. Neben der Bereitstellung und Kurzzeit-Vermietung können dem Kunden ergänzende Dienstleistungen vor, während und nach der Fahrt angeboten werden (ADAC 2018). Beispielsweise kann der Kunde während des Supermarkteinkaufs kostenlos parken und dabei Rabatte im Lebensmittelmarkt erhalten. Im Rahmen des Carsharings werden personenbezogene Daten einschließlich der individuellen Bewegungsdaten über GPS, also u. a. Fahrstrecken, Fahrzeiten, Geschwindigkeiten, das Beschleunigungs- und Bremsverhalten, gesammelt, ferner Daten über das benutzte Fahrzeug. Neben Automobilen können im Rahmen des Bikesharings auch Fahrräder zur Kurzzeitmiete angeboten und damit gemeinschaftlich genutzt werden.

Ein anderer, dem Carsharing verwandter Smart Service ist das Ridesharing, bei dem ebenfalls die gemeinsame Nutzung eines Fahrzeugs im Mittelpunkt steht. Im Unterschied zum Carsharing erfolgt die Nutzung des Fahrzeugs jedoch zeitgleich durch verschiedene Personen (Fahrgemeinschaft). Die Organisation des Ridesharings erfolgt über eine Plattform, auf der anderen Nutzern das eigene Auto zur Mitfahrt angeboten oder selbst nach Mitfahrgelegenheiten gesucht werden kann (Randelhoff 2014). Außer im Rahmen von geplanten Fahrten (Bsp. Blablacar) ist auch das spontane Teilen von Fahrdienstleistern möglich (Bsp. Uber). Insbesondere beim spontanen Ridesharing sind Bewegungs- und Standortdaten Grundvoraussetzung für die Bereitstellung des Smart Services.

\section{Autonomes Fahren}

Die Fahrzeugtechnik befindet sich aktuell in der Entwicklungsstufe der Teilautomatisierung: Das teilautonome Fahrzeug kann vorausfahrenden Automobilen auf geraden Strecken folgen, dabei die Spur und den Abstand halten und bei Ver- 
kehrsstau Fahraufgaben auch vollständig übernehmen. In Zukunft ist der vollständig autonome Verkehr möglich, bei dem das Automobil in jeder Situation alle Fahrfunktionen übernimmt und die Fahrt zum automatisierten Shuttle Service wird. Die Fahrtzeit kann dann für andere Zwecke genutzt werden und das Fahrzeug verwandelt sich in einen Ort für neue Dienstleistungen (Eckstein et al. 2018, S. 4 f.).

Somit wird das Automobil nicht länger ein reines Fortbewegungsmittel sein, sondern es entwickelt sich zum mobilen Arbeitsplatz, Restaurant oder Hotelzimmer. Der Friseur kann seinen Kunden vor der Haustür abholen und ihn während des Haarschnitts zum nächsten Termin bringen. Das autonome Fahren erzeugt damit einen hohen Komfort und Zeitersparnisse. Ein autonomer Transportservice kann zudem im ländlichen Raum lebende Personen oder ältere Menschen mobiler machen. Ermöglicht wird das autonome Fahren durch eine Echtzeit-Datenanalyse, die mittels Sensoren und Aktoren erfasste Daten wie Lichtzeichen, Verkehrsschilder und andere Verkehrsteilnehmer verarbeitet und intelligent verknüpft.

\section{Verkehrsüberwachung}

Insbesondere in der VR China ist die digitale Verkehrsüberwachung mittels Datenaufzeichnung auf dem Vormarsch. Vernetzte Kameras und Messsysteme erfassen zahlreiche Daten im öffentlichen Raum, die sich mittels Bilderkennung und künstlicher Intelligenz automatisiert auswerten lassen und eine Reihe neuer Geschäftsmodelle hervorbringen (Wired 2018). So lassen sich bspw. Verkehrsflüsse mittels der gesammelten und analysierten Daten steuern: Vernetzte und mit Sensoren versehene Ampelanlagen erfassen Bild- und Audiodaten und optimieren mit einer smarten Signalsteuerung den Verkehrsfluss, um Staus und Unfälle zu vermeiden. Dies zeigt schon heute ein Feldversuch in der Metropole Peking: Auf der Strecke zwischen Flughafen und Innenstadt wurden alle Daten aus Verkehrsleitsystemen, Kameras und Fahrzeugen mittels künstlicher Intelligenz ausgewertet. Dadurch ließ sich das Verkehrsaufkommen in den darauffolgenden 45 min vorhersagen. Anschließend konnte der Verkehr so gesteuert werden, dass es auf dieser Verbindung keinen Stau mehr gab (Rohkamm 2018).

Automatisierte Verkehrsüberwachungssysteme sind damit in der Lage, das Verkehrschaos in der Stadt zu lösen. Die in der Verkehrsüberwachung aufgezeichneten Daten sind Fahrzeugdaten, Bewegungsdaten, Audio- und Bilddaten sowie personenbezogene Daten. Zudem dient die Verkehrsüberwachung dazu, regelwidriges Verhalten zu erkennen und automatisiert Schritte einzuleiten. Fahrzeuge und Personen, die sich verkehrswidrig verhalten, werden über das Nummernschild oder mittels Gesichtserkennung identifiziert, computergesteuert 
werden alle weiteren relevanten Daten zusammengetragen, und eine Ahndung des Regelverstoßes kann ohne menschliche Interaktion erfolgen. Dabei werden neben fest installierten Kamerasystemen mittlerweile auch Drohnen eingesetzt, die die mobile Verkehrsüberwachung noch flächendeckender ermöglichen.

\section{Connected Mobility}

Die bisher betrachteten Smart Services beruhen vorrangig auf dem Automobil als Mobilitätsträger. Daneben bestehen viele weitere Verkehrsmittel, deren Verknüpfungen weitere Smart Services hervorbringen. Anbieter wie bspw. moovel bündeln das Angebot verschiedener Mobilitätsdienstleister und Verkehrsformen auf einer Plattform und ermöglichen ihren Nutzern damit Informationen über den Zugang zum individuell besten Angebot (moovel 2018). Verknüpft werden im Rahmen solcher Connected-Mobility-Services typischerweise Car- und Ridesharing, öffentliche Verkehrsmittel, wie Busse und Züge, sowie Fahrdienstvermittlungen (Taxi). Der Nutzer gibt hierfür seinen aktuellen Standort oder einen anderen beliebigen Startpunkt sowie seinen Zielort ein und erhält Informationen über die verschiedenen Reisemöglichkeiten. Neben der Verbindung mit öffentlichen Verkehrsmitteln werden auch Leihfahrräder in der Nähe angezeigt, und zusätzlich hat der Nutzer die Möglichkeit, über die Plattform ein Taxi zu bestellen (Bratzel und Tellermann 2018, S. 30 f.). Der Kunde spart dadurch Zeit und kann bequem in nur einer App Reisemöglichkeiten vergleichen und, je nach Anbieter und bestehender Kooperation, die für ihn beste Option direkt über die Plattform buchen. Voraussetzung für das Angebot einer solchen Plattform ist ein umfassender Datenaustausch zwischen den einzelnen angebundenen Mobilitätsdienstleistern. Zugangs- und Kundeninformationsdaten werden nur einmal eingegeben (bzw. aus dem Google- oder Facebook-Konto abgerufen) und stehen für alle weiteren Dienste zur Verfügung.

\subsubsection{Ausgewählte Geschäftsmodelle innerhalb der Smart Services}

\subsubsection{ReachNow}

\section{Beschreibung des Geschäftsmodells}

Die Mobilitätsplattform ReachNow wurde 2016 von BMW ins Leben gerufen. Anfangs war ReachNow ausschließlich in Seattle verfügbar, das Angebot wurde dann aber auf andere Bundesstaaten ausgedehnt und ist seit 2017 auch in Chengdu, China, und damit außerhalb der USA nutzbar. ReachNow kombi- 
niert auf seiner per Smartphone erreichbaren Mobilitätsplattform verschiedene Mobilitätsangebote miteinander und stellt seinen Kunden damit eine flexible Lösung zur Verfügung, die von überall in Anspruch genommen werden kann. Der Kunde kann dabei zwischen unterschiedlichen Fortbewegungsmöglichkeiten entscheiden und die für ihn beste Option per Knopfdruck auswählen und buchen. Zwar sind darin auch Kooperationen mit dem öffentlichen Nahverkehr enthalten, im Vordergrund steht jedoch - sicher auch mit Blick auf den Mutterkonzern nach wie vor das Automobil.

ReachNow bietet drei unterschiedliche Services: Erstens enthält das Angebot die klassische Vermietung von Fahrzeugen. Dabei kann der Kunde per interaktiver App sehen, welche Fahrzeuge wo in der Nähe bereitstehen, das Wunschfahrzeug reservieren und für die geplante Fahrt freischalten. Alle Daten zum Standort, zur Verfügbarkeit sowie verschiedene weitere technische Informationen zum Fahrzeug werden zentral auf der Plattform gespeichert und können jederzeit von den Nutzern eingesehen werden. Das Angebot unterscheidet sich damit kaum von anderen Carsharing-Angeboten wie bspw. DriveNow oder Car2Go. Neben dem klassischen Carsharing bietet ReachNow aber zweitens mit dem Chauffeurdienst und drittens mit dem Reservierungs- und Zustellservice weitere Leistungen: Der Chauffeurdienst als Premiumfunktion der App ist ähnlich dem Angebot von Uber oder DiDi, d. h. auch hier werden dem Nutzer verfügbare Fahrzeuge inklusive Fahrer angezeigt, die per App ausgewählt werden können und den Nutzer auf Wunsch innerhalb weniger Minuten von seinem Standort abholen (Schaal 2016). Beide Parteien, also ReachNow-Kunde und der Fahrer, sind dabei miteinander verbunden: Kontakt- und Standortdaten werden geteilt, sodass der Standort des jeweils anderen jederzeit verfügbar ist. Der Kunde erhält in Echtzeit Informationen über die voraussichtliche Ankunftszeit des Fahrers. Das dritte Angebot, der Reservierungs- und Zustellservice, ist die On-Demand-Lösung der Plattform und erinnert eher an eine Bestellung bei Amazon als an die heute in Deutschland verfügbare Autovermietung: Auf Wunsch wird dem Kunden ein Fahrzeug nach Hause oder zu einem beliebigen anderen Ort gebracht. So kann der Nutzer bspw. angeben, am nächsten Tag zu einer bestimmten Uhrzeit ein bestimmtes Fahrzeug für seinen Weg ins Wochenende zu wünschen, das dann pünktlich vor seiner Haustür bereitsteht. Wird das Fahrzeug nicht weiter benötigt, kann es im ReachNow-Gebiet geparkt und damit auf sehr einfache Weise zurückgegeben werden. Die App informiert den Kunden auch über geeignete Parkflächen. Mittelfristig sollen BMW- und Mini-Eigentümer zudem in der Lage sein, ihren Privatwagen über ReachNow zu vermieten (ReachNow 2018).

Bei ReachNow kann der Kunde aus über 1000 Fahrzeugen von BMW und Mini auswählen. Von Automobilen mit klassischen Verbrennungsmotoren über 
Hybride bis hin zu Elektrofahrzeugen steht je nach Präferenz ein passendes Fahrzeug zur Verfügung. Dadurch erhält der Kunde Zugang zu aktuellen Fahrzeugmodellen verschiedener Klassen und Antriebsformen. Um dem Kunden weiteren Komfort zu bieten, haben die Fahrer die Möglichkeit, ihr Smartphone mit dem Entertainment-System des Automobils zu verbinden und so die eigene Musik abzuspielen sowie Messengerdienste und (Video-)Telefonie während der Fahrt in Anspruch zu nehmen. Der Nutzer kann somit die Fahrtzeit im fremden Mietwagen individuell gestalten, was ein hohes $\mathrm{Ma} ß$ an Convenience bedeutet. Dem Anbieter eröffnen sich erneut Anknüpfungspunkte zur Datensammlung sowie zur Anbindung weiterer Services.

Gesamtgesellschaftlich betrachtet wird jedes Fahrzeug, das unter einem Geschäftsmodell wie ReachNow betrieben wird, dank der geteilten Nutzung effizienter ausgelastet. In letzter Konsequenz sinken mit den komfortablen Nutzungsmöglichkeiten per App auch die Motivation und Notwendigkeit, überhaupt ein eigenes Fahrzeug zu besitzen, das die meiste Zeit ohnehin nur geparkt am StraBenrand oder in der Garage steht. Auf längere Sicht entstehen damit insbesondere in den Städten, in denen der Raum ohnehin begrenzt ist, viele wieder alternativ nutzbare Flächen, die bislang von massenhaft parkenden Fahrzeugen in Anspruch genommen werden.

\section{Geschäftsmodellanalyse nach dem Business Model Canvas}

Das Wertangebot von ReachNow liegt in der flexiblen und komfortablen Bereitstellung von Mobilität, die von den Kunden einfach und transparent genutzt werden kann. Ziel des Angebots ist es, individuelle Mobilität je nach Standort, Zielort, Zeitplanung, sonstigen Präferenzen und Budget zu ermöglichen. Neben kurzfristigen On-Demand-Lösungen bietet ReachNow auch Langzeitmieten an, die u. a. auch für Nutzer-Communities zur Verfügung stehen. So können bspw. Firmen oder Wohngemeinschaften ein Fahrzeug mieten und die Nutzung des Fahrzeugs untereinander aufteilen. Abgesehen von der Gruppen-Vermietung an Unternehmen (B2B2C) richtet sich das Angebot von ReachNow an das Kundensegment der Endverbraucher (B2C) (Schmidt-Lackner 2016). Zwar wird dabei keine spezielle Kundengruppe fokussiert, die Nutzer werden jedoch häufig in urbanen Gegenden zu finden sein und typischerweise kein eigenes Auto besitzen.

Der vorrangig genutzte Kanal ist die ReachNow-App. Zwar gibt es auch eine Webseite sowie Aktivitäten auf verschiedenen sozialen Median, die als Kommunikationskanäle dienen; die eigentliche Nutzung und damit der Distributionskanal des Wertangebots beschränkt sich allerdings auf die ReachNow-App. Die Kundenbeziehung ist durch einen hohen Grad an Automatisierung und Individualisierung gekennzeichnet. Es gibt kaum einen persön- 
lichen Kontakt, vielmehr enthält die ReachNow-App eine Reihe unterschiedlicher Self-Service-Optionen, über die der Kunde seine individuelle Dienstleistung buchen kann. Aktuell hat ReachNow über 100.000 Kunden, die mit dem ReachNow-Service bislang mehr als 1,2 Mio. km gefahren sind. Die Einnahmequellen von ReachNow setzen sich aus einmaligen Mitgliedsgebühren (15 US\$) und Nutzungsgebühren für die verschiedenen Dienstleistungen zusammen. Die Fahrzeugnutzung wird dem Kunden dabei pro Minute oder bei Langzeitmieten als Tagessatz in Rechnung gestellt.

Die Schlüsselaktivitäten zur Umsetzung des Geschäftsmodells bestehen im Betrieb der Plattform, der Bereitstellung und Verwaltung/Pflege der Fahrzeuge sowie der Koordination der angebundenen Partner. Neben der Bereitstellung verschiedener Mobilitätslösungen können auch die in der App erfassten Daten über das Nutzungsverhalten und die Fahrgewohnheiten genutzt werden, um dem Kunden weitere individualisierte Angebote zu stellen. Dies kann durch ReachNow selbst oder die angebundenen Schlüsselpartner erfolgen: Über verschiedene Kooperationen mit lokalen Regierungen, anderen Mobilitätsanbietern, Parkplatzbetreibern u. a. werden weitere Dienstleistungen angeschlossen, Rabatte gewährt und die Kundenbindung gefördert. Zudem investiert BMW in die städtische Infrastruktur, bspw. durch die Installation von Ladesäulen. Dadurch können wiederum neue Daten zu Ladezeiten und Tarifen gesammelt werden. Es zeigt sich, dass neben den Fahrzeugen die bereitgestellten und gewonnenen Kundendaten eine Schlüsselressource des Geschäftsmodells von ReachNow darstellen. Essenziell sind vor allem die Standort-Daten, die mittels GPS von jedem Fahrzeug sowie allen Nutzern jederzeit bereitstehen und abgerufen werden können. Dadurch ist es möglich, den Nutzern Fahrzeuge in der Nähe anzuzeigen und in Echtzeit nachzuverfolgen, wo sich der Nutzer und das Automobil gerade aufhalten. Neben den Standort- und Bewegungsdaten spielen die Nutzungs- und Verfügbarkeitsdaten eine wichtige Rolle. Jede Fahrzeugnutzung wird aufgezeichnet und alle Informationen über den Zustand des Wagens oder bspw. den Ladestatus von Elektrofahrzeugen sind jederzeit präsent. Auch dadurch kann die Ressource ,Fahrzeuge“ optimiert werden. Angereichert werden die Nutzungs- und Fahrverhaltensdaten der Kunden durch Daten, die im Rahmen der verschiedenen Kooperationen von ReachNow entstehen.

ReachNow erhält damit u. a. Informationen, wohin der Nutzer mit dem Auto fährt, wie schnell oder vorsichtig dies geschieht, wie oft er dabei telefoniert und welche Entertainment-Angebote genutzt werden. Dies ermöglicht es ReachNow wiederum, den Kunden passgenaue Produkte oder Dienstleistungen anzubieten womit die Rückkopplung zu den Wertangeboten vollzogen wird. 


\subsubsection{Tesla Software Version 9}

\section{Beschreibung des Geschäftsmodells}

Die Tesla Software Version 9 ist ein Fahrassistenz-System, das bei Model S, Model X und Model 3 der Automobile von Tesla eingesetzt wird und diese Fahrzeugmodelle zunehmend autonom steuert. Mit jedem weiteren Softwareupdate erhält das System neue Funktionen, die weitere Aufgaben des Fahrers übernehmen und damit dem Ziel des autonomen Fahrens näherkommen. Zwar trägt der Fahrer noch die Verantwortung und muss die Kontrolle über das Fahrzeug und die Verkehrslage behalten, viele Schritte werden aber bereits selbstständig durch das Fahrzeug durchgeführt.

Dazu gehören eine hindernisabhängige Fahrzeuggeschwindigkeit sowie die Kombination aus Lenkassistent und Spurwechselassistent, die den selbstständigen Spurwechsel des Fahrzeugs ermöglicht. Soll die Spur gewechselt werden, reicht ein einfaches Antippen des Blinkers. Mithilfe der am Fahrzeug angebrachten 360-Grad-Kameras beobachtet das Fahrzeug den Verkehr der benachbarten Spuren und wechselt im geeigneten Zeitpunkt auf die angrenzende Fahrbahn. Neben dem Spurwechsel ist damit auch die eigenständige Nutzung von Autobahnausfahrten möglich, wobei das Navigationssystem mittels der bereitgestellten Daten die optimale Route und die passende Ausfahrt selbst auswählt - die mit dem Lenk- und Spurwechselassistenten selbstständig befahren werden kann. Auch Anschlussstellen kann der Autopilot selbstständig ansteuern. Unterstützend wirkt dabei die ebenfalls neu verfügbare Totwinkelwarnung, mit der nun auch die Seite und das Heck des Autos kontrolliert werden und die vor Hindernissen im toten Winkel der Spur warnt, auf die eingeschert werden soll. Radfahrer oder andere Verkehrsteilnehmer werden vollautomatisch registriert und die Geschwindigkeit wird der Gefahrensituationen zur Unfallvermeidung angepasst.

Die Anpassung der Fahrtgeschwindigkeit an den Verkehr erfolgt selbstständig durch das Automobil, ohne dass ein manueller Eingriff erforderlich ist. Über acht außen am Fahrzeug befindliche Kameras zeichnet Tesla Videodaten über die Fahrzeugumgebung auf, die in Echtzeit im Bordcomputer verarbeitet werden. Dabei werden Informationen mit einer Reichweite von bis zu $250 \mathrm{~m}$ einbezogen. Die 360-Grad-Visualisierung des Fahrzeugs gewährleistet eine ganzheitliche Überwachung des Verkehrs und unterstützt die Reaktion auf die wahrgenommene Umgebung. Dafür werden durch die Software Version 9 neben Video- auch Ultraschall- und Radarsignaldaten analysiert, sodass auch durch dichten Nebel oder vorausfahrende Fahrzeuge hindurch Daten gesammelt werden können. Die Videodaten können aufgezeichnet und gespeichert werden (Tesla 2018a). 
Dadurch soll bspw. bei einem Unfall die Ursache leichter aufgedeckt werden können. Ergänzend werden weitere Funktionen, wie die Parkplatzsuche und das Einparken, autonom vom Fahrzeug durchgeführt. Allein die rechtliche Situation erfordert (noch) das Vorhandensein eines menschlichen Insassen mit Führerschein.

\section{Geschäftsmodellanalyse nach dem Business Model Canvas}

Bei der Geschäftsmodellanalyse nach dem Business Model Canvas muss zwischen dem Geschäftsmodell Tesla und der Softwareversion 9 unterschieden werden. Einige der Kernelemente des Business Model Canvas können für die Version 9 nicht angewandt werden; insoweit erstreckt sich dann die Analyse auf das Geschäftsmodell von Tesla im Allgemeinen.

Das Wertangebot der Tesla Version 9 liegt vornehmlich in der Unterstützung des Fahrers bei seinen Fahraufgaben. Durch teilautonomes Fahren wird der Fahrer entlastet, und er soll künftig in der Lage sein, sich während der Fahrt auf andere Interessen als das Steuern seines Fahrzeugs zu konzentrieren. Zudem sollen Unfälle vermieden und zu einer höheren Verkehrssicherheit beigetragen werden. Soweit dennoch Unfälle eintreten, sollen die Erfassungs- und Beweismöglichkeiten für die Verursachung verbessert werden (Tesla 2018b). Daneben ist der Prestige-Gewinn eines autonom gesteuerten Oberklassefahrzeugs für viele Kunden ein weiteres Kaufargument. Das angesprochene Kundensegment von Tesla-Fahrzeugen sind vorrangig Endkunden (B2B), ebenso sind aber auch B2B2C-Lösungen, bspw. über Leasing, möglich. Im B2B-Markt stellt das Fahrzeug besonders für innovative oder ökologisch orientierte Unternehmen eine Alternative zu herkömmlichen Oberklassedienstwagen dar. Als konkrete B2C-Zielgruppe stehen Nutzer höherer Einkommensklassen im Vordergrund, die technologischen Neuerungen gegenüber aufgeschlossen sind. Die Kundenbeziehung und -bindung werden über das Fahrzeug selbst gepflegt, indem regelmäßige Updates erscheinen und dadurch der Kontakt zu Tesla fortdauernd aufrechterhalten wird. Neben dem Fahrzeug selbst sind für Tesla die Flagship-Stores in ausgewählten Städten ein wichtiger Kanal, über den mit dem Kunden interagiert und Fahrzeuge abgesetzt werden. Des Weiteren werden unterschiedliche Foren und Communitys als Kommunikationskanäle genutzt, über die Tesla Kunden gewinnen und binden möchte.

Primäre Einnahmequelle ist der Verkauf von Fahrzeugen. Daneben werden auch durch die Nachrüstung bspw. mit Kamerapaketen sowie über Reparaturen und Wartungsarbeiten Einnahmen generiert. Eine zusätzliche, wenn auch nur kleine Einnahmequelle sind Subventionen der Forschungs- und Entwicklungsarbeit im Bereich autonomer Fortbewegung, die Tesla von unterschiedlichen 
Institutionen oder Förderprogrammen erhält. Daneben ermöglichen wiederum Kundendaten das Anbinden zusätzlicher Services, die eine zusätzliche Einnahmequelle darstellen können. Die Schlüsselaktivitäten, die zur Umsetzung des Geschäftsmodells notwendig sind, sind die Forschung und Entwicklung sowie die Produktion von Fahrzeugen. Die Software, die die autonome Fortbewegung steuert, bindet keine Kooperationen ein, weshalb es insofern keine Schlüsselpartner gibt. Als Grund hierfür nennt Tesla die Gefahr eines Verlusts von Know-how und erhöhter Fehleranfälligkeit bei verteilten Kompetenzen. Kooperationen und Partnerschaften gibt es nur bei Tesla-Komponenten, die keine autonomen Fahrfunktionen steuern (bspw. Partnerschaften im Bereich der Ladestationen und der Batterieentwicklung). Damit umfangreichere Daten generiert werden können, fallen Kosten für die Softwareentwicklung an, und die Fahrzeuge müssen entsprechend aufgerüstet und mit Kamerasystemen und Sensoren ausgestattet sein. Außerdem verursachen die Pflege der IT-Systemlandschaft und die Rechenzentren, die von Tesla eingesetzt werden, hohe Kosten. $\mathrm{Zu}$ den Schlüsselressourcen von Tesla zählen zunächst Rohstoffe und Personal zur Entwicklung und Produktion von Fahrzeugen. Die Schlüsselressource des Systems, das das autonome Fahren ermöglicht, ist die Tesla-Software, die wiederum nur mit den Fahrzeug- und Umgebungsdaten funktioniert - die also letztlich maßgebliche Schlüsselressourcen für das Wertangebot von Tesla darstellen.

\subsubsection{Spannungsfeld Datennutzung und Datenschutz}

Datengetriebene Services in der Lebenswelt Mobilität bieten nicht nur eine höhere Sicherheit im Straßenverkehr, ${ }^{9}$ sie ermöglichen auch eine neue Form der Flexibilität und Bequemlichkeit, bei der zahlreiche unterschiedliche Mobilitätsformen jederzeit und für jeden bereitstehen. Darüber hinaus enthalten Smart Services in der Lebenswelt Mobilität zahlreiche Komponenten, die den Komfort der Bürger erhöhen, wenn sie sich im öffentlichen Raum bewegen. Beispielsweise können bequem verschiedene Transportmittel für eine Reise nach den Präferenzen der Nutzer aufeinander abgestimmt und miteinander verknüpft

\footnotetext{
${ }^{9}$ Nach wie vor ist der häufigste Grund für Unfälle das Abkommen des Fahrzeugs von der Fahrbahn, oft durch Unachtsamkeit. Durch intelligente Assistenzsysteme, die den Fahrer durch die Auswertung von Videodaten bspw. beim Halten der Spur unterstützen (Spurhaltesysteme), können Unfälle vermieden und die Sicherheit im Straßenverkehr erhöht werden.
} 
werden, bei Autofahrten wird die Parkplatzsuche erleichtert oder obsolet, und die Bezahlung erfolgt einfach per App (Goodwall et al. 2017, S. 114 f.). Ermöglicht wird dies durch das Erkennen und Auswerten der Bedürfnisse und typischen Verhaltensweisen verschiedener Straßenverkehrsteilnehmer, die Bereitstellung verschiedener Mobilitätsformen, ergänzender Services sowie Bezahlsysteme.

Im Hinblick auf die Datenerhebung ergibt sich in der Lebenswelt Mobilität eine Besonderheit: Zum einen geht es um die eigenen Daten, die bspw. ermöglichen, dass jederzeit und von überall auf verschiedene Dienste zugegriffen werden kann (Bsp. Apple CarPlay ${ }^{10}$ ) oder auf Basis persönlicher (Standort-).

Daten nutzerorientierte Empfehlungen abgegeben werden (Bsp. Google Maps). Daneben ist im Bereich der Mobilität auch die Interaktion mit anderen Personen und Objekten erforderlich (z. B. bei Navigationsservices und beim autonomen Fahren), d. h. es müssen auch Daten Dritter verfügbar sein und verarbeitet werden, um smarte Services zum Nutzen aller zu generieren. Dies sei noch etwas näher erläutert:

Moderne Fahrzeugmodelle sammeln mit einer großen Menge eingebauter Sensoren schon heute Daten über die gefahrene Geschwindigkeit, das Beschleunigungs-/Brems- und Kurvenverhalten, die gefahrenen Gänge und Drehzahlen, die Abstimmung des Fahrgestells (z. B. Öko, Komfort, Sport), womöglich auch den eingestellten Radiosender und natürlich die Ausgangs- und Zielorte sowie Routen. Dadurch können relevante Informationen zum Fahrzeug und dessen Nutzung ausgewertet werden. Zugleich werden Verhaltensdaten der Fahrer erfasst, die dazu geeignet sind, Bewegungsprofile zu erstellen, das Fahrverhalten zu analysieren, um auf dieser Grundlage Smart Services anzubieten. So ermöglichen Standort- und Bewegungsdaten überhaupt erst die Lokalisierung einer Person und/oder ihres Fahrzeugs. Dadurch können geeignete Angebote in der Nähe ausfindig gemacht werden (bspw. Tankstellen, Parkplätze, Restaurants, Sehenswürdigkeiten) und in Kombination mit den Standort- und Bewegungsdaten anderer Personen und Fahrzeuge eine Einordnung in das Umfeld sowie ein Gesamtbild der Verkehrssituation erzeugt werden (Goodwall et al. 2017, S. 119 f.). Solcherart Daten werden bspw. von Navigationsgeräten genutzt, um das Verkehrsaufkommen $\mathrm{zu}$ analysieren und $\mathrm{zu}$ prognostizieren sowie optimale Verkehrsrouten $\mathrm{zu}$ bestimmen - und damit zu einer effizienten Verkehrssteuerung (weniger Staus, weniger Unfälle) beizutragen. Zudem ermöglicht der

\footnotetext{
${ }^{10}$ Apple CarPlay ermöglicht die Nutzung aller möglichen Dienste (persönliche Musik hören, navigieren, Nachrichten schreiben) im Auto.
} 
gesamthafte Verkehrsüberblick, akute Gefahrensituationen zu erkennen (z. B. ein brennendes Fahrzeug hinter einer Kurve auf der Landstraße), um daraus Handlungsempfehlungen abzuleiten und die Sicherheit im Straßenverkehr zu erhöhen.

Wie in anderen Lebenswelten auch, ist allerdings der Datenschutz ein restringierender Faktor, der im Mobilitätssektor verschiedene Aufgaben erfüllen soll. Dazu gehören der Schutz der informationellen Selbstbestimmung sowie der Privatsphäre. Soweit eine Zuordnung von Daten zum polizeilichen Kennzeichen oder der Fahrgestellnummer - bzw. letztlich zum Fahrer oder Halter selbst erfolgt oder ermöglicht wird, handelt es sich um personenbezogene Mobilitätsdaten, die der Anwendung der EU-DSGVO unterliegen. Hinzu kommt, dass die Datenaufzeichnung nicht - wie in der Lebenswelt Wohnen vorrangig der Fall freiwillig und im privaten, geschlossenen Umfeld erfolgt, sondern im öffentlichen Raum stattfindet und dadurch der individuelle Bürger wenig bis keine Chancen hat, sich dieser zu entziehen. Dadurch besteht die Gefahr einer Verwendung, die nicht nur Smart Services unterstützt oder vor Verkehrsdelikten und Verkehrsunfällen schützt, sondern auch eine umfassende Überwachung der Bürger ermöglicht. Sowohl Regelungen der Datenhoheit als auch des Datenschutzes sind daher auch im öffentlichen Interesse. Die vertrauensvolle Preisgabe von Informationen durch die Nutzer und ein adäquater Schutzstandard sind daher essenziell für die Entwicklung und die Nutzung smarter Mobilitätsservices.

Damit ist nicht nur der Schutz vor Datenmissbrauch gemeint - wie z. B. dem Missbrauch von Bewegungsprofilen oder einer unter Umständen unfairen Bepreisung von Smart Services in Notlagen (Gründinger 2018, S. 3). Auch durch Hackerangriffe auf sensible Schnittstellen zwischen der Software des Fahrzeugs und anderen vernetzten Verkehrsteilnehmern und mobilitätsrelevanten Infrastruktureinrichtungen (Ampeln, Bahnübergänge, Zugbrücken, Tunnelschranken) können erhebliche Schäden angerichtet werden. Außerdem kann das Connected Car über ein Botnet ${ }^{11}$ angegriffen werden. Damit bestehen erhebliche Sicherheitsrisiken, dass Hacker die Kontrolle über die vernetzten Fahrzeuge erlangen oder zumindest die Fahrer die Kontrolle über ihre Automobile verlieren. Insbesondere während der Fahrt hätte dies fatale Auswirkungen auf die Verkehrssicherheit: Unfälle könnten herbeigeführt und der Verkehr könnte vollständig gestört oder lahmgelegt werden.

\footnotetext{
${ }^{11}$ Ein Botnet oder Botnetz ist eine Gruppe automatisierter Schadprogramme, sogenannter Bots. Die Bots laufen auf vernetzten Rechnern, deren Daten und Netzwerkanbindung ohne Einverständnis des Datensouveräns zur Verfügung stehen.
} 
Wer den Zugang und die Kontrolle über die gesammelten Mobilitätsdaten erhält, wird entscheidend an der Gestaltung und dem Angebot künftiger Fortbewegungsmöglichkeiten mitwirken. Neben Automobilherstellern sind es zunehmend auch neue Wettbewerber, wie Tech-Unternehmen oder Softwareentwickler, die über ihre Plattformen und Apps insbesondere im Bereich Connected Car Einblicke in Fahrzeug- und Verhaltensdaten der Nutzer erhalten (Becker und Pawelke 2015). Die Frage, wem welche Daten gehören, ist dabei oft noch nicht abschließend beantwortet.

Allerdings ist auch eine zu enge Regulierung hinderlich. So warnen z. B. Automobilhersteller in Deutschland und Europa vor zu starken gesetzlichen Einschränkungen und befürchten, dass die Europäische Union im Gegensatz zu Ländern wie China oder den USA zu enge Grenzen für die Nutzung der Mobilitätsdaten setzt. Damit werden nicht nur Smart Services behindert, sondern auch die unternehmerische Wettbewerbsfähigkeit aufs Spiel gesetzt. Unterschiedliche länderspezifische Regelungen und divergierende Rechtsprechungen betreffen z. B. das autonome Fahren: So ist der Fahrassistent von Tesla bislang nur in den USA und (noch) nicht in der EU verfügbar. ${ }^{12}$ Aufgrund teilweise deutlich großzügigerer Datenschutzrichtlinien haben Unternehmen außerhalb Europas insgesamt weit größere Spielräume, datenbasierte Mobilitätsdienstleistungen zu erforschen und anzuwenden.

Innovative Smart Services, wie das autonome Fahren und Ride Sharing, werden in jedem Fall kommen und sich weiter ausbreiten - ob die Angebote in den USA, China oder Europa entwickelt werden und in welchen Ländern die Wertschöpfung erfolgt, ist dafür nicht maßgeblich. Und sie werden mittelfristig auch ganze Branchen und Arbeitswelten verändern. Traditionelle Arbeitsplätze werden in Gefahr geraten, die durch eine intelligente und vernetzte Datenverarbeitung sowie datengesteuerte Automatisierung überflüssig werden (Oliver Wyman 2018). Das beste Beispiel ist der Taxifahrer, der zuerst durch Uber und Didi und in nicht allzu ferner Zukunft durch Künstliche Intelligenz ersetzt wird. Automobile werden zu Dienstleistungsorten, in die während der Fahrt Nachrichten übermittelt, in denen bildschirmgestützte Beratungsgespräche geführt und die Haare geschnitten werden können. Autonom gelenkte Fahrzeuge von Flottenanbietern werden zunehmend Privatwagen ersetzen, d. h. die Nachfrage nach einem eigenen Fahrzeug sinkt (Bratzel und Tellermann 2018, S. 13).

\footnotetext{
${ }^{12}$ In den USA hat der Kongress bereits ein Gesetz für autonom fahrende Fahrzeuge erlassen, in der EU sind viele Punkte noch offen.
} 
Dies wird auch Druck auf politische Rahmenbedingungen erzeugen. Diesbezüglich steht Deutschland, ein Land, dessen Wirtschaft besonders stark vom Automobil abhängt (Herstellung, Finanzierung, Versicherung), im besonderen Fokus, Lösungsvorschläge anzubieten. Und dies wiederum erfordert auch die Kooperation von Politik und Gesetzgebung mit den betroffenen Branchen und Verbraucherschutzeinrichtungen, ein ausgewogenes Verhältnis zwischen den Möglichkeiten der Datennutzung und den Notwendigkeiten des Datenschutzes rund um smarte Mobilitätsdienstleistungen herzustellen.

\subsubsection{Rolle der Versicherer}

Die Lebenswelt Mobilität ist seit jeher von einer großer Veränderungsdynamik geprägt. Die Fortbewegung spielt für die Menschen immer eine große Rolle, und die Wichtigkeit einer schnellen und komfortablen Fortbewegung von A nach B gewinnt in einer zunehmend globalisierten Welt fortwährend an Bedeutung: Mehr als eine Billion Euro investieren private EU-Haushalte pro Jahr, um mobil zu sein. In Deutschland fließt jeder siebte Euro in Mobilitätsangebote, und es entstehen immer neue Mobilitätsformen (ADAC 2017, S. 6). Der Mobilitätssektor ist daher von einer hohen Aufmerksamkeit geprägt, die mit einem intensiven Wettbewerb einhergeht.

Nach wie vor ist das Automobil das wichtigste Verkehrsmittel, jedoch verändert sich seine Bedeutung rasant und es vollzieht sich eine Entwicklung, bei der das Auto immer weniger Symbol der Freiheit und des Status ist. Nicht nur aufgrund überfüllter Straßen verliert das eigene Fahrzeug langsam an Attraktivität und Bedeutung und wird zunehmend durch neue Mobilitätsangebote abgelöst. Auch der Einstellungswechsel weg vom ,Eigentum‘ hin zur ,Nutzung', der mit den aufkommenden Sharing-Konzepten einhergeht, verschiebt die Gewichtungen. Mit der Etablierung verschiedener Smart Services verlegt sich der Fokus von der Ingenieurstechnik und der Hardware auf agile und komfortable Lösungen. Der veränderte Markt, der bisher von Automobilherstellern und Verkehrsbetrieben geprägt war, bringt zusätzlich neue Wettbewerber mit sich. Mit den beschriebenen Smart Services in der Lebenswelt Mobilität verändert sich auch das Geschäftsmodell der Versicherer.

Die zunehmende Vernetzung im Zusammenhang mit dem Connected Car bringt für die Assekuranz sowohl Potenziale als auch Herausforderungen mit sich. Durch die mit zahlreichen Sensoren ausgestatteten Automobile können Unfälle verhindert, Unfallhergänge rekonstruiert und eingetretene Schäden durch das Fahrzeug selbst gemeldet, automatisiert erfasst und begutachtet wer- 
den. Die Schadensabwicklung kann damit deutlich schneller und reibungsloser erfolgen, wozu auch neue Technologien wie Chatbots und - in der Logistik Drohnen beitragen. Damit werden die Prozesse der Versicherer effizienter, und auch für die Kunden werden die Abläufe deutlich vereinfacht und komfortabler gestaltet. Die Abrechnung erfolgt objektiver und damit grundsätzlich fairer, Versicherungsbetrug wird eingedämmt, und nicht nur der Verwaltungsaufwand für die Versicherer sinkt durch die datengetriebenen Services, vermutlich reduziert sich auch das Streitpotenzial bei der Schadenregulierung, was im Ergebnis auch Gelassenheit und Zufriedenheit auf der Kundenseite erhöhen könnten. Jedoch ist der Zugang zu den relevanten Daten für die Versicherer erschwert. Die Daten werden von Systemen generiert, die im Fahrzeug verbaut sind, oder von Kommunikationsmedien, wie dem Smartphone. Damit sind es zunächst die Autohersteller, Telekommunikationsanbieter bzw. Tech-Konzerne, die mit dem Connected Car wertvolle Mobilitätsinformationen einschließlich Informationen über das Fahrverhalten bekommen.

Schon für die Entwicklung und Etablierung von Telematik-Tarifen benötigt aber auch der Versicherer solche Verhaltensdaten, zu denen auch Zugang gewonnen werden kann, wenn der Nutzer die Informationen ausdrücklich mit dem Versicherer teilt. Während Automobilhersteller oder Tech-Unternehmen aber den Vorteil haben, anhand der ausgewerteten Daten tatsächliche Mehrwertleistungen und neue Smart Services anbieten zu können, sind die Anwendungen der Versicherer bislang noch weitestgehend auf die Kalkulation der versicherungstechnisch richtigen Prämienhöhe und damit auf eine Prämiendifferenzierung reduziert, die naturgemäß nur den ,guten Risiken“ nützt, also den Risiken mit unterdurchschnittlichen Schadenerwartungswerten. Nach den Regeln der Statistik ist aber immer auch rund die Hälfte der Risiken mit überdurchschnittlichen Schadenerwartungswerten unterwegs, für die Telematik-Tarife eher nachteilig wären. Abgesehen davon, dass allerdings die Korrelationen zwischen dem Fahrverhalten und der Schadenträchtigkeit noch keineswegs hinreichend sicher ermittelt sind und deshalb Telematik-Tarife noch in Kinderschuhen eines Versuchsstadiums stecken, sind damit die unmittelbaren Nutzenpotenziale auf bestimmte Kundengruppen eingeschränkt. Nur wenn es den Versicherern gelingt, über gesellschaftlich hoch akzeptierte Anreiz- und Sanktionsmechanismen die Motivation für ein ,besseres ' Fahrverhalten auf breiter Front zu erhöhen, ist damit ein Nutzen für die gesamte Kundschaft in der Kfz-Versicherung generierbar - bis hin zu einem Beitrag für den gesamtgesellschaftlichen Nutzen einer reduzierten Zahl von Unfällen mit Sachschäden, Verletzten und Toten. Insgesamt liegt die große Herausforderung der Versicherungswirtschaft darin, mit den neu gewonnen Mobilitätsdaten echte Mehrwerte für die Kunden und Nutzenpotenziale auch 
jenseits einer für einzelne Zielgruppen günstigeren Tarifierung und Prämieneinstufung zu erzeugen.

Die Rolle der Versicherer ändert sich des Weiteren durch das Car- und Ridesharing als immer mehr aufkommende Smart Services. Damit sind zunächst Herausforderungen verbunden, weil mit den Sharing-Konzepten zum einen der Gesamtbestand zu versichernder Fahrzeuge zurückgeht und sich zum anderen die klassischen Deckungskonzepte nicht mehr ohne Weiteres anwenden lassen. Zur Fortbewegung mit dem Auto ist der Bürger nicht mehr länger vom Vorhandensein eines eigenen Fahrzeugs abhängig. Damit sinkt die Nachfrage nach dem eigenen Automobil und damit auch nach privaten Kfz-Versicherungen (Jäckel 2017). Der Nutzer eines Carsharing-Dienstes möchte lediglich die Möglichkeit einer flexiblen Mobilität in Anspruch nehmen, wird sich darüber hinaus jedoch mit dem Fahrzeug - das er nur für kurze Zeit nutzt - nicht weiter beschäftigen. Wichtig sind ihm lediglich die Sicherheit, der Komfort und die Effizienz der Mobilität, also die Erhältlichkeit und Funktionstüchtigkeit des Fahrzeugs, die eigene Fahrsicherheit sowie die Absicherung, falls doch etwas passiert ... und das Ganze zu einem günstigen Preis.

Der Versicherungsschutz muss in diesem Paket bereits enthalten sein, und der Nutzer möchte sich für seine flexibel und fallweise gewählten Fahrten nicht damit auseinandersetzen. Ein naheliegendes Szenario ist also, dass die Fahrzeuge künftig viel häufiger von einem OEM oder Mobilitätsanbieter und nicht vom Bürger selbst versichert werden. Die private Kfz-Versicherung dürfte damit auf Sicht immer mehr durch Policen für Fahrzeugflotten und Poolfahrzeuge abgelöst werden (Thiele und Schmidt-Jochmann 2015, S. 29). Damit verändert sich nicht nur die Tarifierung, sondern auch der Zugang zum Endkunden geht für den Versicherer zunehmend verloren. Einen noch größeren Einfluss auf die Kfz-Versicherung nimmt das autonome Fahren. Es ist zu erwarten, dass das Unfallrisiko mit autonomen Fahrzeugen erheblich sinkt und einzelne Fahrzeugversicherungen für Privatpersonen und vermutlich auch Flottenversicherungen für Geschäftskunden entbehrlich werden könnten. Ohne einen Fahrzeugführer - und wenn das Fahrzeug noch nicht einmal mehr ein Lenkrad aufweist, über das in die Steuerung eingegriffen werden könnte - wird dem Nutzer des Fahrzeugs auch kein Verschulden mehr für einen Unfall zugewiesen werden können und wird folglich auch kein individueller Versicherungsschutz mehr für den Fahrzeuglenker erforderlich sein. Die Verschiebung der Risikosituation liegt damit auf der Hand: Die Eintrittswahrscheinlichkeit eines Schadens sinkt, dafür erhöhen sich die Schadensummen angesichts der eingesetzten Technologie und möglicher Kumulsituationen. Für Unfälle kann kein Fahrer mehr, sondern muss der Hersteller oder müssen Komponentenlieferanten haftbar gemacht werden. 
Der Fokus verschiebt sich damit von der Absicherung menschlicher Fehler auf die Absicherung technischer Ausfälle oder Manipulationen. Bereits kurz- bis mittelfristig wird die fortschreitende Entwicklung der autonomen Fortbewegung das Portfolio der Versicherer weiter verändern. Neuere, zunehmend digital vernetzte Kraftfahrzeuge mit vermehrten Assistenzfunktionen bis hin zu weitgehender oder vollkommener Autonomie haben im Gegensatz zu älteren, noch im Großen und Ganzen analogen, Fahrzeugen eine geringere Unfallwahrscheinlichkeit, und die Insassen sind besser geschützt - mit allen Konsequenzen für die Begleitung der Veränderungen durch die Versicherungswirtschaft mit Korrelationsforschungen und der Entwicklung passender Deckungskonzepte und Tarifierungssysteme.

Ein weiterer, besonders wichtiger Trend in der Lebenswelt Mobilität ist die Intermodalität, also die Kombination verschiedener Verkehrsmittel. Zwar kommen die einzelnen Verkehrsmittel nach wie vor typischerweise von verschiedenen Anbietern - neue Mobilitätsformen/-angebote oftmals sogar von ganz neuen Unternehmen, wie Busanbietern (z. B. Flixbus) oder Plattformbetreibern für Car- und Ridesharing, es lassen sich aber zunehmend Konsolidierungen beobachten. Automobilkonzerne entwickeln eigene Sharing-Dienste und kaufen Mobilitäts-Start-ups auf. So hat z. B. Daimler mittlerweile u. a. den Mitfahr-Pionier flinc, das TaxiStart-up myTaxi und auch den Carsharing-Dienst car2go übernommen. Die Zahl der relevanten Marktakteure scheint also zu sinken, und dem Kunden stehen immer mehr Angebote aus einer Hand zur Verfügung.

Für den Bürger entstehen damit Vorteile: mit der moovel ${ }^{13}$-App können verschiedene Verkehrsmittel für eine bestimmte Route ausgewählt, reserviert, gebucht sowie bezahlt werden und die urbane Mobilität wird für ihre Nutzer vereinfacht. Aufgrund der großen Bedeutung einer einfachen und flexiblen Kombinierbarkeit einzelner Angebote ist gerade in der Lebenswelt Mobilität die Rolle des Orchestrators von herausragender Bedeutung und als Voraussetzung für die intermodale Mobilität anzusehen. Diese Rolle wird von Versicherern schwer einzunehmen sein, da sie im Vergleich zu den Automobilherstellern über eine geringere Größe, weniger Ressourcen - inkl. des Pools an smarten Daten - und meist einen deutlich schlechteren Zugang zum Kunden verfügen. Es muss daher eher eine andere Rolle gefunden werden, sich in dieser Lebenswelt zu positionieren.

Im Zusammenhang mit der Intermodalität könnte das Angebot einer Mobilitätsversicherung einen Lösungsansatz bieten. Die Versicherungspolice könnte

${ }^{13} 100 \%$ ige Tochter der Daimler AG. 
sich damit an ein Ökosystem der vernetzten Mobilität andocken und neben Schäden bei der Nutzung einzelner Fortbewegungsmittel auch die Nichtverfügbarkeit eines Carsharing-Fahrzeugs, die Verspätung eines öffentlichen Verkehrsmittels oder den Ausfall von weitergehenden Services bzw. Mehrwertdiensten abdecken, die rund um die Mobilität von den Anbietern versprochen wurden. Mit der Absicherung von Kfz-Schäden allein wird sich im Rahmen vernetzter Fahrzeuge nicht mehr lange ein Alleinstellungsmerkmal aufrechterhalten lassen. Durch die immer seltener werdenden Schäden, die sich immer besser prognostizieren lassen, ist damit zu rechnen, dass die verbleibenden Schäden künftig auch. von den Automobilherstellern selbst übernommen werden (wie beispielsweise bei Tesla). Eine Neuausrichtung ist für die Versicherer daher unumgänglich.

\subsection{Utopien und Dystopien}

\subsubsection{Utopien: Möglichkeiten und gesellschaftlicher Nutzen}

Die Beschreibung der Smart Services und die Analyse bestehender Geschäftsmodelle in den Lebenswelten Wohnen, Gesundheit und Mobilität haben gezeigt, dass die Analysen von Daten - auch von personenbezogenen Daten - für die Entwicklung neuer, umfassender Mehrwerte für die Bürger genutzt werden können. Die Sicherheit der Menschen kann durch smarte Lösungen erhöht, der Komfort kann gesteigert und Ressourcen können effizienter eingesetzt werden.

Schon heute besteht z. B. die Möglichkeit, einen Großteil der Wohnumgebung so zu steuern, dass alltägliche Arbeit und Aufgaben des Bewohners entfallen und durch datenbasierte Systeme übernommen werden können. Durch den Einsatz smarter Sensoren können Risiken wie Feuer (smarte Rauchmelder) und Feuchtigkeit (Leckage-Sensoren) nicht nur frühzeitig erkannt werden, vielmehr können sensorgesteuert auch präventive Maßnahmen ergriffen und Schäden von vornherein verhindert werden. Ergänzend können z. B. smarte Thermostate und Lichtsysteme die Wohnumgebung per App oder Sprachsteuerung auf die individuellen Bedürfnisse des Bewohners anpassen und damit den Lebenskomfort erhöhen und die Energieeffizienz steigern.

Doch gerade in der Lebenswelt Wohnen stecken die Smart Services noch in den Kinderschuhen; vieles ist zwar theoretisch schon möglich, kommt aber gerade in Deutschland - bislang kaum zum Einsatz. Die Gründe dafür sind vielfältig und reichen von einer teils noch komplizierten Handhabung (Stichworte: Installation und Konnektivität) bis hin zu Sicherheits- und Datenschutzbedenken 
der potenziellen Nutzer. Während aktuell noch ein gewisser Grad an technischer Versiertheit als Grundvoraussetzung für die Nutzung von Smart Home-Technologien gilt, die eher bei den jüngeren ,Digital Natives' anzutreffen ist (die vielfach noch gar keine eigene Wohnung besitzen), adressieren die Angebote künftig mehr und mehr auch Familien und ältere Menschen, die ohne nennenswerte Technologieaffinität und mittels einer immer intuitiveren Bedienbarkeit die Nutzenpotenziale der Smart Home-Lösungen erkennen. Durch smarte Assistenten wie Amazon Alexa oder Lingxi Voice Assistant ${ }^{14}$ können die Funktionen auch allein über die Sprache gesteuert werden. Der nächste Entwicklungsschritt nach der Sprachsteuerung ist die Gestensteuerung. Statt Alexa zu bitten, die Musik etwas leiser zu stellen, reichen dann einfache Handbewegungen, mit der die Lautstärke reguliert (wie bereits bei den AirPods, den Bluetooth-Kopfhörern von Apple, möglich), die Tür geöffnet oder das Rollo nach unten gefahren werden können.

Die Kombination von Touchscreens, Sprach- und Gestensteuerung in allen Lebenswelten stellt fast den Höhepunkt an Komfort für die Nutzer dar, die nur noch durch direkte Gedankensteuerung übertroffen werden kann (was inzwischen als Ergebnis neurologischer Forschungen auch nicht mehr unmöglich scheint). Und in der Lebenswelt Gesundheit entstehen dadurch Potenziale für eine effiziente computergesteuerte Unterstützung und Versorgung eingeschränkter Menschen z. B. mittels Roboter und Künstlicher Intelligenz. Noch weitergehend ist gar an die heute noch utopisch anmutende Möglichkeit zu denken, querschnittsgelähmten Menschen über Gehirnsignale, die unter Überbrückung der geschädigten Wirbelsäule über einen externen Sensor an die Nerven in den Beinen weitergeleitet werden, wieder zum Gehen zu verhelfen. Voraussetzung dafür sind weitere Forschungen über die Zusammenhänge von Gedanken und Gehirnströmen, die in datengesteuerte Systeme zu übersetzen sind.

Gerade die Skepsis, die der Technologie entgegengebracht wird, hemmt aber die Entstehung umfassender Mehrwerte und den gesellschaftlichen Nutzen. Datenanalyse und Künstliche Intelligenz sind auf Big Data angewiesen. Bspw. können in der Lebenswelt Wohnen zwar die individuellen Gewohnheiten und Routinen eines Nutzers analysiert werden, und das smarte Home kann sich auf die Bewohner einstellen. Allgemeingültige Regeln können bisher jedoch nur thesenbasiert aufgestellt und nicht vom System selbst erlernt werden, da die Datenbasis zu gering ist. Wäre jedes Haus ein Smart Home und jedes Auto-

\footnotetext{
${ }^{14}$ Chinas Pendant zu Amazon Alexa.
} 
mobil ein Connected Car, könnten alle Wohnungen, Gebäude, Fahrzeuge sowie ganze Städte und Infrastrukturen miteinander kommunizieren, Daten kombiniert und ausgewertet werden. Mit immer mehr Datenquellen entstünden immer mehr nutzenstiftende Anwendungspotenziale. Sogenannte Smart Cities verknüpfen alle in der vernetzten Stadt vorhandenen Daten. Luft-, Wasser- und Energieverbrauch der verschiedenen Gebäude, Bewegungsdaten der in der Stadt befindlichen Menschen und Informationen über die Verkehrssituation werden zentral zusammengefasst und ausgewertet. Dadurch wird eine gesamthafte Analyse der Verhältnisse in der smarten Stadt ermöglicht, zahlreiche Risiken werden erkannt, Verkehrsflüsse optimiert und der Energieverbrauch maximal effizient gestaltet. Schon heute gibt es in China solche Smart Cities (Weltspiegel 2018), in denen vielerlei Daten zusammengeführt und ausgewertet werden. Dabei ist eine Ausweitung der Nutzung über Zwecke der Stadtverwaltung hinaus für geschäftliche und auch soziale Zwecke denkbar. So können akute Engpässe oder Hilfebedarfe frühzeitig erkannt und gedeckt werden - z. B. der Bedarf nach zusätzlicher (Mobilitäts-) Kapazität bei hoher Auslastungsrate der Fahrzeuge in einem bestimmten Gebiet oder nach zusätzlicher ärztlicher (Gesundheits-)Versorgung im Fall einer beginnenden Pandemie.

Was die Mobilität angeht, wird nach dem autonomen Fahren das autonome Fliegen den nächsten Entwicklungsschritt darstellen. Autonom fliegende Drohnen können z. B. die medizinische Versorgung in ländlichen Gebieten sicherstellen, und zwar mit datenbasierten Echtzeitanalysen ebenfalls auch in Akutsituationen. Dasselbe gilt für weitere Arten von Rettungseinsätzen, z. B. bei der Feuerbekämpfung oder Lawinenbergung, wenn Sensoren selbsttätig autonome Fluggeräte (Löschflugzeuge oder Bergungsdrohnen mit Robotics-Ausstattung) anfordern und effizient steuern. Auch die mobile Alltagssicherheit kann durch eine intelligente datenbasierte Steuerung erheblich gesteigert werden. Eine smarte Verkehrsüberwachung und -lenkung verhilft dazu, Ordnungswidrigkeiten aufzudecken bzw. präventiv zu vermeiden, Verkehrsflüsse zu optimieren und Unfallzahlen massiv zu senken. Straftaten können mithilfe der Gesichtserkennung und Abgleich mit einem großen Datensatz schnell aufgeklärt und mit Aussicht darauf ebenfalls von vornherein vermieden werden. Die Polizei kann dorthin geschickt werden, wo das Risiko von Übergriffen oder öffentlicher Unruhen nach aktueller Datenlage am größten ist. Dies alles führt nicht nur im Bereich Mobilität/Verkehr zu mehr Sicherheit, sondern im gesamten öffentlichen Leben.

Zudem führt die umfassende Datenerhebung in allen Lebenswelten der Bürger zu einem umfassenderen Verständnis individueller Wünsche und Bedürfnisse. Damit können Produkte, Dienstleistungen und Prozesse optimiert und auf die jeweiligen Bedürfnisse ausgerichtet werden. Im smarten Supermarkt (online oder 
offline) werden dem Kunden nur noch Produkte angeboten bzw. hervorgehoben angezeigt, die für ihn relevant sind. Tiernahrung wird nur Tierbesitzern präsentiert, Vegetarier erhalten nur fleischlose Rezeptvorschläge und gegenüber Allergikern werden die für sie ungeeigneten Produkte markiert. Denkbar ist auch ein digitaler Assistent, der die Finanzen und Versicherungsbedarfe überwacht, analysiert und sich selbstständig um die individuell besten Lösungen kümmert.

Dies alles beschreibt eine Welt, in der sich die Bürger sicherer fühlen können, in der sie von Alltagsaufgaben entlastet sind, viele Annehmlichkeiten den Lebenskomfort erhöhen und dabei ressourceneffizient noch Zeit und Geld gespart werden können. Alles in allem ist die Utopie ein merklich erhöhtes Maß an Freiheit der Menschen, ihr Leben nach den eigenen Vorstellungen und Wünschen zu gestalten.

\subsubsection{Dystopien: Grenzen und gesellschaftliche Risiken}

Bei allen Utopien über Nutzenpotenziale, die Big Data, Data Analytics und die künstliche Intelligenz mit sich bringen, stellt sich jedoch die Frage, wer über den Einsatz der Daten entscheidet und wer damit auch bestimmt, was damit angefangen wird. Je nachdem ergibt sich auch ein Spannungsfeld, ob mit den Anwendungen eine Verbesserung oder möglicherweise auch eine Verschlechterung der Lebenssituation von Bürgern in einer Gesellschaft verbunden ist.

Als Ausgangsfrage ist zunächst zu erörtern, wem die Daten über Verhaltensweisen und Vorlieben der Menschen gehören. Die Diskussion darüber zieht sich bereits heute durch Politik, Gesellschaft und sämtliche Branchen. Einigkeit herrscht oft darüber, dass die Datenhoheit bei den Bürgern selbst liegen sollte, deren Verhältnisse und Verhaltensweisen damit repräsentiert werden. Das heißt, die Bürger selbst sollen individuell entscheiden und steuern können, welche Daten wem und für welche Zweck preisgegeben werden. Doch was bedeutet das konkret? Und steht diese Anforderung nicht gerade im Widerspruch zu den Potenzialen einer umfassenden, zunächst noch nicht zweckgebundenen Auswertung durch Behörden und Anbieter verschiedener Smart Services, um neue Anwendungsfelder überhaupt erst zu identifizieren?

Der potenzielle Nutzen einer Datenanalyse wird naturgemäß umso größer, je mehr Daten (Big Data) miteinander verknüpft und kombiniert ausgewertet werden. Voraussetzung dafür ist wiederum, dass die Daten möglichst an einer Stelle zusammenlaufen und gepoolt werden. Dadurch bilden sich jedoch Monopolisten, bei denen die Daten nicht nur erfasst, gespeichert und ausgewertet werden, 
sondern die auch die Einsatzfelder kontrollieren können. Mit der Kontrolle aller möglichen Daten geht auch die Kontrolle über die Bürger selbst einher. Von daher ist die Frage zu stellen, wem die Datenverantwortung obliegen darf und ob und inwiefern Kontrollmechanismen etabliert werden können, um den Missbrauch der Daten zu verhindern. In breiten Kreisen der Bevölkerung herrschen inzwischen erhebliche Skepsis, ein steigendes Unwohlsein und sich verstärkende Kontrollängste vor der zunehmenden Macht der großen amerikanischen Datengiganten, wie insbesondere Amazon, Apple, Facebook und Google.

Beispielsweise gewinnt Amazon durch zahlreiche Schnittstellen zwischen Alexa und Hausgeräten, Smartwatches sowie Überwachungssystemen aller Art Informationen über persönliche Aktivitäten, Gewohnheiten und intime Bedürfnisse, die zusammenlaufen und ausgewertet werden können. Mit vielen weiteren Smart Services erhält Amazon schon heute Zugang zu verschiedensten Informationen: Durch Amazon Video oder Amazon Music kennt das Unternehmen den Musik- und Filmgeschmack seiner Nutzer, durch Amazon Marketplace das Einkaufsverhalten, durch Amazon Pay das Zahlungsverhalten und die Bonität und dank Amazon Fresh und Amazon Go sind die Essgewohnheiten des Nutzers bekannt. Daneben liefern Amazon Dash ${ }^{15}$, Amazon Smile ${ }^{16}$, Amazon Publishing ${ }^{17}$, der Amazon App Store und viele weitere Amazon-Services wertvolle Informationen über die Interessen, das Konsumverhalten und die Bedürfnisse der Nutzer, die durch Kombination ein umfassendes Persönlichkeitsprofil des Bürgers ergeben. Dadurch kann Amazon jedem Nutzer die individuell passenden Produkte empfehlen und mit dem eigenen Kurierdienst (Amazon Logistik) in die Amazon Packstation oder dank Amazon Key direkt in dessen Wohnung bringen. Heute wählt der Kunde diese Produkte und den Anbieter noch selbst aus und startet nach eigenem Wunsch den Bestellvorgang. Weitergedacht entstehen durch die Datenmonopolisierung und die Perfektionierung von individuellen Offerten auch Monopole von Anbietern. Und wenn der Anbieter mithilfe künstlicher Intelligenz womöglich noch vor dem Nutzer selbst weiß, welche Produkte dieser braucht

\footnotetext{
${ }^{15}$ Mit dem Amazon Dash Button können Amazon Prime Kunden verschiedene Produkte per Knopfdruck bestellen. Dabei wird der mit WLAN verbunden Knopf bspw. an die Waschmaschine angebracht; nach dem Drücken des Knopfes wird innerhalb von $24 \mathrm{~h}$ neues Waschmittel geliefert.

${ }^{16}$ Bei Amazon Smile wählen Kunden vor dem Einkauf aus einer Liste eine von Tausenden sozialen Organisationen aus und Amazon überweist 0,5\% der Kaufsumme an die Einrichtung.

${ }^{17}$ Eigener Verlag, der Nutzern auch das Verlegen eigener Texte ermöglicht.
} 
und wie die Dienstleistungen für ihn ausgestaltet sein müssen, wird letzten Endes auch die Mündigkeit des Konsumenten immer weiter eingeschränkt.

Schon heute steuert auch Google Maps das Verhalten seiner Nutzer - nicht nur durch die vorgeschlagene Reiseroute und das Angebot besonders gut passender Verkehrsmittel, sondern auch z. B. durch individuelle Vorschläge eines nah gelegenen Restaurants oder sonstige Dienstleistungen. Der Streamingdienst Netflix wertet genau aus, welche Filme sich die Nutzer zu welcher Tageszeit anschauen, welche Genres sie präferieren, an welchen Stellen abgeschaltet wird, bis hin zur konkreten Aufmerksamkeit, die der Zuschauer einzelnen Szenen schenkt. Basierend auf diesen Erkenntnissen produziert Netflix mit großem Erfolg eigene Filme und Serien, die den Kunden individuell empfohlen werden. Im Ergebnis entscheidet der Nutzer kaum noch selbst, welche Filme er sieht.

Ähnlich zu Amazon verfügt auch das chinesische Pendant Alibaba über verschiedene Smart Services, wie einen Online-Markplatz und ein eigenes Bezahlsystem (Alipay). Alibaba kombiniert dabei die Daten, die innerhalb der verschiedenen Services gewonnen werden, und generiert daraus einen Scoring-Wert. In diesem Scoring-Wert werden das Konsum- und Zahlungsverhalten des Nutzers bewertet. Parallel lassen sich Punkte sammeln, die z. B. zum Entfall der Kaution bei Anmietung eines Fahrzeugs oder einer Wohnung genutzt werden können. Darüber hinaus könn(t)en zahlreiche weitere Daten in den Scoring-Wert integriert werden: der Bildungsgrad, der Beruf, das Konsum- und Zahlungsverhalten von Familienmitgliedern, Freizeitbeschäftigungen oder die politische Gesinnung. Neben einem System von Belohnungen durch Entfall von Kautionen oder z. B. vergünstigten Kreditkonditionen ist ebenso ein Bestrafungssystem denkbar. Beispielsweise könnten Kunden von bestimmten Produkten oder Smart Services ausgeschlossen werden, wenn sie für das Unternehmen einen bestimmten Mindestumsatz nicht überschreiten oder Kerndienste nicht in Anspruch nehmen.

Mit der Macht der Anbieter entsteht die Gefahr, dass die Nutzer manipuliert und selbstbestimmtes Verhalten beschnitten werden. Diese Gefahr steigt mit der immer größeren Bereitschaft breiter Bevölkerungskreise, trotz aller Datenschutzskepsis persönliche Daten freizugeben, um günstigere Angebote oder mehr Komfort zu erhalten, und mit der Tendenz zu oligopolistischen Datenpools oder sogar eines Datenmonopols. Schon heute verfügen nur wenige Unternehmen nahezu monopolartig über äußerst große Datensätze. Diese Konzentration der Daten bei wenigen Unternehmen resultiert in einer gewaltigen Macht, die zu einer Verletzung der Persönlichkeitsrechte der Bürger, einem Verlust von Freiheit, Demokratie und sozialen Bindungen führen könnte. 
Nicht nur Wirtschaftsunternehmen, sondern auch Behörden und staatliche Einrichtungen können die Daten und Informationen über ihre Bürger missbrauchen. Die Sozialkreditberichte, wie sie aktuell in China bereits getestet werden, sind ein Beispiel dafür. Hier ist es der Staat, der Daten sammelt, wer sich wohin bewegt hat, mit welchem Verkehrsmittel, wer dabei zu schnell gefahren ist oder bei Rot die Straße überquert hat. Übersetzt wird das ebenfalls in einen Punktestand (,Social Scoring'), der bei der Vergabe von Jobs herangezogen wird, die Festlegung von Kreditkonditionen bestimmen und auf Dating-Plattformen angegeben werden kann. Zudem kommen Konzepte in Betracht, die Bürger bei unerwünschtem Verhalten zu bestrafen, indem ihnen z. B. der Kauf von Luxusgütern oder Auslandsaufenthalte verboten werden. Aber wie erfolgt die Bewertung der Daten und damit die Übersetzung in ein Punktesystem? Während dies bei Verkehrsverstößen noch relativ objektiv möglich ist, wird es schwierig, wenn auch andere Datenquellen herangezogen und ausgewertet werden z. B. wer wie lange das Licht anhat, wer sich mit wem getroffen, welche Filme geschaut, welche Produkte gekauft, an welchen Veranstaltungen teilgenommen oder welche Partei gewählt hat. Auch daran könnten wieder Belohnungs- aber auch Bestrafungssysteme geknüpft werden. So könnten z. B. das Anmieten einer Wohnung, der Zugang zu Verkehrsmitteln, bestimmte Sozialleistungen usw. eingeschränkt oder gänzlich unterbunden werden.

Die informationelle Überwachung und digitale Bevormundung können im Zuge von Big Data und Data Analytics zu einer starken Einflussnahme auf die Gesellschaft und zu einer Verschiebung ihrer Strukturen führen (Bendel 2018b). Es drohen der ,Gläserne Bürger', d. h. der Verlust von Privatsphäre und die. Manipulation von Denken und Handeln der Menschen mittels gezielter Anreiz- und Bestrafungsmechanismen. Alles in allem ist die Dystopie ein merklich eingeschränktes Maß an Freiheit. der Menschen, ihr Leben nach den eigenen Vorstellungen und Wünschen zu gestalten. ${ }^{18}$

\footnotetext{
${ }^{18}$ Daneben besteht bei jedem vernetzten und datenbasierten System das Risiko von Fehlern, Hackerangriffen und Datendiebstahl. So wurde z. B. in China ein auf einem Bus angebrachtes Werbegesicht vom System als Fußgänger wahrgenommen, und die betreffende Person wurde in der Folge beschuldigt, eine rote Fußgängerampel überquert zu haben. Die Konsequenz war eine Aufforderung zur Strafzahlung. Was in diesem Fall eher humorvoll zu nehmen ist und ohne schwerwiegende Folgen blieb, kann grundsätzlich für den Bürger gravierende Auswirkungen haben, wenn datenbasierte Systeme auf Basis nicht mehr nachvollziehbarer Algorithmen automatisierte Folgehandlungen einleiten. Darüber hinaus können Hackerangriffe einzelne Anwendungen oder ganze Systeme lahmlegen.
} 


\section{Literatur}

Acatech. (2018). Smart Service Welt. Internetbasierte Dienste für die Wirtschaft. https:// www.acatech.de/Projekt/smart-service-welt/. Zugegriffen: 19. Dez. 2018.

ADAC. (2017). Die Evolution der Mobilität. https://www.zukunftsinstitut.de/fileadmin/ user_upload/Publikationen/Auftragsstudien/ADAC_Mobilitaet2040_Zukunftsinstitut. pdf. Zugegriffen: 11. Okt. 2018.

ADAC. (2018). Carsharing. https://www.adac.de/_mmm/pdf/fi_carsharing_sp_58306.pdf. Zugegriffen: 19. Dez. 2018.

Ahlemann, D., Koster, A., Crusius, D., \& Kerstan, H. (2016). Growth in a commoditizing market. In R. Vierreckl, A. Koster, E. Hirsh, \& D. Ahlemann (Hrsg.), Connected car report 2016: Opportunities, risk, and turmoil on the road to autonomous vehicles (S. 23-30). https://www.strategyand.pwc.com/media/file/Connected-car-report-2016. pdf. Zugegriffen: 19. Dez. 2018.

Akoto, P. (2018). Studie bemängelt Datenschutz bei Smart-Home-Technik. https://www. energate-messenger.de/news/183318/-studie-bemaengelt-datenschutz-bei-smart-home-technik. Zugegriffen: 19. Dez. 2018.

Amazon. (2018). Amazon key smart lock kit. https://www.amazon.com/ $\mathrm{b}$ ?ie $=\mathrm{UTF} 8 \&$ node $=1$. Zugegriffen: 10. Okt. 2018.

Ambient Assisted Living Deutschland. Technik die unser Leben vereinfacht. http://www. aal-deutschland.de/. Zugegriffen: 11. Okt. 2018.

Apple. (2018a). Die Health-App auf Ihrem iPhone oder iPod touch verwenden. https:// www.apple.com/de/apple-watch-series-4/health/. Zugegriffen: 11. Okt. 2018.

Apple. (2018b). Gesundheit \& Fitness. https://www.apple.com/de/shop/iphone/iphone-accessories/health-fitness. Zugegriffen: 13. Dez. 2018.

Apple. (2018c). Mehr Möglichkeiten für Forschung, Ärzte und dich. https://www.apple. com/de/researchkit/. Zugegriffen: 11. Dez. 2018.

Apple. (2018d). So hast du noch nie auf deine Gesundheit geachtet. https://www.apple. com/de/ios/health/. Zugegriffen: 13. Dez. 2018.

Apple. (2018e). Doctors put patients in charge with Apple's health records feature. https:// www.apple.com/newsroom/2018/03/doctors-put-patients-in-charge-with-apples-healthrecords-feature/. Zugegriffen: 13. Dez. 2018.

Arbeitskreis Smart Services Welt. (2015). Smart Service Welt: Umsetzungsempfehlungen für das Zukunftsprojekt internetbasierte Dienste für die Wirtschaft. https://www.acatech.de/wp-content/uploads/2015/03/BerichtSmartService2015_mitUmschlag_bf.pdf. Zugegriffen: 19. Dez. 2018.

Während in einem solchen Fall bspw. die Manipulation von Thermostaten in der Lebenswelt Wohnen nur zu einem Ausfall der Heizungen führt, können etwa in der Lebenswelt Mobilität autonome Fahrzeuge oder in der Lebenswelt Gesundheit medizinische Geräte fehlgesteuert werden, was unmittelbar Menschenleben gefährdet. Solcherart technische Risiken automatisierter datengetriebener Systeme waren allerdings nicht im Fokus der vorliegenden Studie. 
Ärztezeitung. (2017). AGES. Online-Umfrage zu Gesundheitsrisiken. http://www.aerztezeitung.at/archiv/oeaez-2017/oeaez-3-10022017/elga-datenmissbrauch-hacker-gesundheitsdaten.html. Zugegriffen: 13. Dez. 2018.

Bardmann, M. (2019). Grundlagen der Allgemeinen Betriebswirtschaftslehre. Geschichte Konzepte - Digitalisierung. Wiesbaden, Springer Fachmedien.

Bass J. (2017): Chancen der Digitalisierung aus Sicht der Krankenkassen, in: DigitalGipfel Plattform Innovative Digitalisierung der Wirtschaft Fokusgruppe Intelligente Vernetzung (Hrsg.): Deutschland intelligent vernetzt. Digitale Gesundheit 2017. DIV Report Spezial, Juni 2017, S. 33-34. https://deutschland-intelligent-vernetzt.org/app/ uploads/2017/06/DIV_Report_Spezial_Gesundheit2017.pdf

Becker, D., \& Pawelke, M. (2015). Automotive. Blechbieger oder Grid Master? Die Automobilindustrie an der Weggabelung in ein hochdigitalisiertes Zeitalter. https://assets. kpmg/content/dam/kpmg/pdf/2015/11/blechbieger-oder-grid-master.PDF. Zugegriffen: 13. Dez. 2018.

Beenken, M., Knörrer, D., Moormann, J., \& Schmidt, D. (Hrsg.). (2018). Digital Insurance: Strategien, Geschäftsmodelle, Daten. Frankfurt a. M.: Frankfurt School.

Bendel, O. (2018a). Smart home. https://wirtschaftslexikon.gabler.de/definition/smarthome-54137. Zugegriffen: 10. Okt. 2018.

Bendel, O. (2018b). Big data. https://wirtschaftslexikon.gabler.de/definition/bigdata-54101. Zugegriffen: 11. Dez. 2018.

Bratzel, M., \& Tellermann, R. (2018). Finanzierung und Absicherung neuer Mobilitätskonzepte. https://auto-institut.de/index_htm_files/Finanzierung_und_Absicherung_ neuer_Mobilitaetskonzepte.pdf. Zugegriffen: 10. Okt. 2018.

Bundesinstitut für Bevölkerungsforschung. (1996). Bundesinstitut für Bevölkerungsforschung. Die Lebenssituation älterer Menschen. Expertise für die Kölnische Rückversicherungsgesellschaft. Köln: $\mathrm{o}$. V.

Bundesministerium für Wirtschaft und Energie. (2014). Entwicklung digitaler Technologien. Die Zukunft der Wirtschaft ist digital. https://www.bmwi.de/Redaktion/DE/ Publikationen/Technologie/entwicklung-konvergenter-ikt.pdf?_blob=publicationFile\&v=5. Zugegriffen: 19. Dez. 2018.

Bundesministerium für Wirtschaft und Energie (2017a). Smart Service Welt- Internetbasierte Dienste für die Wirtschaft. https://www.bmwi.de/Redaktion/DE/Publikationen/ Digitale-Welt/smart-service-welt-internetbasierte-dienste-fuer-die-wirtschaft.pdf? blob $=$ publicationFile \&v=9. Zugegriffen: 19. Dez. 2018 .

Bundesministerium für Wirtschaft und Energie. (2017b). Digitale Geschäftsmodelle. https://www.bmwi.de/Redaktion/DE/Publikationen/Mittelstand/mittelstand-digital-digitale-geschaeftsmodelle.pdf?_blob=publicationFile\&v=15. Zugegriffen: 19. Dez. 2018.

Bundesverband Digitale Wirtschaft. (2018a). Wie smart ist das Recht im Smart Home. https://www.bvdw.org/fileadmin/bvdw/upload/publikationen/RZ_BVDW_LF_Recht_ im_SmartHome_web_20180611.pdf. Zugegriffen: 10. Okt. 2018.

Bundesverband Digitale Wirtschaft. (2018b). Wem „gehören“ die Daten im Connected Car? https://www.bvdw.org/fileadmin/bvdw/upload/publikationen/connected_mobility/ BVDW_Daten-im-Connected-Car_final.pdf. Zugegriffen: 10. Okt. 2018. 
Business Panorama. (2017). UBTECH Robotics präsentiert Lynx, den interaktiven humanoiden Roboter mit Amazon Alexa. http://business-panorama.de/news.php?newsid=404118. Zugegriffen: 11. Dez. 2018.

Chaudhuri, A. (2018). Predictive maintenance for industrial iot of vehicle fleets using hierarchical modified fuzzy support vector machine. https://arxiv.org/ftp/arxiv/ papers/1806/1806.09612.pdf. Zugegriffen: 19. Dez. 2018.

Cloverhealth. (2018). Clover is a medicare plan done differently. https://www.cloverhealth. com/en/plans. Zugegriffen: 13. Dez. 2018.

Connected Living. (2018). Unsere Mitglieder. https://connected-living.org/organisation. Zugegriffen: 19. Dez. 2018.

Corthier, J., Kreuter, V., \& Klingholz, R. (2011). Lebenswelten 2015. Wie sich die Struktur der Konsumenten in den Regionen verändert. Nürnberg: GfK.

Deloitte. (2018). Smart Home Consumer Survey 2018. Ausgewählte Ergebnisse für den deutschen Markt. https://www2.deloitte.com/content/dam/Deloitte/de/Documents/ technology-media-telecommunications/Deloitte_TMT_Smart_Home_Studie_18.pdf. Zugegriffen: 17. Dez. 2018.

Denkena, B., Dittrich, M.-A., Uhlich, F., Maibaum, L., \& Mörke, T. (2017). Das gentelligente Werkstück. In G. Reinhart (Hrsg.), Handbuch Industrie 4.0: Geschäftsmodelle, Prozesse, Technik (S. 239-321). München: Hanser.

Deutsche Gesellschaft für Qualität. (2017). Datenschutz und die EU-DSGVO im Gesundheitswesen. http://blog.dgq.de/datenschutz-und-die-eu-dsgvo-im-gesundheitswesen/. Zugegriffen: 13. Dez. 2018.

Deutsches Institut für Vertrauen und Sicherheit im Internet. (2016). Big data. https://www. divsi.de/wp-content/uploads/2016/01/Big-Data.pdf. Zugegriffen: 19. Dez. 2018.

Discovergy. (2018). Discovering the Smart Home. Mit Discovergy und iHaus. https://discovergy.com/smart-home-ihaus. Zugegriffen: 17. Dez. 2018.

Duden. (2019). Lebenswelt. https://www.duden.de/suchen/dudenonline/lebenswelt. Zugegriffen: 10. Okt. 2018.

Eckstein, L., Form, T., \& Maurer, M. (2018). Automatisiertes Fahren, VDI-Statusreport Juli 2018. https://www.trialog-publishers.de/media-online/automatisiertes-Fahren-VDIStatusreport-Juli-2018.pdf.

Fasel, D., \& Meier, A. (Hrsg.). (2016). Big Data - Grundlagen, Systeme und Nutzungspotenziale. Wiesbaden: Springer Vieweg.

Gesetz zur Bevorrechtigung des Carsharing. (2017) vom 05.07.2018 (BGBI. I S. 2230) in der Fassung der Bekanntmachung vom 05.07.2018 (BGBI. I S. 2230). https:// www.bgbl.de/xaver/bgbl/start.xav?startbk=Bundesanzeiger_BGB1\&start=//*\%5b@ attr_id=\%27bgbl117s2230.pdf\%27\%5d\#__bgbl__\%2F\%2F*\%5B\%40attr_ id\%3D\%27bgbl117s2230.pdf\%27\%5D_1555779424306. Zugegriffen: 19. Dez. 2018.

Goodwall, W., Fishman, T. D., Bornstein, J., \& Bronthron, B. (2017). The rise of mobility as a service. Deloitte Review, 20, 111-129.

Grohe. (2018). Smarte Kooperation: Grohe und Qivicon lassen Wasserschäden im intelligenten Zuhause keine Chance. https://www.grohe.com/de/corporate/news/category_ news/pressreleases/pressrelease/news_10368.html. Zugegriffen: 18. Dez. 2018.

Grösser, S. (2018). Geschäftsmodell. https://wirtschaftslexikon.gabler.de/definition/geschaeftsmodell-52275. Zugegriffen: 10. Okt. 2018. 
Gründinger, W. (2018). Smart Mobility Initiative. Eine diskriminierungsfreie Plattform für multimodale Mobilität. https://www.bvdw.org/fileadmin/bvdw/upload/publikationen/ connected_mobility/BVDW_Smart_Mobility_Initiative_konzept-v2.pdf. Zugegriffen: 10. Okt. 2018.

Hartmut, S., Seidel, U., \& Knape, T. (2010). Smart Home in Deutschland. Institut für Innovation und Technik (iit). In VDI/VDE-IT (Hrsg.).

Heide, D. (2018). Zu langsam für die Digitalisierung. https://www.handelsblatt.com/ unternehmen/it-medien/gesetze-in-deutschland-zu-langsam-fuer-die-digitalisierung/20828056.html?ticket=ST-208995-U6cCswFE0smKwfrkpYYt-ap5. Zugegriffen: 19. Dez. 2018.

Heise online. (2017). Amazon Key. Smartes Türschloss soll Paketboten reinlassen. https:// www.heise.de/newsticker/meldung/Amazon-Key-Smartes-Tuerschloss-soll-Paketboten-reinlassen-3872147.html. Zugegriffen: 10. Okt. 2018.

Heyen, N. B. (2016). Digitale Selbstvermessung und Quantified Self. Potentiale, Risiken und Handlungsoptionen. Karlsruhe: Frauenhofer ISI.

Hioscar. (2018). Get more from your health insurance. https://www.hioscar.com/individuals/ny. Zugegriffen: 13. Dez. 2018. Hofer, a.a.O (Fn. 3).

IBM (2019). Infographics \& animations: The four V's of big data. https://www.ibmbigdatahub.com/infographic/four-vs-big-data. Zugegriffen: 19. Dez. 2018.

Ignor, S. (2018). Amazon Key. Jetzt kommen die Pakete direkt ins Auto. https://www. computerbild.de/artikel/cb-News-Vernetztes-Wohnen-Amazon-Key-PaketboteWohnung-Auto-19247545.html. Zugegriffen: 10. Okt. 2018.

Industriepraxis. (2018). IFA 2017. UBTECH präsentiert Zukunft humanoider Roboter. http://industriepraxis.de/ifa-2017-ubtech-praesentiert-zukunft-humanoider-roboter/. Zugegriffen: 24. Nov. 2018.

Innogy. (2017). Geofencing mit innogy SmartHome. https://news.innogy.com/geofencing-mit-innogy-smarthome/. Zugegriffen: 17. Dez. 2018.

Jäckel, T. (2017). Sharing Economy. Mobilität der Zukunft. https://www.versicherungsbote. de/id/4860853/Sharing-Economy-Mobilitaet-der-Zukunft/. Zugegriffen: 20. Dez. 2018.

Kapalschinski, C. (2017). Amazons tückisches Türschloss. https://www.handelsblatt. com/unternehmen/handel-konsumgueter/onlinehandel-amazons-tueckisches-tuerschloss/20501516.html?ticket=ST-870154-3rzc7wvOxNLVhOFfN10i-ap5. Zugegriffen: 10. Okt. 2018.

Kraus, B. (2006). Lebenswelt und Lebensorientierung. Eine begriffliche Revision als Angebot an eine systematisch-konstruktivistische Sozialarbeitswissenschaft. Zeitschrift für systemische Therapie und Familientherapie, 37(2), 116-129.

Krohn, P. (2018). Soll die Versicherung das Haus kontrollieren? https://www.faz.net/aktuell/finanzen/meine-finanzen/versichern-und-schuetzen/smarte-versicherungen-haben-esbisher-kaum-in-die-deutschen-haeuser-geschafft-15411803.html. Zugegriffen: 19. Dez. 2018.

Lacace, M. (2017). Meet lynx, the walking, talking home robot powered by Amazon Alexa. https://www.androidcentral.com/meet-lynx-walking-talking-robot-powered-amazonalexa. Zugegriffen: 17. Dez. 2018.

Mamerow, S. (2012). Entscheiden und Wirtschaften: Eine Analyse des wirtschaftlichen Alltags unter anthropologischem Blickwinkel. Hamburg: Diplomica.

Moovel. (2018). Unsere Vision ist eine Welt ohne Staus. https://www.moovel.com/de. Zugegriffen: 19. Dez. 2018. 
Müller, R. (2018). Smart-Home-Versicherung. Sinnvoll oder nutzlos? https://www.connect. de/ratgeber/smart-home-versicherung-sinnvoll-nutzlos-3198396.html. Zugegriffen: 19. Dez. 2018.

Munich RE. (2017). Smart Home: Potenziale für die Assekuranz. https://www.munichre. com/topics-online/de/digitalisation/smart-home-potential-insurers.html. Zugegriffen: 19. Dez. 2018.

Nationaler IT-Gipfel. (2015). Smart Home. Ergebnisdokument der Projektgruppe Smart Home. https://plattform-digitale-netze.de/app/uploads/2016/06/151105_PF1_007_FG1_ Ergebnisdokument-Projektgruppe-Smart-Home.pdf. Zugegriffen: 19. Dez. 2018.

Nitschke, T. (2018). BLC Studie Datenschutz 2018. http://berg-lund.de/publikationen/ datenschutz-2018. Zugegriffen: 19. Dez. 2018.

Oliver Wyman. (2018). Mitarbeiter der Zukunft: Die Automobilindustrie muss handeln. https://www.oliverwyman.de/content/dam/oliver-wyman/v2-de/media/2018/Pressemitteilungen/Kompetenzwandel-in-der-Automobilindustrie_PM_OliverWyman.pdf. Zugegriffen: 19. Dez. 2018.

Osterwalder, A., \& Pigneur, Y. (2010). Business model generation. A handbook for visionaries, game changer, and challengers. New Jersey: Wiley.

PwC. (2017). Sherlock in health. How artificial intelligence may improve quality and efficiency, whilst reducing healthcare costs in Europe. https://www.pwc.de/de/gesundheitswesen-und-pharma/studie-sherlock-in-health.pdf. Zugegriffen: 19. Dez. 2018.

PwC. (2018). Wie Tech Giganten den Gesundheitsmarkt revolutionieren. https://www.pwc. de/de/gesundheitswesen-und-pharma/wie-tech-giganten-den-gesundheitsmarkt-revolutionieren.html. Zugegriffen: 13. Dez. 2018.

Randelhoff, M. (2014). Definition. UberPop, WunderCar, Lyft \& Co. - Ridesharing oder vielmehr Rideselling? https://www.zukunft-mobilitaet.net/74151/analyse/definition-ridesharing-rideselling-unterschiede-taxi-carpooling/. Zugegriffen: 19. Dez. 2018.

ReachNow. (2018). Where to? https://reachnow.com/en/. Zugegriffen: 19. Dez. 2018.

Rohkamm, F. T. (19.11.2018). Connected, Autonomous, Shared \& Electric: Perspektiven für die Mobilität, deren Risiken und Versicherungsdeckungen. Vortrag auf der InnoVario, Bonn.

Roussem, M. (2017). Maschinendaten. https://www.searchenterprisesoftware.de/definition/ Maschinendaten. Zugegriffen: 1. Okt. 2018.

Schaal, S. (2016). Von der Versuchsküche zum Geschäftsmodell. https://www.wiwo.de/ unternehmen/auto/carsharing-bmw-testet-premium-carsharing-in-den-usa/13450910-2. html. Zugegriffen: 19. Dez. 2018.

Schlingensiepen, I. (2017). Digitalisierung. Die meisten Patienten würden Gesundheitsdaten mit Ärzten teilen. https://www.aerztezeitung.de/praxis_wirtschaft/e-health/ article/943537/digitalisierung-meisten-patienten-wuerden-gesundheitsdaten-aerzten-teilen.html. Zugegriffen: 13. Dez. 2018.

Schmidt-Lackner, M. (2016). ReachNow. BMW macht Audi beim Premium-Carsharing Konkurrenz. https://www.automobilwoche.de/article/20160408/ NACHRICHTEN/160409917/reachnow-bmw-macht-audi-beim-premium-carsharing-konkurrenz. Zugegriffen: 19. Dez. 2018.

Schütz, A., \& Luckmann, T. (2017). Strukturen der Lebenswelt (2. Aufl.). Konstanz: UTB. 
Seiberth, G., \& Gründinger, W. (2018). Data-driven business models in connected cars, mobility services \& beyond. https://www.bvdw.org/fileadmin/user_upload/20180509_ bvdw_accenture_studie_datadrivenbusinessmodels.pdf. Zugegriffen: 19. Dez. 2018.

Siepermann, M. (2018). Ambient assisted living. https://wirtschaftslexikon.gabler.de/definition/ambient-assisted-living-53583. Zugegriffen: 17. Dez. 2018.

Siethoff, P. (2017). Amazon Key. Das bisschen Privatsphäre für ein Plus an Komfort. https://www.business-punk.com/2017/10/amazon-key-privacy/. Zugegriffen: 17. Dez. 2018.

SmartHome Initiative Deutschland und mm1 (o.J.). Smart Home- und AAL-Technologien in der Immobilien- und Wohnungswirtschaft. https://web.gdw.de/uploads/pdf/ studien_gutachten/Studie_Smart_Home_und_AAL_in_der_Wohnungswirtschaft.pdf. Zugegriffen: 8. Okt. 2018.

Sobe, P. (2018). Datenstrukturen und Speicherstrukturen. https://www2.htw-dresden. de/ sobe/Vorjahre/Vo_InfoMB_Jg11/7_Datenstrukturen.pdf. Zugegriffen: 8. Okt. 2018.

Spektrum. (2001). Lebenswelt. https://www.spektrum.de/lexikon/geographie/lebenswelt/4677. Zugegriffen: 10. Okt. 2018.

Stanford Medicine. (2018). Apple heart study. http://med.stanford.edu/appleheartstudy. html. Zugegriffen: 13. Dez. 2018.

Statista. (2019). Prognose zum Volumen der jährlich generierten digitalen Datenmenge weltweit in den Jahren 2018 und 2015 (in Zettabyte). https://de.statista.com/statistik/ daten/studie/267974/umfrage/prognose-zum-weltweit-generierten-datenvolumen/. Zugegriffen: 19. Dez. 2018.

Strom Magazin. (2018). Solaranlagen \& Smart Home. Eigenverbrauch komfortabel steigern. https://www.strom-magazin.de/ratgeber/solaranlage-smart-home-eigenverbrauch/. Zugegriffen: 17. Dez. 2018.

Tesla. (2018a). Introducing software version 9.0. https://www.tesla.com/de_DE/blog/introducing-software-version-9. Zugegriffen: 19. Dez. 2018.

Tesla. (2018b). Software-Version 9.0 entdecken. https://www.tesla.com/de_DE/support/ software-v9. Zugegriffen: 19. Dez. 2018.

Thiele, J., \& Schmidt-Jochmann, C. (2015). Geschäftsmodell der Kfz-Versicherung im Umbruch. https://www.rolandberger.com/de/Publications/Gesch\%C3\%A4ftsmodell-derKfz-Versicherung-im-Umbruch.html. Zugegriffen: 19. Dez. 2018.

Ubtech. (2018). Lynx. https://ubtrobot.com/products/lynx. Zugegriffen: 10. Okt. 2018.

Verordnung des Europäischen Parlaments und des Rates 2016/679 (2018). Verordnung (EU) 2016/679 des Europäischen Parlaments und des Rates vom 27.04.2018 zum Schutz natürlicher Personen bei der Verarbeitung personenbezogener Daten, zum freien Datenverkehr und zur Aufhebung der Richtlinie 95/46/EG. https://eur-lex.europa.eu/ legal-content/DE/TXT/?uri=CELEX\%3A32016R0679. Zugegriffen: 19. Dez. 2018.

Versicherungsbote. (2018). Verbraucher haben Interesse an Versicherungen mit SmartHome-Technik. https://www.versicherungsbote.de/id/4869097/Versicherungen-SmartHome-Technik/. Zugegriffen: 19. Dez. 2018.

Weltspiegel. (15. Dezember 2018). Unsere digitale Zukunft? China und die künstliche Intelligenz. ARD. 
Wired. (2018). China überwacht Autofahrer jetzt mit Drohnen. https://www.wired.de/ article/china-setzt-drohnen-fuer-die-verkehrsueberwachung-ein. Zugegriffen: 19. Dez. 2018.

Wollaston, V. (2017). Meet Lionbot, Lynx and Cruzr, the latest humanoids coming for homes and offices. https://www.wired.co.uk/article/ubtech-robots-ces-2017. Zugegriffen: 10. Okt. 2018.

Open Access Dieses Kapitel wird unter der Creative Commons Namensnennung 4.0 International Lizenz (http://creativecommons.org/licenses/by/4.0/deed.de) veröffentlicht, welche die Nutzung, Vervielfältigung, Bearbeitung, Verbreitung und Wiedergabe in jeglichem Medium und Format erlaubt, sofern Sie den/die ursprünglichen Autor(en) und die Quelle ordnungsgemäß nennen, einen Link zur Creative Commons Lizenz beifügen und angeben, ob Änderungen vorgenommen wurden.

Die in diesem Kapitel enthaltenen Bilder und sonstiges Drittmaterial unterliegen ebenfalls der genannten Creative Commons Lizenz, sofern sich aus der Abbildungslegende nichts anderes ergibt. Sofern das betreffende Material nicht unter der genannten Creative Commons Lizenz steht und die betreffende Handlung nicht nach gesetzlichen Vorschriften erlaubt ist, ist für die oben aufgeführten Weiterverwendungen des Materials die Einwilligung des jeweiligen Rechteinhabers einzuholen.

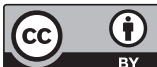

